\title{
Allgemeiner Überblick
}

Diese Statistik berichtet eingehend über 236 deutsche und 54 ausländische Unternehmungen. Von den deutschen Unternehmungen sind 107 Aktiengesellschaften; die übrigen beruhen, abgesehen von wenigen Einzelunternehmern und Genossenschaften mit beschränkter Haftpflicht, auf dem Grundsatze der Gegenseitigkeit.

Die Gegenseitigkeitsvereine beschränkten ihren Betrieb fast alle auf einen einzelnen Geschäftszweig; nur $\left.9(9)^{*}\right)$ von ihnen betätigten sich gleichzeitig in verschiedenen Versicherungszweigen; alle arbeiteten vornehmlich direkt, kein Verein betrieb lediglich indirekte Geschäfte. Von den 107 Aktiengesellschaften waren 25 (25) ausschließliche Rückversicherungsunternehmungen, sämtlich mit mehreren Zweigen; von den übrigen, auch direkt arbeitenden 82 (77) Aktiengesellschaften pflegten 47 (43) mehrere Zweige. Die kleineren Geschäftszweige: die Sturmschäden-, die Wasserleitungsschäden-, die Glas-, die Kautions-, Garantie-, Kredit- sowie die Maschinenversicherung, aber auch die Unfall- und Haftpflichtversicherung, werden fast ausschließlich von Aktiengesellschaften gepflegt.

Von den in dieser Statistik ausführlich behandelten direkt versichernden deutschen Unternehmungen haben im Berichtsjahre gearbeitet in der Lebensversicherung 28 (28) Aktiengesellschaften und 34 (35) Gegenseitigkeitsvereine, in der Unfallversicherung 27 (27) Aktiengesellschaften und 1 (1) Gegenseitigkeitsverein, in der Haftptlichtversicherung 26 (26) Aktiengesellschaften und 1 (1) Verein, in der Viehversicherung nur Gegenseitigkeitsvereine, und zwar 29 (28), in der Hagelversicherung 5 (5) Aktiengesellschaften (sämtlich mit Ausschluß anderer Versicherungsgeschäfte) und 12 (12) Gegenseitigkeitsvereine (von denen drei auch die Feuerversicherung und einer auch die Baulastversicherung betrieben), in der Feuerversicherung 36 (32) Aktiengesellschaften und 20 (20) Gegenseitigkeitsvereine, in der Versicherung gegen Einbruchdiebstahl 39 (33) Aktiengesellschaften und 4 (4) Gegenseitigkeitsvereine, und endlich in der Glasversicherung 21 (19) Aktiengesellschaften und 1 (3) Gegenseitigkeitsverein. Bei 36 (36) Kaskoversicherungsunternehmungen waren am Schlusse des Berichtsjahrs fast 9000 (8800) Fahrzeuge im Werte von 94,69 $(90,96)$ Millionen Mark versichert.

Auch die 25 (25) Rückversicherungsgesellschaften haben sich mit fast allen aufsichtspflichtigen Versicherungszweigen befaßt, und zwar (abgesehen von den weniger wichtigen Zweigen): 13 (15) mit der Lebensversicherung, 14 (14) mit der Unfallversicherung, 12 (12) mit der Haftpflichtversicherung, 2 (3) mit der Vieh- und 2 (2) mit der Hagelversicherung, 24 (24) mit der Feuerversicherung, 17 (16) mit der Einbruchdiebstahlversicherung und 7 (4) mit der Glasversicherung.

Von den 54 (55). ausländischen in dieser Statistik behandelten Unternehmungen hatten ihren Hauptsitz: 4 (4) in den Vereinigten Staaten von Amerika, 2 (3) in Belgien, 2 (2) in Dänemark, 16 (16) in England, 3 (3) in Frankreich, 4 (4) in den Niederlanden, 12 (12) in Österreich, 2 (2) in Schweden und 9 (9) in der Schweiz. Gearbeitet haben $21(21)$ in der Lebensversicherung, 8 (8) in der Unfall- und Haftpflichtversicherung, $26(27)$ in der Feuerversicherung, 14 (13) in der Einbruchdiebstahl- und 4 (6) in der Glasversicherung. Die geringfügigen Kautions- und Verun-

*) Die eingeklammerten Zahlen in diesem allgemeinen Uberblicke beziehen sich auf das vorige Jahr. 
treuungsversicherungsgeschäfte, die von 2 (2) dieser Gesellschaften abgeschlossen sind, und die Geschäfte je zweier Gesellschaften in der Wasserleitungsschäden- wie in der Automobilversicherung haben keine weitere Behandlung gefunden.

Der Umfang des Versicherungsgeschäfts der auch direkt arbeitenden deutschen Unternehmungen im Jahre 1910 wird durch die folgenden Ubersichten veranschaulicht, welche wegen der tiefgehenden Verschiedenheit der Grundlagen die Lebensversicherung einerseits und die übrigen Versicherungszweige anderseits getrennt behandeln.

\section{Umfang des Geschäfts der deutschen Versicherungsunternehmungen}

a) Lebensversicherung

\begin{tabular}{|c|c|c|c|c|c|c|c|c|}
\hline \multirow{2}{*}{ Versicherungsart } & \multirow{2}{*}{$\begin{array}{c}\text { Zahl der } \\
\text { Policen }\end{array}$} & \multirow{2}{*}{$\begin{array}{c}\text { Versiche- } \\
\text { rungs- } \\
\text { summen } \\
1000 \mathscr{M}\end{array}$} & \multicolumn{4}{|c|}{$\begin{array}{l}\text { Zunahme (Abnahme -) } \\
\text { gegen das Vorjahr }\end{array}$} & \multicolumn{2}{|c|}{$\begin{array}{c}\text { Durchschnitt- } \\
\text { licher Betrag } \\
\text { einer Police }\end{array}$} \\
\hline & & & Policen & $\%$ & $\begin{array}{l}\text { Summen } \\
1000 \mathscr{A}\end{array}$ & $\%$ & $\begin{array}{c}\text { Ende } \\
1910 \\
\mathscr{H} \\
\end{array}$ & $\begin{array}{c}\text { Ende } \\
1909 \\
\mathscr{N} \\
\end{array}$ \\
\hline Versicherung auf den Todesfall... & $228078 \mathrm{r}$ & I 1044919 & $13072 \mathrm{I}$ & 6,08 & $73^{2} 392$ & 7,10 & 4843 & 4796 \\
\hline Versicherung auf den Lebensfall .. & 498568 & 819368 & -12199 & $-2,39$ & -16751 & $-2,00$ & I 643 & I 637 \\
\hline Rentenversicherung (Jahresrenten). & 78158 & 31092 & I 584 & 2,07 & I 705 & 5.80 & 398 & 384 \\
\hline Volksversicherung $\ldots \ldots \ldots \ldots \ldots$ & 7225426 & I 360737 & 386607 & 5,65 & 89375 & 7,03 & 188 & 186 \\
\hline Zeitungsabonnentenversicherung ... & * I 166766 & $9475 \mathrm{I}$ & $*^{*} 113501$ & 10,78 & 9716 & II, 43 & 81 & $8 \mathbf{I}$ \\
\hline Sonstige kleine Versicherung... . & 727818 & $34055^{6}$ & 30877 & 4,43 & 25113 & 7,96 & 468 & 453 \\
\hline
\end{tabular}

Vergleicht man diese Zahlen mit denen des Vorjahrs, so findet man bei der Versicherung auf den Lebensfall, daß die Abnahme bei der Zahl der Policen wie bei den Versicherungssummen etwas größer geworden ist; der durchschnittlich auf' eine Police entfallende Betrag ist fortdauernd, obwohl langsam, gewachsen: von $1570 \mathscr{M} \mathrm{im}$ Jahre 1902 auf $1643 \mathscr{M}$ im Berichtsjahre. Die Ursache des fortdauernden Rückganges dieser Versicherungsart ist darin zu suchen, daß die Aussteuerversicherung jetzt vielfach durch die gemischte Versicherung und die Versicherung mit festem Auszahlungstermin, Versicherungsarten, die zu der Todesfallversicherung gerechnet werden, ersetzt wird. Wenn man also findet, daß alle anderen Versicherungsarten an Umfang zugenommen haben, so hängt das zum Teil auch mit dem Rückgange der Versicherung auf den Lebensfall zusammen. - Der Zuwachs der Volksversicherung hat in den letzten Jahren etwas nachgelassen. Bis einschließlich 1904 belief er sich bei den Policen und bei den Summen auf ungefähr 11 Prozent des Bestandes zu Anfang des Jahres; seitdem ist er bei den Summen auf 7 Prozent gesunken, bei den Policenzahlen auf etwas weniger als 5 Prozent im Jahre 1909, im Berichtsjahre hat er um ein geringes wieder zugenommen. Die durchschnittliche Versicherungssumme hat zwischen 175 und $188 \mathscr{M}$ geschwankt. - Bei der großen Todesfallversicherung ist die Zunahme der gesamten Versicherungssummen von Jahr zu Jahr ein wenig gewachsen, von 4,89 Prozent im Jahre 1903 auf 7,10 Prozent im Berichtsjahre. Die Zahl der Policen ist weniger gleichmäßig angestiegen, nämlich im Jahre 1903 um 4,54 Prozent, dann um 4,60, danach um 3,70, um 4,77, um 5,77, um 5,16, um 5,61 und im Berichtsjahr um 6,08 Prozent. Der durchschnittlich auf eine Potice kommende Betrag hat folgende Entwicke- 
lung genommen: Ende 1903 stellte er sich auf $4533 \mathscr{M}$, Ende des folgenden Jahres auf $4568 \mathscr{N}$, danach auf $4654,4702,4731,4748,4796$ und jetzt auf $4843 \mathscr{M}$. Das Rentengeschäft scheint eine weniger gleichmäßige Entwickelung zu nehmen. Der Zuwachs hat nämlich betragen in Hundertsteln des Bestandes

$\begin{array}{rrrrrr}\text { zu Anfang des Jahres } \ldots \ldots \ldots & 1906 & 1907 & 1908 & 1909 & 1910 \\ \text { bei der Policenzahl } \ldots \ldots \ldots \ldots \ldots \ldots \ldots \ldots \ldots & 3,80 & -0,02 & -0,76 & 2,03 & 2,07 \\ \text { bei dem Jahresbetrage der versicherten Renten. } & 6,16 & 2,76 & 4,06 & 5,85 & 5,80\end{array}$

Im Berichtsjahre sind von den Versicherten an Prämien und Policegebühren an die Unternehmungen gezahlt worden: in der Volksversicherung einschließlich der Zeitungsabonnentenversicherung 107,49 $(97,94)$ Millionen und in der großen Versicherung einschließlich der "sonstigen kleinen Versicherung " 511,14 (476,95) Millionen Mark; in demselben Jahre sind für eingetretene Versicherungsfälle und tür vorzeitig aufgelöste Versicherungen in der Volksversicherung ohne die mit den Summen ausgezahlten Gewinnanteile 39,06 $(36,74)$ Millionen Mark und in der großen Versicherung 2 73,26 $(\mathbf{2 6 4 , 9 0 )}$ Millionen Mark bar an die Versicherten zurückgeflossen und außerdem 34,40 $(30,39)$ Millionen Mark und 212,41 $(194,28)$ Millionen Mark, überwiegend aus den Beitragseinnahmen, den Prämienreserven zugeführt worden.

Eine Vergleichung der Änderungen des Bestandes in den letzten fünf Jahren zeigt bei einigen Versicherungszweigen recht beträchtliche Schwankungen. So hat bei der Unfallversicherung die Zahl der Policen unregelmäßig zugenommen, nämlich I 906 um 7,6, 1907 um 7,5, 1908 um 6,1, 1909 um 7,6 und 1910 um 8,6 Prozent. Die Haftpflichtversicherung weist seit 1906 folgende verhältnismäßige Zunahmen auf: $8,7,6,4,7,6,7,2$ und 6,6 Prozent.

Die Viehversicherung, deren Änderungen im Bestande hier nur an den Versicherungssummen gemessen werden können, hatte 1906 eine Vermehrung um 4,7 Prozent des vorjährigen Bestandes zu verzeichnen; 1907 brachte eine Abnahme um 1,2 Prozent, 1908 eine Abnahme um 0,92 Prozent, 1909 wieder eine Zunahme um 7,6 Prozent und das Berichtsjahr eine solche um 10,2 Prozent. In der Hagelversicherung brachten die Jahre 1906 bis 1908 bei den Policen Vermehrungen um 6,2, 5,9 und 8,9 Prozent und bei den Summen um 4,7, 3,9 und 4,1 Prozent, wogegen 1909 Abnahmen aufwies, und zwar bei den Policen um 8,6 und bei den Summen um 1,9 Prozent. Im Berichtsjahre nahm die Zahl der Policen um 9,6 Prozent ab und die Versicherungssumme um 1,1 Prozent zu.

Auch die Feuerversicherung, die sich früher besonders regelmäßig entwickelte, hat neuerdings etwas mehr geschwankt. Es hat nämlich in den letzten 5 Jahren die Zunahme der Policenzahl 3,9, 3,7, 4,5, 2,7 und 3,0 Prozent und die Zunahme der Versicherungssummen 4,7, 3,2, 5,9, 4,2 und 4,3 Prozent des Bestandes am Jahresanfange betragen.

Von dem gesamten bei den deutschen Lebensversicherungsgesellschaften Ende 1910 versicherten Kapitale, 13 660,33 (12 820,49) Millionen Mark, kommen 12 512,73 (11 811,49) Millionen Mark auf das deutsche und 1 147,60 (1009,00) Millionen Mark auf das ausländische Geschäft; dagegen hatten die ausländischen Lebensversicherungsgesellschaften zu derselben Zeit im Deutschen Reiche 1 032,76 (1 007,16) Millionen Mark versichert; ihr Anteil an dem gesamten deutschen Kapitalversicherungsgeschäfte, $13545,49(12818,65)$ Millionen Mark, stellt sich danach auf $7,6(7,9)$ Prozent. 
b) Schadenversicherung in den auch direkt betriebenen Zweigen

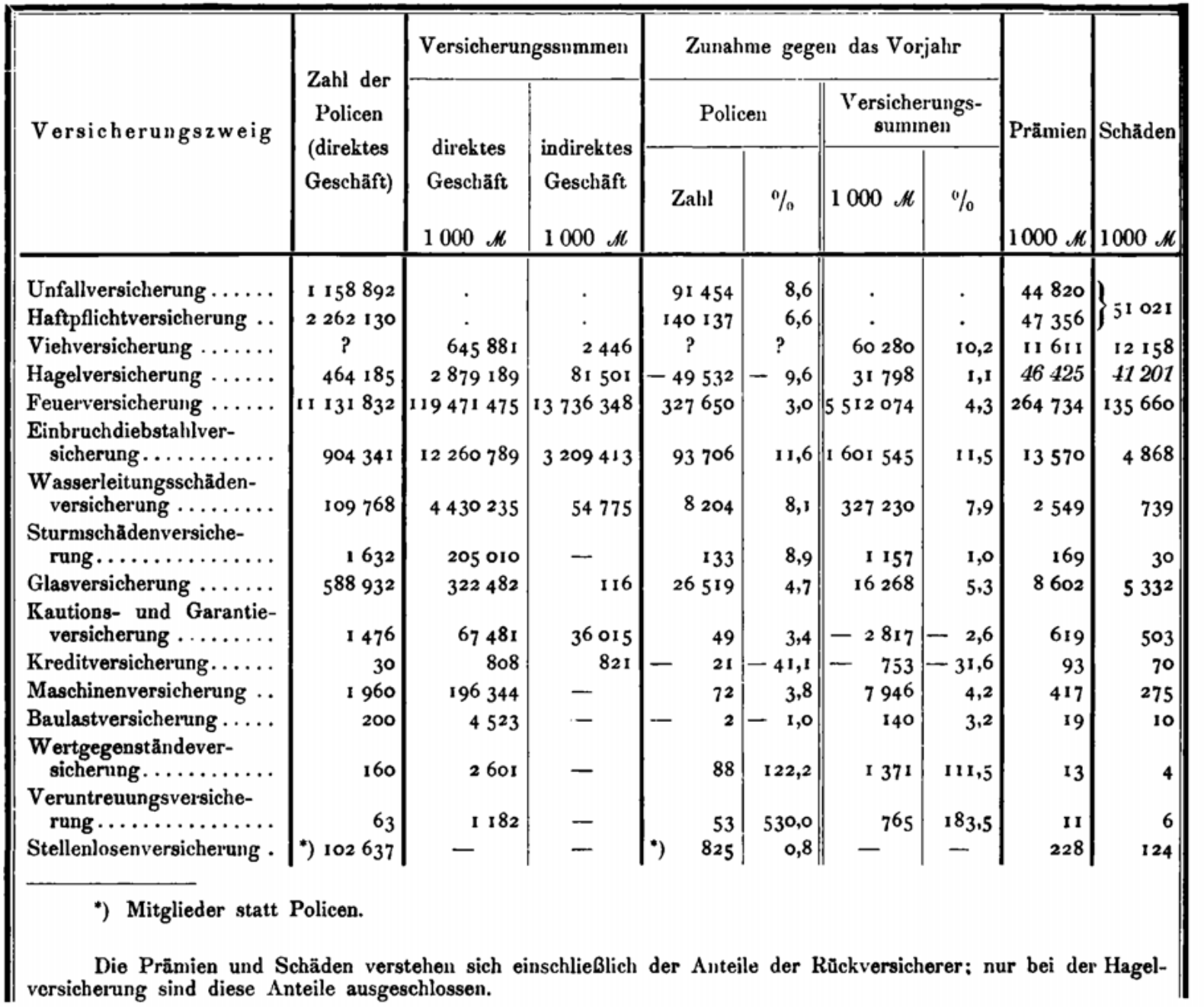

c) Versicherung in ausschließlich indirekt betriebenen Zweigen

\begin{tabular}{|c|c|c|}
\hline Versicherungszweig & $\begin{array}{l}\text { Prämien } \\
\text { für eigene } \\
\text { Rechnung } \\
1000 \mathscr{M}\end{array}$ & $\begin{array}{l}\text { Schäden } \\
\text { für eigene } \\
\text { Rechnung } \\
1000 \mathscr{M}\end{array}$ \\
\hline $\begin{array}{l}\text { Lebensversicherung } \ldots \ldots \ldots \ldots \ldots \\
\text { Unfall- und Haftpflichtversicherung } \\
\text { Hagelversicherung } \ldots \ldots \ldots \ldots \ldots \ldots \\
\text { Feuerversicherung } \ldots \ldots \ldots \ldots \ldots \ldots \\
\text { Sonstige aufsichtspflichtige Zweige. }\end{array}$ & $\begin{array}{r}41231 \\
24503 \\
6135 \\
131319 \\
9163 \\
\end{array}$ & $\begin{array}{r}12385 \\
10901 \\
5740 \\
79752 \\
3266 \\
\end{array}$ \\
\hline Zusammen .... & 212351 & 112044 \\
\hline
\end{tabular}

Beurteilt man in der Unfall- und Haftpflichtversicherung den Umfang des Geschäfts nach der Jahresprämieneinnahme, so kommen von dem mit 92,18 (85,51) Millionen Mark zu bewertenden gesamten Geschäfte der deutschen Gesellschaften $82,68(77,83)$ 
Millionen Mark auf das deutsche und 9,50 (7,68) Millionen Mark auf das ausländische Geschäft; die ausländischen Gesellschaften haben aus ihrem deutschen Geschäfte 16,84 $(16,02)$ Millionen Mark eingenommen; sie sind also an dem gesamten deutschen Geschäfte, 99,52 (93,85) Millionen Mark, mit 16,9 (17,1) Prozent beteiligt gewesen.

In dem direkten Feuerversicherungsgeschäfte hatten die großen deutschen Versicherungsgesellschaften insgesamt 119471 (114586) Millionen Mark versichert; davon kamen 112216 (107 708) Millionen auf das deutsche und 7255 (6 878) Millionen Mark auf das ausländische Geschäft. Die ausländischen Unternehmungen hatten im unmittelbaren deutschen Geschäft um dieselbe Zeit 13018 (12 762) Millionen Mark übernommen. Rechnet man zu dem deutschen Geschäfte noch die Summen, welche nach den "Mitteilungen für die öffentlichen Feuerversicherungs-Anstalten " die im Deutschen Reiche bestehenden 51 öffentlichen Anstalten versichert hatten, nämlich 75627 (72377) Millionen Mark, so hat man in 200861 (192 846) Millionen Mark sehr nahe den ganzen Wert der gegen Feuersgefahr versicherten deutschen Objekte; es fehlen nur die bei den kleinen Vereinen und den unter Landesaufsicht stehenden privaten Gegenseitigkeitsvereinen versicherten Werte, die insgesamt jedoch nur wenige Pro\%ente der soeben angegebenen Summe ausmachen werden. - Von obiger Summe kamen auf die deutschen privaten Unternehmungen unter Reichsaufsicht 55,9 (55,9) Prozent, auf die ausländischen Gesellschaften 6,4 (6,6) Prozent und auf die deutschen öffentlichen Anstalten 37,7 $(37,5)$ Prozent; die Verteilung ist also in beiden Jahren fast genau dieselbe gewesen; sie war auch in den früheren Jahren nur wenig verschieden, vgl. "Entwickelung a S. $\left.2 \mathrm{I}^{*}\right)$.

Aus dem direkten deutschen Geschäfte sind von den sämtlichen in dieser Statistik berücksichtigten privaten Unternehmungen und von den öffentlichen Feuerversicherungsanstalten im Jahre 19 IO für Lebensversicherung 611,16 (j72,18), für Unfall- und Haftpflichtversicherung $96,95(91,34)$ und für Feuerversicherung $302,30(289,67)$, insgesamt 1010,41 $(953,19)$ Millionen Mark an Prämien vereinnahmt worden, das sind rund 2,8 (2,6) Millionen Mark auf den Tag. Die Ausgabe des deutschen Volks für Versicherungen bei privaten Unternehmungen unter Reichsaufsicht hat von I 909 auf I 910 zugenommen bei der Lebensversicherung um 6,8 Prozent, bei der Unfall- und Haftpflichtversicherung um 6,1 Prozent, bei der Feuerversicherung um 4,4 Prozent, bei diesen drei Versicherungszweigen zusammen um 6,0 Prozent; das wird man als einen sehr befriedigenden Fortschritt anerkennen, wenn man bedenkt, daß die durchschnittliche Zunahme der deutschen Bevölkerung in den Jahren I 905 bis I 9 Io 1,4 Prozent im Jahr betragen hat.

Die folgenden Ubersichten sollen einen Einblick in die Betriebsergebnisse des Berichtsjahrs und in den Vermögensstand am Schlusse des Geschäftsjahrs für die Gesamtheit der deutschen Unternehmungen mit Ausnahme der Kaskoversicherungsvereine gewähren.

Hierzu ist folgendes zu bemerken: Es ist schon erwähnt, daß die meisten Aktiengesellschaften mehrere Versicherungszweige betreiben. Die Gesellschaften dieser Art

*) Die Entwickelung des privaten Versicherungsivesens unter Reichsaufsicht in dem Jahrfünft 1902 bis 1906. Herausgegeben vonı Kaiserlichen Aufsichtsamte für Privatversicherung, Berlin 1909, J. Guttentag, Verlagsbuchhandlung, G. m. b. H. - Diese Schrift ist in dem vorliegenden Buche kurz als -Entwickelung“ angeführt. 
geben dann hinsichtlich ihres Lebensversicherungsgeschäfts für die Volksversicherung einerseits und für die gewöhnliche Versicherung einschließlich der "sonstigen kleinen Versicherung " anderseits besondere Gewinn- und Verlustrechnungen, aus denen die folgenden Zahlen abgeleitet worden sind.

\section{Hauptsächlichste Einnahme- und Ausgabeposten der Lebensversicherung}

$1000 \varkappa$

\begin{tabular}{|c|c|c|c|c|c|c|c|c|}
\hline Betriebsart & \begin{tabular}{|} 
Prämien \\
und \\
Police- \\
gebühren
\end{tabular} & $\begin{array}{c}\text { Ver- } \\
\text { gütungen } \\
\text { der } \\
\text { Rückver- } \\
\text { sicherer }\end{array}$ & $\begin{array}{c}\text { Kapital- } \\
\text { erträge } \\
\text { (netto) }\end{array}$ & $\begin{array}{c}\text { Zahlungen } \\
\text { für } \\
\text { Versiche- } \\
\text { rungsfälle }\end{array}$ & $\begin{array}{l}\text { Rück- } \\
\text { versiche- } \\
\text { rungs- } \\
\text { prämien }\end{array}$ & $\begin{array}{c}\text { Zu- } \\
\text { führungen } \\
\text { zu den } \\
\text { Reserven } \\
\text { (netto) }\end{array}$ & \begin{tabular}{|c|} 
Ver- \\
waltungs- \\
kosten und \\
Steuern
\end{tabular} & $\begin{array}{c}\text { Jahres- } \\
\text { über- } \\
\text { schuß }\end{array}$ \\
\hline Volksversicherung*) . . . . . & 107529 & I 834 & 19692 & 39121 & I 826 & $3^{8} 3^{84}$ & 28494 & 20945 \\
\hline $\begin{array}{l}\text { Große Versicherung und - son- } \\
\text { stige kleine Versicherung* }\end{array}$ & 506737 & $2045^{\circ}$ & 172703 & 277408 & 20454 & 213091 & 65825 & 119400 \\
\hline Berufsvereinigungen $\ldots \ldots$ & 11651 & 197 & $335^{\circ}$ & 3263 & 193 & $85 \mathrm{II}$ & 990 & 2438 \\
\hline Zusammen .... & 625917 & 22481 & 195745 & 319792 & 22473 & 259986 & 95309 & 142783 \\
\hline
\end{tabular}

Hinsichtlich der übrigen Versicherungszweige verfahren die Gesellschaften in ihren Rechnungsabschlüssen verschieden; sie trennen entweder alle Einnahme- und Ausgabeposten nach Geschäftszweigen oder nur einen Teil dieser Posten, während sie die übrigen, besonders die Kapitalerträge und die allgemeinen Verwaltungskosten, nur in einer Summe angeben. In den für das Aufsichtsamt bestimmten Aufstellungen werden indessen auch die allgemeinen Posten auf die einzelnen Zweige verteilt; daher kann in dieser Statistik für jeden Zweig der Gewinn aus dem eigentlichen Versicherungsbetrieb angegeben werden. Nur die Unfall- und Haftpflichtversicherung macht eine Ausnahme, weil hier von den Kapitalerträgen ein Teil dem Versicherungsbetriebe zugerechnet werden muß und dieser Teil nicht ausgeschieden werden kann. Aus den bezüglichen Tabellen ist die folgende Ubersicht zusammengestellt worden. Darin sind als Sonstige Betriebs-Einnahmen die Ersparnisse aus der Schadenreserve sowie die Abnahme der Prämienreserven und Uberträge und bei der Viehversicherung der Erlös aus verwertetem Vieh, als Sonstige Betriebs-Ausgaben etwaige Zuschüsse zur Schadenreserve und die Zunahme der Prämienreserven und Überträge, bei der Feuerversicherung auch die Leistungen zu gemeinnützigen Zwecken aufgeführt. Die Anteile der Rückversicherer sind ausgeschlossen. Die Schadenzahlungen enthalten auch die Schadenermittelungskosten.

Die Rückversicherungsgesellschaften haben dem Aufsichtsamt über ihre Geschäftsführung in einer Form zu berichten, die eine Ermittelung des Gewinns aus dem reinen Versicherungsbetriebe weder für einzelne Zweige noch für das gesamte Geschäft gestattet; in die nachstehende Tabelle sind daher als Sonstige Einnahmen und Ausgaben 
die Summen der Jahreseinnahmen (ohne Gewinnvortrag) und Jahresausgaben nach Abzug der Prämien einerseits und der Schäden nebst Verwaltungskosten und Steuern anderseits aus der Gewinn- und Verlustrechnung eingestellt.

\begin{tabular}{|c|c|c|c|c|c|c|}
\hline \multirow[b]{2}{*}{ Versicherungszeig } & \multicolumn{2}{|c|}{ Betriebs-Einnahmen } & \multicolumn{2}{|c|}{ Betriebs-Ausgaben } & \multirow{2}{*}{$\begin{array}{c}\text { Ver- } \\
\text { waltungs- } \\
\text { kosten } \\
\text { und } \\
\text { Steuern }\end{array}$} & \multirow{2}{*}{$\begin{array}{c}\text { Uberschuß } \\
\text { (Verlust -) } \\
\text { des Ver- } \\
\text { sicherungs- } \\
\text { betriebs }\end{array}$} \\
\hline & $\begin{array}{c}\text { Prämien } \\
\text { und Neben- } \\
\text { leistungen } \\
\text { der Ver- } \\
\text { sicherten }\end{array}$ & Sonstige & $\begin{array}{l}\text { Schaden- } \\
\text { zahlungen }\end{array}$ & Sonstige & & \\
\hline Unfall- und Haftpflichtversicherung ... & 91076 & & 45555 & & $33 \mathrm{I} 5 \mathrm{I}$ & \\
\hline Viehversicherung $\ldots \ldots \ldots \ldots \ldots \ldots$ & II 704 & 3397 & I 2255 & - & 2683 & 163 \\
\hline Hagelversicherung $\ldots \ldots \ldots \ldots \ldots \ldots$ & 48425 & - & 42972 & - & 5465 & 12 \\
\hline Feuerversicherung $\ldots \ldots \ldots \ldots \ldots \ldots$ & 161505 & 5359 & 75460 & 9319 & 48014 & 34071 \\
\hline Finbruchdiebstahlversicherung . . ....... & $953^{8}$ & 1410 & $395^{8}$ & 1022 & 3755 & 2213 \\
\hline Wasserleitungsschädenversicherung.... & 2247 & 121 & 706 & 126 & $95 \mathrm{I}$ & 585 \\
\hline Sturnschädenversicherung $\ldots \ldots \ldots \ldots$ & 36 & 1 & 9 & 3 & 12 & 13 \\
\hline Glasversicherung $\ldots \ldots \ldots \ldots \ldots \ldots$ & $865 \mathrm{I}$ & 253 & 5186 & 472 & $273^{6}$ & 510 \\
\hline Kautions- und Garantieversicherung... & 326 & 185 & 363 & 65 & 68 & 15 \\
\hline Kreditversicherung...$\ldots \ldots \ldots \ldots \ldots$ & 47 & 4 & 36 & 2 & 24 & - \\
\hline Maschinenversicherung $\ldots \ldots \ldots \ldots \ldots$ & 115 & 6 & 64 & 2 & 34 & 21 \\
\hline Baulastversicherung $\ldots \ldots \ldots \ldots \ldots$ & 19 & - & II & I & I] & 6 \\
\hline Wertgegenständeversicherung .. & 9 & - & 2 & 3 & 5 & - \\
\hline Veruntreuungsversicherung. . & I & 3 & 2 & - & 5 & - \\
\hline Stellenlosenversicherung....... & 228 & - & 114 & - & 32 & 82 \\
\hline Rückversicherung .......... & 242295 & 22875 & $13+508$ & 48643 & 67732 & 14287 \\
\hline
\end{tabular}

Eine Bilanz wird immer nur für das Gesamtgeschäft aufgestellt und bei derjenigen Gruppe nachgewiesen, zu der die Gesellschaft ihrem aufsichtspflichtigen Hauptbetriebe nach zu rechnen ist. Die Bilanz umfaßt also gegebenenfalls auch das Transportversicherungsgeschäft, über das die Statistik im übrigen nur bei den Rückversicherungsgesellschaften einige Angaben bringt, weil die Transportversicherung der Beaufsichtigung nicht unterliegt. In den Gewinn der Bilanz ist auch der Gewinnvortrag aus dem Vorjahr eingeschlossen.

In der folgenden Tabelle sind berücksichtigt 107 Aktiengesellschaften mit 579,57 Millionen Mark Aktienkapital, wovon 150,07 Millionen Mark eingezahlt sind, und 93 Gegenseitigkeitsvereine mit einem Garantiekapitale von 2,57 Millionen mit 0,96 Millionen Einzahlung. Die für die Aktiengesellschaften in $§ 262$ des Handelsgesetzbuchs und für die Gegenseitigkeitsvereine in $\$ 37$ des Versicherungsaufsichtsgesetzes vorgeschriebenen Kapitalreservefonds beliefen sich Ende I9 IO für alle Unternehmungen zusammen auf 147,0s Millionen; dazu kamen 240,24 Millionen Mark Spezialreserven, die freilich zum Teil nicht als freie Reserven betrachtet werden können. Die Prämienreserven und die Prämienüberträge, 4475,61 Millionen, meist auch die Gewinnreserven, 411,99 Millionen, sowie die Schadenreserven, 152,35 Millionen, sind als gebunden anzusehen, da sie 
Aus der Bilanz der deutschen Unternehmungen für Ende 1910. - Gesamtgeschäft $1000 \mathscr{A}$

\begin{tabular}{|c|c|c|c|c|c|c|c|}
\hline \multirow[b]{2}{*}{ Aktiv- und Passivposten } & \multicolumn{7}{|c|}{ Hauptzweig } \\
\hline & $\begin{array}{l}\text { Lebens- } \\
\text { versiche- } \\
\text { rung }\end{array}$ & $\begin{array}{c}\text { Unfall- } \\
\text { und Haft- } \\
\text { pflicht- } \\
\text { versiche- } \\
\text { rung }\end{array}$ & $\begin{array}{l}\text { Vieh- } \\
\text { versiche- } \\
\text { rung }\end{array}$ & $\begin{array}{c}\text { Hagel- } \\
\text { versiche- } \\
\text { rung }\end{array}$ & $\begin{array}{c}\text { Feuer- } \\
\text { versiche- } \\
\text { rung }\end{array}$ & $\begin{array}{l}\text { Glas- } \\
\text { versiche- } \\
\text { rung }\end{array}$ & $\begin{array}{c}\text { Rück- } \\
\text { versiche- } \\
\text { rung }\end{array}$ \\
\hline Aktiva insgesamt $\ldots \ldots \ldots \ldots \ldots \ldots$ & 5081882 & 248148 & 6379 & 41142 & 625816 & 16886 & 374817 \\
\hline $\begin{array}{c}\text { Verpflichtungen der Aktionäre oder } \\
\text { Garanten } \ldots \ldots \ldots \ldots \ldots \ldots \ldots \ldots\end{array}$ & 121985 & 36479 & - & 18023 & I62 593 & 4086 & 87945 \\
\hline Kapitalanlagen............. & 4718243 & 177,87 & 3769 & 15463 & 378254 & 10400 & 177297 \\
\hline Forderungen $\ldots \ldots \ldots \ldots \ldots \ldots$ & 221837 & 33200 & 2355 & 5250 & 80047 & 2135 & 109048 \\
\hline 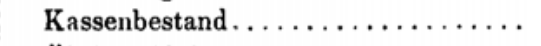 & 6331 & 384 & 149 & 197 & I 549 & 209 & 423 \\
\hline Ubrige Aktiva .............. & 13486 & 898 & 106 & 12 & 3373 & 56 & 104 \\
\hline Verlust $\ldots \ldots \ldots \ldots \ldots \ldots \ldots \ldots$ & - & - & - & 2197 & - & - & - \\
\hline Passiva insgesamt $\ldots \ldots \ldots \ldots \ldots \ldots$ & 5081882 & 248148 & 6379 & 41142 & 625816 & 16886 & 374817 \\
\hline Aktien- oder Garantiekapital ....... & 163577 & 48750 & - & 28529 & 219736 & 5362 & 116186 \\
\hline Kapitalreservefonds ............. & 29701 & 19040 & 2949 & 4657 & 59273 & 983 & 30474 \\
\hline Spezialreserven $\ldots \ldots \ldots \ldots \ldots \ldots$ & 126947 & 24731 & I 35 & 4831 & $5^{8} 488$ & 1364 & 23746 \\
\hline Gewinnreserven.$\ldots \ldots \ldots \ldots \ldots$ & 406203 & 5791 & - & - & - & - & - \\
\hline $\begin{array}{c}\text { Guthaben von Versicherungsunter- } \\
\text { nehmungen...................... }\end{array}$ & 5890 & 13248 & 90 & 494 & 20105 & 64 & 17219 \\
\hline Prämienreserven u. Prämienüberträge & 4107408 & 81706 & I 524 & I & $170 \quad 145$ & 5785 & IO9 045 \\
\hline Schadenreserven ............. & 26662 & $3673^{8}$ & 484 & 420 & 28723 & 1623 & 57697 \\
\hline Ubrige Passiva ........... & $6944 \mathrm{I}$ & 3886 & I 108 & 2210 & 19700 & 923 & $35^{87}$ \\
\hline 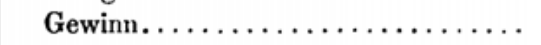 & 146053 & 14258 & 89 & - & 49646 & 782 & 16863 \\
\hline
\end{tabular}

als rechnungsmäßige Deckung für die teils bereits entstandenen, teils voraussichtlich in Zukunft noch entstehenden Ansprüche der Versicherten aus geleisteten Prämienzahlungen dienen.

Die Kapitalanlagen aller dieser 200 Unternehmungen haben Ende 1910 insgesamt 5 480,61 Millionen Mark betragen; davon entfallen 141,00 Millionen auf Grundbesitz, 4 347,22 Millionen auf Hypotheken und Grundschulden, 194,85 Millionen auf Darlehen an öffentliche Körperschaften, 4,79 Millionen auf Darlehen auf Wertpapiere, 431,23 Millionen auf Wertpapiere, 343,09 Millionen auf Policedarlehen, 14,70 Millionen auf Wechsel und 3,73 Millionen auf sonstige Anlagen.

Die Verteilung auf diese Wertarten ist aber bei den verschiedenen Versicherungszweigen und zum Teil auch bei den beiden Geschäftsformen eine recht verschiedene, auch wird sie durch heimische Übung und Gesetzgebung mannigfach beeinflußt. Darauf ist bereits in den Statistiken für 1903 und 1904 und in der "Entwickelung " S. 45 hingewiesen worden. 
Kapitalanlagen Ende 1910 (Bilanzwert)

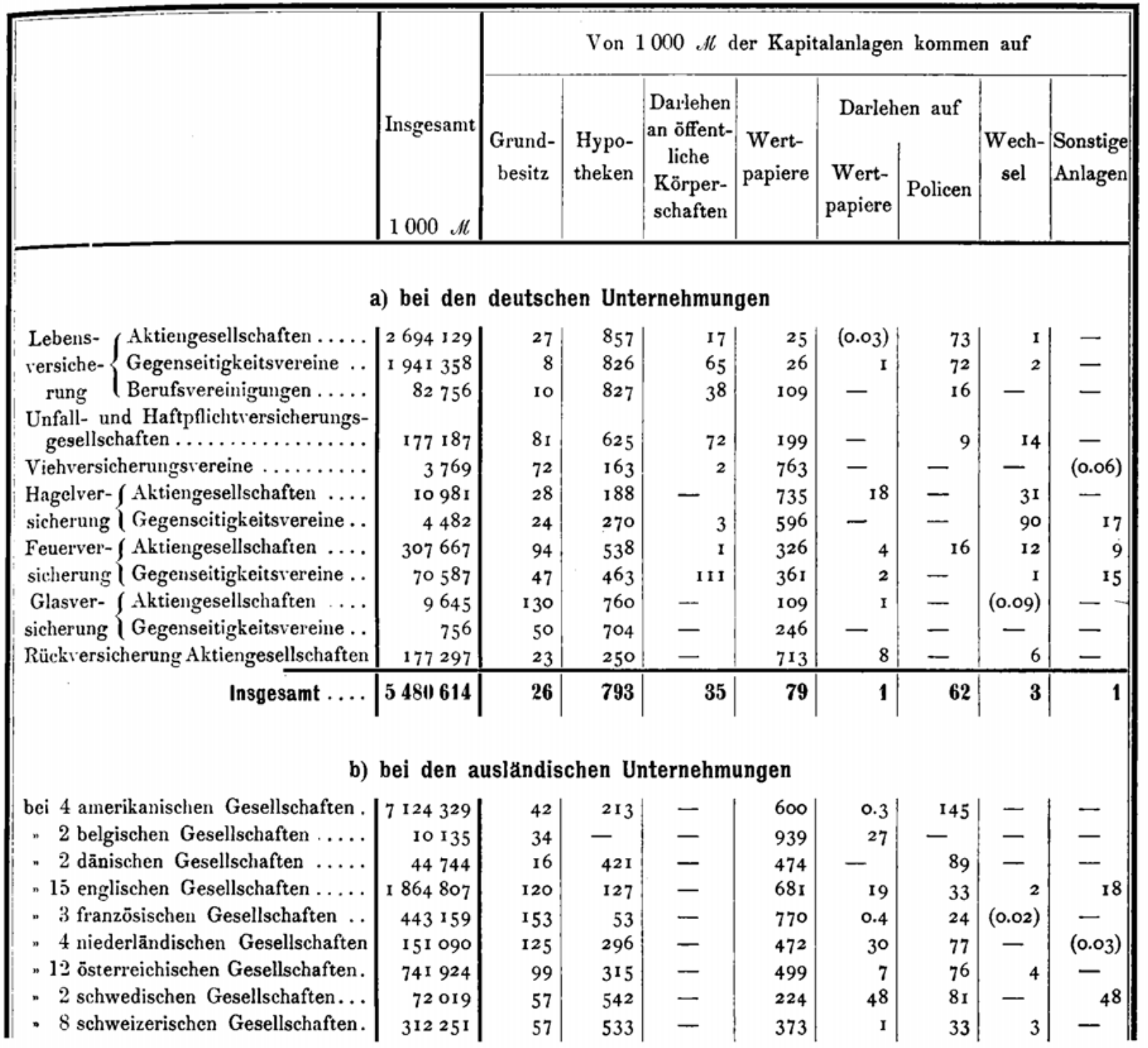

Die Kapitalanlagen der Versicherungsunternehmungen bilden noch immer den Gegenstand öffentlicher Verhandlungen; dabei wird neben dem Verhältnisse des Wertpapierbestandes zu den gesamten Anlagen auch die Art der im Besitze der Unternehmungen befindlichen Papiere in die Erörterung gezogen. Es soll daher auch in diesem Jahre eine Zusammenstellung, die hierüber näheren Aufschluß gibt, mitgeteilt werden. Zu bemerken ist nur, daß der Verteilung der Nennwert zugrunde gelegt worden ist und daß der bei den einzelnen Zweigen eingestellte Nennwert des Gesamtbestandes wegen besonderer Umstände, die aus den Fußnoten zu den betreffenden Tabellen ersichtlich sind und hier nicht angeführt zu werden brauchen, nicht überall genau dem bei den Kapitalanlagen in der Bilanz verrechneten Werte entspricht; die Abweichungen sind übrigens so gering, daß sie hier nicht ins Gewicht fallen. 
Verteilung der Wertpapiere (Nennwert)

\begin{tabular}{|c|c|c|c|c|c|c|c|c|c|}
\hline & \multirow{3}{*}{$\mid \begin{array}{c}\text { Wert- } \\
\text { papiere } \\
\text { ins- } \\
\text { gesamt } \\
1000 \text { }\end{array}$} & \multicolumn{8}{|c|}{ Von $1000 \mathscr{A l}$ der Wertpapiere kommen auf } \\
\hline & & \multicolumn{5}{|c|}{ inländische Wertpapiere } & \multicolumn{3}{|c|}{ ausländische Wertpapiere } \\
\hline & & \begin{tabular}{|} 
Staats- \\
anleihen \\
und \\
staatlich \\
garan- \\
tierte \\
Anleihen
\end{tabular} & $\begin{array}{l}\text { Kom- } \\
\text { munal- } \\
\text { anleihen }\end{array}$ & $\begin{array}{c}\text { Pfand- } \\
\text { briefe } \\
\text { und } \\
\text { Kommu- } \\
\text { nalobli- } \\
\text { gationen }\end{array}$ & \begin{tabular}{|c|} 
sonstige \\
Schuld- \\
ver- \\
schrei- \\
bungen
\end{tabular} & Aktien & $\begin{array}{c}\text { Staat- } \\
\text { liche und } \\
\text { komnu- } \\
\text { nale } \\
\text { Anleihen }\end{array}$ & $\begin{array}{l}\text { sonstige } \\
\text { Schuld- } \\
\text { ver- } \\
\text { schrei- } \\
\text { hungen }\end{array}$ & Aktien \\
\hline I.ebens- (Aktiengesellschaften ..... & $7 \mathrm{I} 065$ & 479 & 46 & r 39 & 4 & - & 323 & 4 & 5 \\
\hline versiche- $\{$ Gegenseitigkeitsvereine $\ldots$ & $5544 \mathrm{I}$ & 529 & 185 & 148 & - & - & 134 & 4 & - \\
\hline rung $\quad$ Berufsvereinigungen ...... & 9412 & 472 & 275 & 242 & 10 & 0,2 & 1 & - & - \\
\hline $\begin{array}{l}\text { Unfall- und Haftpflichtversicherungs- } \\
\text { gesellschaften ..................... }\end{array}$ & $3^{8} 375$ & 530 & 113 & $5^{8}$ & I & 45 & 154 & 94 & 5 \\
\hline Viehversicherungsvereine..$\ldots \ldots \ldots$ & 3027 & 480 & 243 & 272 ! & I & - & 4 & - & - \\
\hline Hagelver- $($ Aktiengesellschaften .... & 7559 & 618 & - & 3 & 8 & 366 & 5 & - & - \\
\hline sicherung Gegenseitigkeitsvereine .. & 2940 & 363 & 89 & 548 & - & - & - & - & - \\
\hline Feuerver- Aktiengesellschaften .... & 108913 & 560 & $5^{8}$ & 103 & 3 & Io & 154 & 108 & 4 \\
\hline sicherung $\{$ Gegenseitigkeitsvereine.. & 28329 & 424 & 301 & 234 & 2 & $0, \mathbf{r}$ & 21 & 18 & - \\
\hline Glasver- ( Altiengesellschaften .... & I 171 & 502 & 28 & 67 & - & 64 & 338 & I & - \\
\hline sicherung Gegenseitigkeitsvereine... & 197 & 442 & 30 & 325 & 195 & - & 8 & - & - \\
\hline Rückversicherung Aktiengesellschaften & $\mathrm{I} 5.309 \mathrm{I}$ & $34 \mathrm{I}$ & 47 & 50 & 7 & $\mathrm{II}_{3}$ & 103 & 202 & $\mathbf{1} 37$ \\
\hline Insgesamt .... & 479520 & 461 & 91 & 106 & 4 & 48 & 146 & 98 & 46 \\
\hline
\end{tabular}

Die Verteilung des Gewinns
In der obigen Bilanz-Tabelle sind bei jedem Versicherungszweige die Verluste, die einige Unternehmungen zu verzeichnen hatten, gegen die Gewinne der anderen Gesellschaften verrechnet. Es ergibt sich so für die Gesamtheit der deutschen Versicherungsunternehmungen ein Netto-Gewinn von 227,691 Millionen Mark. Handelt es sich aber um die Verteilung des Gewinns, so kommt der durch die Bilanz ausgewiesene Gewinn aller derjenigen Gesellschaften in Betracht, die mit finanziell günstigem Erfolge gearbeitet haben, das sind 228,540 Millionen Mark. Wie dieser Betrag verteilt worden ist, zeigt folgende Übersicht:

\begin{tabular}{|c|c|c|c|c|c|c|c|c|}
\hline $\begin{array}{c}\text { Von dem Gewinne sind in } 1000 \mathscr{A} \\
\text { abgeführt worden }\end{array}$ & $\begin{array}{l}\text { an den } \\
\text { Kapital- } \\
\text { reserve- } \\
\text { fonds }\end{array}$ & $\begin{array}{c}\text { an } \\
\text { sonstige } \\
\text { Reserven }\end{array}$ & $\begin{array}{c}\text { an den } \\
\text { Beamten- } \\
\text { fonds }\end{array}$ & $\begin{array}{c}\text { an die } \\
\text { Aktionäre } \\
\text { oder } \\
\text { Garanten }\end{array}$ & $\begin{array}{l}\text { an Tan- } \\
\text { tieme- } \\
\text { be- } \\
\text { rechtigte }\end{array}$ & $\begin{array}{c}\text { an die } \\
\text { Ver- } \\
\text { sicherten }\end{array}$ & $\begin{array}{c}\text { ander- } \\
\text { weit }\end{array}$ & $\begin{array}{l}\text { Vortrag } \\
\text { auf neue } \\
\text { Rechnung }\end{array}$ \\
\hline Lebensversicherung............ & I 339 & $855^{8}$ & I 302 & 7328 & 3329 & $124 \times 82$ & 792 & 573 \\
\hline Unfall- und Haftpflichtversicherung. & 38 & 957 & 299 & 4237 & 1263 & 3231 & $68 \mathrm{I}$ & 2214 \\
\hline Viehversicherung $\ldots \ldots \ldots \ldots \ldots$ & 39 & 16 & - & - & 3 & 30 & I & - \\
\hline Hagelversicherung ............. & 93 & 165 & - & 346 & 36 & - & - & 28 \\
\hline Feuerversicherung usw. .......... & I 930 & 5394 & 952 & 14195 & 2296 & 20468 & I 889 & $255^{2}$ \\
\hline Glasversicherung $\ldots \ldots \ldots \ldots \ldots$ & 59 & 6 & 26 & 354 & I 59 & 28 & 112 & $3^{8}$ \\
\hline Rückversicherung $\ldots \ldots \ldots \ldots \ldots$ & 280 & 4551 & 222 & 7078 & 917 & - & 170 & 3784 \\
\hline Zusammen .... & 3788 & 19647 & 2801 & $3: 538$ & 8003 & 147939 & 3645 & 9189 \\
\hline
\end{tabular}


$\Lambda u ß e r d e m$ sind den Versicherten der Lebensversicherungsabteilungen verschiedener Aktiengesellschaften zusammen noch 3,188 Millionen vor dem Abschluß der Jahresrechnung als Dividende überwiesen worden, die in vorstehender Tabelle nicht berücksichtigt sind.

Schließlich mag hier noch eine Gegenüberstellung der Prämien und der Schäden der direkt arbeitenden Unternehmungen, beide einschließlich der Anteile der Rückversicherer und des indirekten Geschäfts, bei den verschiedenen Versicherungszweigen Plat\% finden, die einerseits das ausländische Geschäft der deutschen Gesellschaften und andererseits das deutsche Geschäft der ausländischen Gesellschaften betreffen. Es sei aber besonders hervorgehoben, daß aus den Endzahlen keineswegs Schlüsse auf die Verhältnisse des Versicherungsgeschäfts überhaupt gezogen werden dürfen; dazu fehlen die Zahlen für die ganz vornehmlich internationalen Betriebe der Transportversicherung und der Rückversicherung.

\begin{tabular}{|c|c|c|c|c|}
\hline \multirow{2}{*}{ Versicherungszweig } & \multicolumn{2}{|c|}{$\begin{array}{l}\text { Ausländisches Geschäft der } \\
\text { deutschen Gesellschaften }\end{array}$} & \multicolumn{2}{|c|}{$\begin{array}{l}\text { Deutsches Geschäft der aus- } \\
\text { ländischen Gesellschaften }\end{array}$} \\
\hline & $\begin{array}{l}\text { Prämien } \\
1000 \mathscr{A t}\end{array}$ & $\begin{array}{l}\text { Schäden } \\
1000 \text { ॥t }\end{array}$ & $\begin{array}{l}\text { Prämịen } \\
1000 \varkappa\end{array}$ & $\begin{array}{l}\text { Schäden } \\
1000 \mathscr{A}\end{array}$ \\
\hline 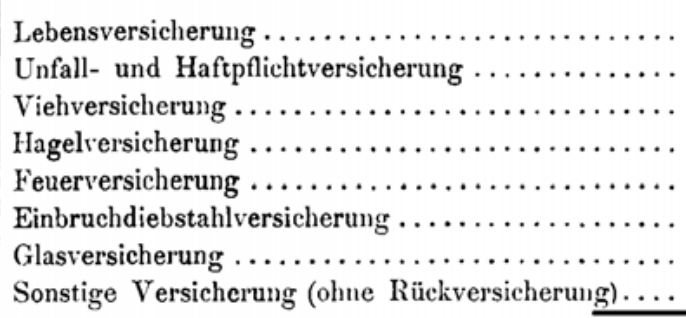 & $\begin{array}{r}51 \\
9502 \\
176 \\
379 \\
70671 \\
1921 \\
672 \\
147 \\
\end{array}$ & $\begin{array}{r}21182 \\
5634 \\
130 \\
301 \\
41523 \\
1722 \\
401 \\
266 \\
\end{array}$ & $\begin{array}{r}43987 \\
16836 \\
-\quad \\
-\quad \\
28054 \\
886 \\
172 \\
200 \\
\end{array}$ & $\begin{array}{r}36949 \\
7704 \\
-\quad \\
-\quad \\
13824 \\
357 \\
121 \\
148 \\
\end{array}$ \\
\hline $\begin{array}{r}\text { Zusammen } \ldots . \\
\\
\begin{array}{r} \\
\text { Im Jahre } 1909 \ldots \\
1908 \ldots \\
1907 \ldots \\
1906 \ldots \\
1905 \ldots \\
1904 \ldots \\
1903 \ldots \\
1902 \ldots\end{array}\end{array}$ & $\begin{array}{r}134647 \\
121230 \\
112975 \\
109753 \\
108184 \\
99917 \\
97037 \\
94726 \\
90930\end{array}$ & $\begin{array}{r}71159 \\
68710 \\
65840 \\
60002 \\
125814 \\
54731 \\
55273 \\
51971 \\
54163\end{array}$ & $\begin{array}{ll}90 & 135 \\
& \\
87 & 457 \\
84 & 790 \\
81 & 942 \\
78 & 274 \\
75 & 837 \\
72 & 879 \\
68 & 393 \\
6 & 1383\end{array}$ & $\begin{array}{l}59103 \\
\\
58772 \\
55180 \\
55344 \\
48463 \\
45586 \\
44555 \\
37103 \\
33219\end{array}$ \\
\hline
\end{tabular}

\section{Gruppe I, Lebens- und Krankenversicherung}

Im Jahre 910 standen auf dem Gebiete der Lebensversicherung und der Kranken- Die Lnterversicherung 28 deutsche Aktiengesellschaften und eine große Zahl deutscher Gegenseitigkeitsvereine unter Reichsaufsicht.

Während 28 Aktiengesellschaften und 15 große Gegenseitigkeitsvereine ihren Geschäftsbetrieb für alle Kreise der Bevölkerung offen halten, versichert die Mehrzahl 
der Gegenseitigkeitsvereine nur die Angehörigen mehr oder weniger eng begrenzter Kreise. Von diesen Vereinen hat nur einer (der Preußische Beamtenverein in Hannover) einen so großen Versicherungsbestand gewonnen, daß man ihn den anderen Gegenseitigkeitsvereinen, die eine Beschränkung auf bestimmte Kreise nicht kennen, an die Seite stellen kann; deshalb ist er auch hier jenen allgemeinen Vereinen zugesellt worden. Von den anderen Gegenseitigkeitsvereinen für beschränkte Personengruppen sind in die Tabellen dieser Statistik nur einige der größeren Versicherungseinrichtungen von Berufsvereinigungen aufgenommen. Die große Mehrzahl von Pensions- und Sterbekassen, die vielfach nur die Versicherung der Angestellten bestimmter gewerblicher Firmen bezwecken, sind wie in den früheren Jahren aus den Tabellen ganz fortgelassen; sie werden weiter unten nur im Texte ganz zusammenfassend behandelt.

Auch die größeren Pensions- und Sterbekassen gehören ihrem ganzen Betriebe nach eher zu der kleinen als zu der großen Versicherung; sie sind hier aber gesondert behandelt und als Versicherungseinrichtungen von Berufsvereinigungen zusammengefaßt. Die Bezeichnung "kleine Versicherung “ ist im folgenden lediglich für die kleine Versicherung der im allgemeinen allen Bevölkerungsklassen zugänglichen großen Unternehmungen verwendet.

Von allen dem Aufsichtsamt unterstehenden deutschen Lebensversicherungsvereinen auf Gegenseitigkeit sind in den Tabellen dieser Statistik 16 größere Gegenseitigkeitsvereine allgemeinen Charakters und 18 Versicherungseinrichtungen von Berufsvereinigungen behandelt.

Eine Aktiengesellschaft - Nordstern, Unfall- und Haftpflicht-Versicherungs-AktienGesellschaft in Berlin - hat sich, soweit Versicherungen der Gruppe I in Frage kommen, nur an der kleinen Versicherung beteiligt.

Die Bremer Lebensversicherungs-Bank, Aktiengesellschaft und die Hannoversche Lebensversicherungs-Anstalt auf Gegenseitigkeit haben sich unter der Firma BremenHannoversche Lebensversicherungs-Bank, Aktiengesellschaft, mit dem Sitze in Hannover vereinigt. Die übrigen Änderungen gegenüber dem Vorjahre betreffen lediglich die Firmenbezeichnung: Nordstern, Unfall- und Alters-Versicherungs-Aktien-Gesellschaft zu Berlin zeichnet jetzt Nordstern, Unfall- und Haftpflicht-Versicherungs-Aktien-Gesellschaft zu Berlin und die Hinterbliebenen- und Pensions-Versicherungsanstalt des Verbandes Deutscher Beamtenvereine auf Gegenseitigkeit hat den Namen Deutsche BeamtenLebensversicherung a. G., Anstalt des Verbandes Deutscher Beamtenvereine in Berlin angenommen.

Von ausländischen Lebensversicherungsgesellschaften haben im Jahre I910 wie im vorigen Jahre 21 der Reichsaufsicht unterstanden.

Von den 28 deutschen Aktiengesellschaften betrieben im Jahre 1910 neben der Lebensversicherung 14 auch die Unfallversicherung, 13 die Haftpflichtversicherung, 3 die Feuerversicherung, 3 die Kautionsversicherung (in ganz bescheidenem Umfang), 2 die Transportversicherung und 3 die Versicherung gegen Einbruchdiebstahl. Von den Gegenseitigkeitsvereinen hat nur einer (der Allgemeine Deutsche Versicherungs-Verein in Stuttgart) außer in der Lebensversicherung noch in der Unfall- und Haftpflichtversicherung und in einigen kleinen Nebenzweigen Gesehäfte gemacht. 
Von den allen Kreisen der Bevölkerung zugänglichen deutschen Unternehmungen unter Reichsaufsicht sind im Jahre 1910 tätig gewesen

in der großen Versicherung

in der Versicherung auf den Todesfall 27 Aktiengesellschaften und 14 Gegenseitigkeitsvereine, zus. 4I Unternehmungen

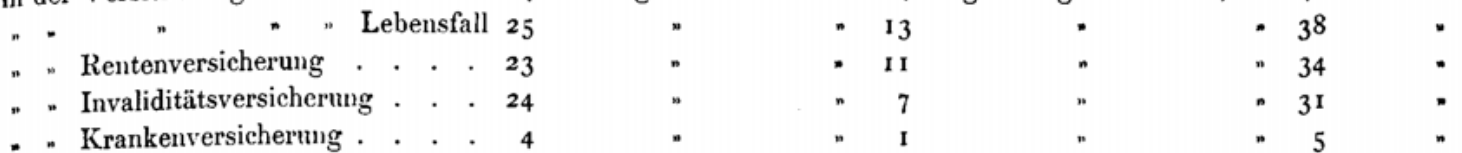

in der kleinen Versicherung

in der Volksversicherung . . . I I Aktiengesellschaften und 2 Gegenseitigkeitsvereine, zusammen 13 Unternehmungen in der sonstigen kleinen Vers. . I5

Von den 21 ausländischen Gesellschaften, über welche diese Statistik berichtet, haben im Deutschen Reiche gearbeitet sämtliche in der großen Versicherung auf den Todesfall und in der Versicherung auf den Lebensfall, 18 in der Rentenversicherung, 3 in der Invaliditätsversicherung und 4 in der Volksversicherung.

Vom Standpunkte der Statistik ist eine möglichst vollständige Trennung des großen Geschäfts von dem kleinen Geschäfte wünschenswert; denn der Betrieb ist verschieden, und die Versicherungen dienen zum 'Teil verschiedenen Zwecken.

Nun haben nach den Rechnungsvorschriften diejenigen Versicherungsunternehmungen, deren Geschäftsbetrieb die sogenannte kleine Lebensversicherung (Volksversicherung, Arbeiter-, Sterbekassen- usw. Versicherung) ohne ärztliche Untersuchung oder mit unvollständiger ärztlicher Untersuchung in der Art umfaßt, daß für diesen Versicherungsbetrieb besondere geschäftliche Einrichtungen und besondere, von den sonstigen Geschäftsgrundsätzen der Unternehmung wesentlich abweichende allgemeine Versicherungsbedingungen, Tarife und Provisionssätze eingeführt sind, für diesen Versicherungsbetrieb besondere Nachweisungen, namentlich auch über den Versicherungsbestand und seine Bewegung sowie linsichtlich der Gewinn- und Verlustrechnung, aufzustellen. Sofern aber die Unternehmungen für diesen Versicherungsbetrieb keine besonderen Einrichtungen haben, sind sie berechtigt, die kleine Versicherung mit der großen zusammen nachzuweisen.

Unter diesen Umständen ist es nicht möglich gewesen, die kleine Versicherung vollständig aus dem gesamten Geschäfte herauszuschälen. Bei einigen Unternehmungen konnte die Trennung nur bei dem Versicherungsbestande, nicht aber auch bei der Bewegung des Bestandes vollständig durchgeführt werden. Wo die Scheidung mit Hilfe der dem Aufsichtsamt eingereichten Ubersichten oder der gedruckten Geschäftsberichte möglich war, ist sie vorgenommen worden, obwohl dadurch eine gewisse Ungleichmäßigkeit zwischen den einzelnen Abschnitten dieser Statistik entstehen mußte; es erschien besser, diese Ungleichmäßigkeit in den Kauf zu nehmen, als auf eine strenge Scheidung der beiden Betriebsarten vollständig zu verzichten. Da, wo die Unternelımungen für die kleine Versicherung besondere geschäftliche Einrichtungen obengenannter Art geschaffen haben und wo mithin das kleine Geschäft von dem großen vollständig getrennt gehalten werden kann, wird in dieser Statistik die kleine Versicherung als "Volksversicherung ", wo das nicht der Fall ist, als "sonstige kleine Versicherung " bezeichnet. 
Die Rothenburger Versicherungs-Anstalt hat bisher nur kleine Versicherungen abgeschlossen, doch kann das Geschäft nach seiner ganzen Natur nicht wohl zur Volksversicherung gerechnet werden; es sind daher der Versicherungsbestand und seine Bewegung bei der "sonstigen kleinen Versicherung " und die Betriebsergebnisse bei der großen Versicherung nachgewiesen.

Die Vereins-Versicherungs-Bank in Düsseldorf hat für das Berichtsjahr nur den Versicherungsbestand, aber nicht auch die Betriebsrechnung getrennt für große und kleine Versicherungen aufgestellt. Da sie das Hauptgewicht auf die kleine Versicherung legt, ist ihre Betriebsrechnung für das ganze Geschäft hier bei der Volksversicherung nachgewiesen.

Anordnung des Stofres

(Tabellen I 1 bis I 18); es folgt die Bewegung des Versicherungsbestandes im Berichtsjahre (Tabellen I 19 bis I 28), dann die Gewinn- und Verlustrechnung (Tabellen I 29 bis I 37) und endlich die Bilanz für Ende 19 Io (Tabellen I 38 bis I 48).

In den einzelnen Abschnitten werden nacheinander berücksichtigt: die großen deutschen Unternehmungen (Aktiengesellschaften und Gegenseitigkeitsvereine), zuerst mit ihrer großen, dann mit ihrer kleinen Versicherung, danach die Versicherungseinrichtungen einiger größerer deutscher Berufsvereinigungen (Pensionskassen und Sterbekassen) und endlich die ausländischen Unternehmungen. Dabei wird von den deutschen Unternehmungen in den ersten drei Abschnitten immer das Lebensversicherungsgeschäft (inländisches und ausländisches) gesondert betrachtet, während die Bilanz nur für das Gesamtgeschäft (Lebensversicherung nebst allen Nebenzweigen) gegeben wird. Von den ausländischen Unternehmungen wirl der Bestand und die Bewegung nur für das deutsche Geschäft gegeben; dagegen bezieht sich die Betriebsrechnung auf das ganze Lebensversicherungsgeschäft und die Bilanz auf das Gesamtgeschäft.

Hieran schließt sich eine Vergleichung des ausländischen Geschäfts der deutschen Unternehmungen mit dem deutschen Geschäfte der ausländischen Gesellschaften in den wichtigsten Punkten; endlich folgen einige Angaben über die dem Aufsichtsamt unterstehenden kleinen Pensions- und Sterbekassen.

\section{Der Versicherungsbestand am Ende des Jahres 1910}

Deutsche

allgemeine

Anstalten

versicherungsunternehmungen, die dem großen Publikum dienen, geben die Tabellen I 1
bis I 10 ausführlichen Aufschluß; hier sollen nur die hauptsächlichsten, für die Ge-
samtheit der Anstalten geltenden Ergebnisse mitgeteilt werden. 


\begin{tabular}{|c|c|c|c|c|c|c|}
\hline \multirow{2}{*}{ Versicherungsart } & \multicolumn{3}{|c|}{$\begin{array}{l}\text { C'berhaupt übernommene Versicherungen } \\
\text { in } 1000 \mathscr{A}\end{array}$} & \multicolumn{3}{|c|}{$\begin{array}{l}\text { Von } 1000^{\circ} \mathscr{A l} \text { überhaupt über- } \\
\text { nommener Vers. wurden für } \\
\text { eigene Rechnung behalten }\end{array}$} \\
\hline & $\begin{array}{l}\text { Aktien- } \\
\text { gesell- } \\
\text { schaften }\end{array}$ & $\begin{array}{l}\text { Gegen- } \\
\text { seitigkeits- } \\
\text { rereine }\end{array}$ & zusammen & $\begin{array}{l}\text { Aktien- } \\
\text { gesell- } \\
\text { schaften }\end{array}$ & \begin{tabular}{|c|} 
Gegen- \\
seitigkeits- \\
vereine
\end{tabular} & zusammen \\
\hline Grofie Versicherung & & & & & & \\
\hline Versicherung auf den Todesfall....... & $6177 \mathrm{101}$ & 4867818 & 11044919 & 924 & 991 & 954 \\
\hline$" \quad, \quad$ Lebensfall...... & 423332 & 396035 & 819367 & 985 & 1000 & 992 \\
\hline $\begin{array}{l}\text { Rentenversicherung................. } \\
\qquad \text { Kleine Versicherung }\end{array}$ & 20395 & 5707 & 26102 & 947 & 999 & $95^{8}$ \\
\hline Volksversicherung auf den Todesfall ... & 1295654 & 63960 & 1359614 & $\begin{array}{l}981 \\
826\end{array}$ & 1000 & 982 \\
\hline Sonstige kleine Versicherung...$\ldots \ldots$ & 44726 & 127332 & $17205^{8}$ & $\begin{array}{l}020 \\
990\end{array}$ & 1000 & 997 \\
\hline
\end{tabular}

Die Rückversicherung spielt hiernach in der Lebensversicherung im ganzen nur eine bescheidene Rolle, zumal bei den Gegenseitigkeitsvereinen, bei denen sie überhaupt nur in der großen Kapitalversicherung auf den Todesfall in Betracht kommt. Bei der Lebensfall- und der Rentenversicherung sowie bei der kleinen Versicherung tritt sie eigentlich nur da auf, wo Gesellschaften vertragsmäßig verpflichtet sind, von jeder von ihnen abgeschlossenen Versicherung einen bestimmten Prozentsatz in Rückdeckung zu geben (sogenannte Quotenrückversicherung).

Fragt man dann weiter, wie sich der Versicherungsbestand der deutschen Gesellschaften aus selbst abgeschlossenen und aus in Rückdeckung übernommenen Versicherungen zusammensetzt, so ersieht man aus den Tabellen I 3 bis I $\tau$ und I 9 und 10 , daß die Gegenseitigkeitsvereine Ende I9 10 nur vereinzelt einige Versicherungen auf den Todesfall und geringfügige Jahresrenten in Rückdeckung genommen und daß auch die Aktiengesellschaften im großen und ganzen nur wenig mittelbare Geschäfte abgeschlossen haben.

In der großen Todesfallversicherung haben die Unternehmungen noch nicht einmal halb so viel Versicherungen in Rückdeckung übernommen, wie sie in Rückdeckung gegeben haben; bei den anderen großen Versicherungen treten die in Rückdeckung übernommenen Beträge noch mehr zurück.

In der Volksversicherung ist das indirekte Geschäft überhaupt kaum nennenswert.

Besondere Beachtung verdienen die Zahlen, die sich auf dre direkten, selbst abgeschlossenen Versicherungen beziehen, weil darin ein Maßstab für den Versicherungsbedarf der Bevölkerung gefunden werden kann. Wir geben davon sowohl die Zahl der e) das unmittelbare Geschaft Policen als auch die Summen und die durchschnittlich auf eine Police entfallenden Beträge und setzen zum Vergleiche die Zahlen für I905 daneben. 
Gruppe I

Direktes Geschäft der deutschen allgemeinen Unternehmungen

Versicherungssummen in $1000 \mathscr{M}$, Jahresrenten und Durchschnittsbeträge in $\mathscr{N}$

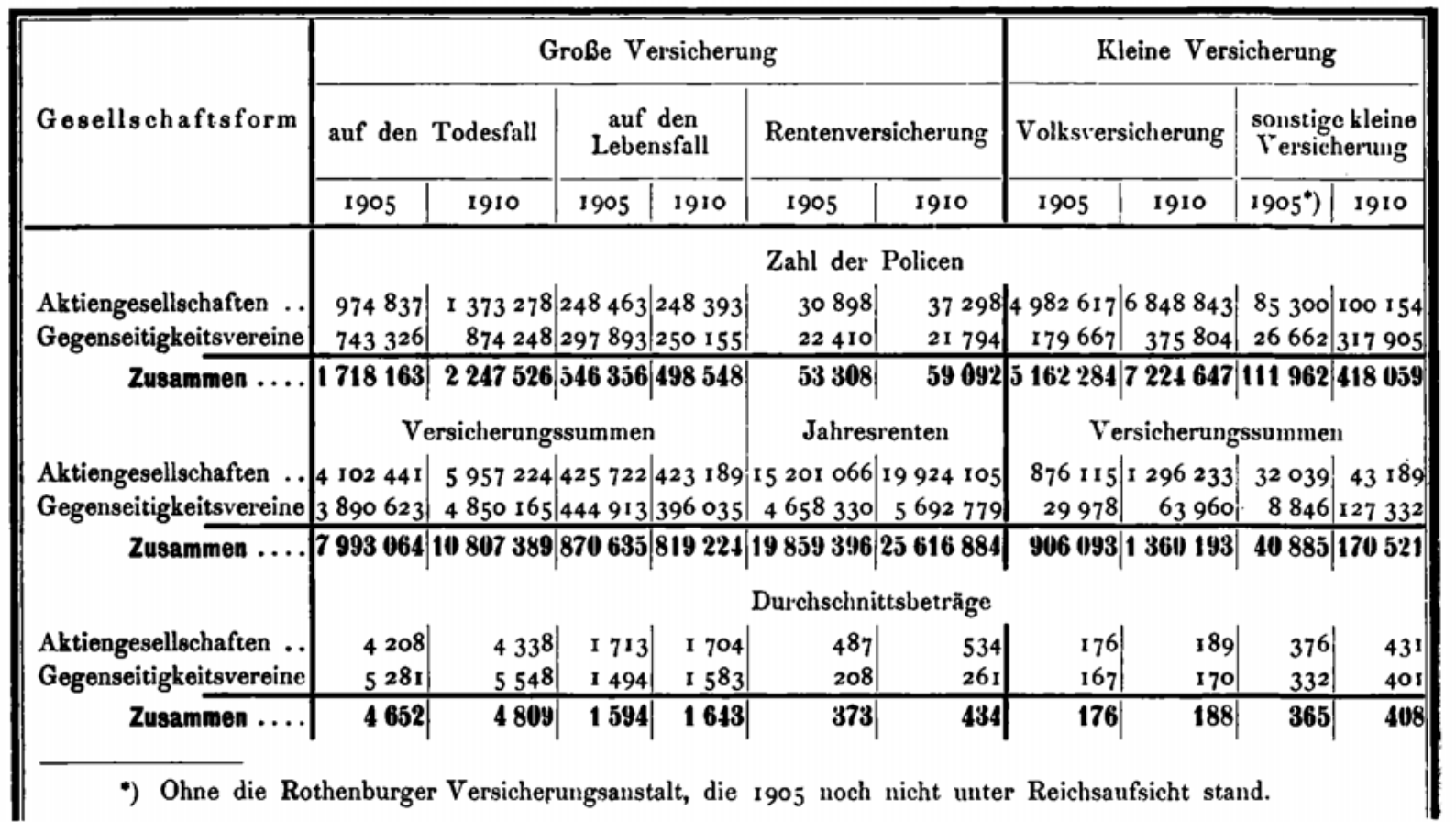

Die durchschnittlich auf eine Police entfallende Versicherungssumme ist in der großen Todesfallversicherung sowohl bei den Aktiengesellschaften wie auch bei den Gegenseitigkeitsvereinen gewachsen; sie ist bei den Gegenseitigkeitsvereinen fortdauernd höher als bei den Aktiengesellschaften. Der auf eine Versicherung auf den Lebensfall kommende durchschnittliche Betrag ist umgekehrt bei den Aktiengesellschaften größer als bei den Gegenseitigkeitsvereinen; während der Durchschnittsbetrag bei den Aktiengesellschaften in den letzten Jahren etwas geschwankt hat, hat er bei den Gegenseitigkeitsvereinen ohne Unterbrechung zugenommen.

d) die Versicherun. gen mit and ohne Gewinnbeteiligung
In den Tabellen I 3 bis 7 und I 9 und 10 ist auch die Gewinnbeteiligung bei dem direkten Geschäfte behandelt. Daß Versicherungen mit Gewinnbeteiligung bei den Aktiengesellschaften verhältnismäßig weniger vorkommen als bei den Gegenseitigkeitsvereinen, liegt in der Natur der Gesellschaften. Genaueres hierüber ergibt für die letzten fünf Jahre die folgende Zusammenstellung.

Diese Zahlen zeigen durchweg einen sehr regelmäßigen Verlauf. In der Lebensfallversicherung und in der Rentenversicherung der Gegenseitigkeitsvereine sind die Versicherungen mit Gewinnbeteiligung während des betrachteten Zeitraums verhältnismäßig zurückgegangen, sowohl was die Zahl der Versicherungen als auch was die Höhe der Versicherungssummen betrifft. Bei den Aktiengesellschaften zeigen sich neuerdings einige Schwankungen. In der großen Versicherung auf den 'Todesfall ist der Anteil der gewinnberechtigten Versicherungen bei den Gegenseitigkeitsvereinen ziemlich ungeändert geblieben, bei den Aktiengesellschaften hat dieser Anteil ununterbrochen zugenommen. Ebenso ist dieser Anteil auch bei der Volksversicherung der Aktiengesellschaften im Wachsen; auch bei den Gegenseitigkeitsvereinen zeigt sich dieses günstige Bild. 


\begin{tabular}{|c|c|c|c|c|c|c|c|c|c|c|}
\hline \multicolumn{11}{|c|}{ Am Gewinne waren in direkten Geschäfte beteiligt } \\
\hline \multirow{2}{*}{ Versicherungsart } & \multicolumn{5}{|c|}{ bei den Aktiengesellschaften } & \multicolumn{5}{|c|}{ bei den Gegenseitigkeitsvereinen } \\
\hline & 1906 & 1907 & 1908 & 1909 & 1910 & 1906 & 1907 & 1908 & 1909 & 1910 \\
\hline Große Versicherung & \multicolumn{10}{|c|}{ von je 1000 Policen } \\
\hline Versicherung auf den Todesfall ........ & 864 & 872 & $88 \mathrm{I}$ & 888 & 895 & 994 & 995 & 995 & 995 & 995 \\
\hline$n \quad n \quad$ Lebensfall $\ldots \ldots$ & 319 & 317 & $35 x$ & $34^{2}$ & 340 & 917 & 916 & 910 & 909 & 907 \\
\hline Rentenversicherung $\ldots \ldots \ldots \ldots \ldots \ldots$ & $?$ & $?$ & $?$ & $?$ & I & 8 Iо & 794 & 780 & 759 & 747 \\
\hline Kleine Versicherung & & & & & & & & & & \\
\hline Volksversicherung ........... & 688 & 693 & 701 & 708 & 714 & 926 & 932 & 941 & 945 & 945 \\
\hline Sonstige kleine Versicherung........... & 169 & 172 & 166 & 163 & 193 & 223 & 911 & 923 & 925 & 928 \\
\hline Große Versicherung & \multicolumn{10}{|c|}{ von je $1000 \mathscr{A}$ Versicherungssumme (Jahresrente) } \\
\hline Versicherung auf den Todesfall... & 918 & 923 & 929 & 932 & 935 & 998 & 998 & 999 & 999 & 999 \\
\hline$* \quad \quad \quad \quad$ Lebensfall $\ldots . .$. & 275 & 273 & 306 & 296 & 294 & 885 & 886 & $88 \mathrm{I}$ & $88 \mathrm{I}$ & $88 \mathrm{I}$ \\
\hline Rentenversicherung $\ldots \ldots \ldots \ldots$ & II & 12 & 13 & 14 & 16 & 664 & 650 & 639 & 626 & 619 \\
\hline Kleine Versicherung & & & & & & & & & & \\
\hline Volksversicherung. & 760 & 770 & 779 & 789 & 796 & 968 & 969 & 971 & 972 & 972 \\
\hline Sonstige kleine Versicherung. & 226 & 234 & 232 & 233 & 258 & 180 & 895 & 911 & 917 & 923 \\
\hline
\end{tabular}

Die Versicherungen mit Gewinnbeteiligung sind, wenn man von der kleinen Versicherung absieht, am häufigsten bei der Versicherung auf den Todesfall; hier nähern sich die Verhältniszahlen der Aktiengesellschaften denen der Gegenseitigkeitsvereine in bemerkenswerter Weise. Bei den Versicherungen auf den Lebensfall findet sich die Gewinnbeteiligung schon seltener, wenn auch der Unterschied bei den Gegenseitigkeitsvereinen sehr viel kleiner ist als bei den Aktiengesellschaften. Beachtenswert ist vielleicht, daß bei beiden Arten von Unternehmungen in der Todesfallversicherung die Policen der Zahl nach mit einem etwas geringeren Prozentsatz am Gewinn Anteil haben als die Versicherungssummen, während bei der Versicherung auf den Lebensfall das Entgegengesetzte der Fall ist. Die Versicherungen mit Anteil am Gewinne liegen also hinsichtlich der Höhe der Versicherung beim Todesfall über, beim Lebensfall unter dem Durchschnitte. Rentenversicherungen werden von den Aktiengesellschaften nur ausnahmsweise mit Gewinnbeteiligung der Versicherten abgeschlossen; von den Gegenseitigkeitsvereinen geschieht das naturgemäß viel häufiger; aber auch bei diesen Vereinen geht - soweit unsere Zahlen, bei denen Angaben über die zahlreichen Versicherungen steigender Renten fehlen, ein Urteil zulassen - die Gewinnbeteiligung bei der Rentenversicherung immer mehr zurück.

Die Versicherung auf den Invaliditätsfall wird nur von wenigen Gesellschaften als selbständige Versicherung betrieben; zumeist tritt sie als Zusatzversicherung zu der gemischten Kapitalversicherung auf, indem für den Fall der Invalidität Prämienfreiheit oder Prämienfreiheit in Verbindung mit Gewährung einer Rente (meist 5 oder 10 Prozent des versicherten Kapitals) bis zum Ablaufe der Versicherung ausbedungen wird. Hinsichtlich der hiermit verbundenen Unsicherheit in den Angaben über den Versicherungsbestand wird auf die Anmerkungen zu Tabelle I 6 S. 20 Bezug genommen.

Die Krankenversicherung eignet sich weniger als jede andere Versicherungsart f) Krankenzum Betriebe von einer Zentrale aus; sie erfordert, wenn sie ohne übergroße Gefälordung des Versicherers durchgeführt werden soll, eine weitgehende Kontrolle der An- 
sprüche erhebenden Versicherten, die ohne großen Verwaltungsapparat nicht zu ermöglichen ist. Tatsächlich befaßten sich im Jahre 19 IO denn auch nur wenige Gesellschaften mit der Krankenversicherung; von diesen Gesellschaften wickeln 2 nur das von andern Gesellschaften übernommene Geschäft ab, ohne selbst neue Versicherungen abzuschließen, vgl. Tabelle I 7 .

Versicherungseinrichtungen von Berufsvereinigungen

Von den für begrenzte Kreise bestimmten Versicherungsunternehmungen sind, wie schon oben erwähnt, in die Tabellen dieser Statistik nur die Versicherungseinrichtungen einiger größerer Berufsvereinigungen aufgenommen, obwohl diese Einrichtungen sehr erhebliche Unterschiede zeigen und sich deshalb nicht olne Zwang gemeinsam behandeln lassen.

Von den fraglichen 18 Unternehmungen betrieben 11 die Versicherung auf den Todesfall (meist Sterbekassen), davon zwei auch die Versicherung auf den Lebensfall und drei auch die Rentenversicherung (meist Pensionsversicherung); die übrigen sieben befaßten sich lediglich mit der Pensionsversicherung.

Im ganzen waren bei diesen Versicherungseinrichtungen Ende 19 10 in Kraft 307377 Policen über $167139882 \mathscr{M}$ auf den Todesfall (durchschnittlich $544 \mathscr{M}$ für eine Versicherung), 308 Policen über 1357918 $\mathscr{N}$ auf den Lebensfall (durchschnittlich $4409 \varkappa$ für eine Versicherung). In der Rentenversicherung liefen zu Ende des Berichtsjahrs 18723 Policen über $4990026 \varkappa$ Jahresrente (durchschnittlich $267 \mathscr{M}$ Jahresrente für eine Versicherung). Außerdem bestanden noch 3591 Versicherungen auf den Tolesfall mit veränderlicher Versicherungssumme und 36236 Pensionsversicherungen mit fast ausschließlich steigender Rente.

Bei der einfachen Verwaltung der meisten derartigen Versicherungsvereine ist es erklärlich, daß sie keine Versicherungen in Rückdeckung übernommen haben. Auch die in Rückdeckung gegebenen Beträge sind nicht eben beträchtlich.

Von den ausländischen Versicherungsunternehmungen, die im Jahre I 9 Io im Deutschen Reiche Versicherungsgeschäfte betrieben haben, soll hier nur der Bestand des deutschen Geschäfts erörtert werden, wie er in den Tabellen I 12 bis I 18 erscheint. Dabei wird von einer Trennung zwischen Aktiengesellschaften und Gegenseitigkeitsvereinen abgesehen.

Wie vorhin bei den deutschen allgemeinen Unternehmungen, betrachten wir $\%$ nächst den gesamten übernommenen Bestand und den davon für eigene Rechnung behaltenen Teil.

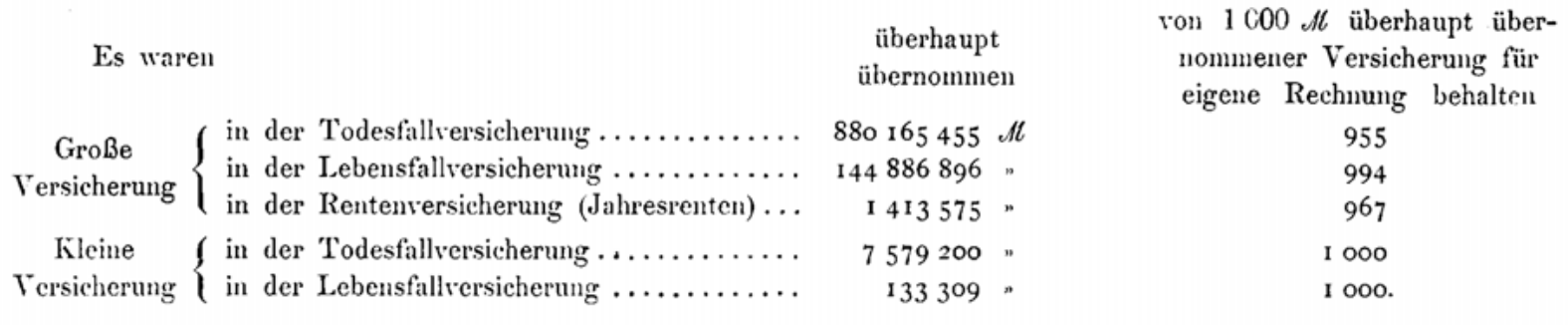

Danach haben die ausländischen Geselischaften nur in der großen Todesfallversicherung und in der Rentenversicherung einen nennenswerten Betrag in Rückdeckung gegeben. Aus der kleinen Versicherung haben sie bis Ende r 9 ro im Gegen- 
satze zu den deutschen Gesellschaften überhaupt nichts rückversichert, weil bei ihnen Quotenrückversicherungsverträge bis dahin nicht vorgekommen sind.

Uber das deutsche direkte Geschäft der ausländischen Unternehmungen gibt sodann folgende Zusammenstellung Auskunft.

\section{Deutsches direktes Geschäft der ausländischen Unternehmungen}

Versicherungssumme in $1000 \mathscr{A}$, Jahresrenten und Durchschnittsbeträge in $\mathscr{N}$

\begin{tabular}{|c|c|c|c|c|c|c|c|c|}
\hline & \multicolumn{6}{|c|}{ Großc Versicherung } & \multirow{2}{*}{\multicolumn{2}{|c|}{$\begin{array}{l}\text { Volks- } \\
\text { versicherung }\end{array}$}} \\
\hline & \multicolumn{2}{|c|}{ auf den Todesfall } & \multicolumn{2}{|c|}{ auf den Lebensfall } & \multicolumn{2}{|c|}{ Rentenversicherung } & & \\
\hline & 1905 & 1910 & 1905 & 1910 & 1905 & 1910 & 1905 & 1910 \\
\hline Zahl der Policen $\ldots \ldots \ldots \ldots \ldots$ & 123969 & $13^{8807}$ & 46639 & 77083 & 1839 & 2132 & 1 I 993 & 28531 \\
\hline Versicherungssummen (Jahresrenten) & 799215 & $85^{8} 776$ & 123955 & 144810 & 1274013 & I 388974 & 4006 & 7713 \\
\hline Durchschnittsbeträge $\ldots \ldots \ldots \ldots \ldots$ & 6447 & 6187 & 2658 & I 879 & 693 & 651 & 334 & 270 \\
\hline
\end{tabular}

Die Durchschnittsbeträge sind bei den ausländischen (iesellschaften merklich hölıer als bei den deutschen Anstalten, doch rührt das, soweit es sich um die große Kapitalversicherung handelt, daher, daß einige wenige Gesellschaften besonders hohe Beträge übernommen haben; es handelt sich nicht um eine Eigentümlichkeit aller ausländischen Unternehmungen.

Uber das Verhältnis der mit Gewinnbeteiligung der Versicherten abgeschlossenen Verträge zu den überhaupt abgeschlossenen Verträgen und über die Entwickelung dieses Verhältnisses in den letzten fünf Jahren ergeben sich bei Beschränkung auf das direkte Geschäft folgende Zahlen:

Im deutschen dirckten Geschäfte der ausländischen Unternehmungen waren am Gewinne beteiligt:

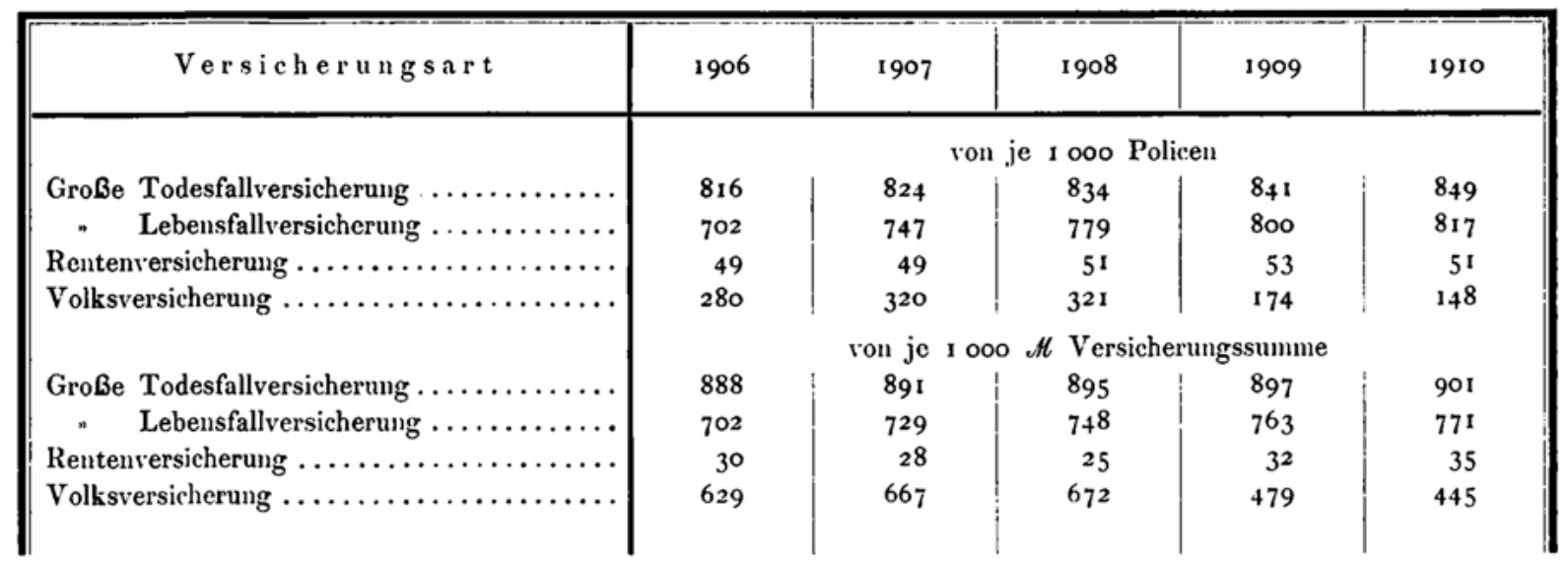

Wie bei den deutschen Gesellschaften, ist auch bei den ausländischen Unternehmungen die Gewinnbeteiligung der Versicherten bei der Todesfallversicherung am häufigsten. Bezüglich der Versicherungssumme ist die Gewinnbeteiligung der Versicherten bei den ausländischen Anstalten geringer als bei den deutschen Aktiengesellschaften. 
In der Volksversicherung schließen die ausländischen Gesellschaften bei uns verhältnismäßig sehr viel weniger Verträge mit Gewinnbeteiligung ab als die deutschen Unternehmungen.

\section{Die Bewegung im Versicherungsbestand im Jahre 1910}

Deutsche allgemeine Unternelmungen

Die Bewegung in dem Versicherungsbestande der deutschen Unternehmungen, die allen Kreisen der Bevölkerung offenstelten, ist in den Tabellen I 19 bis I 23 ausführlich behandelt. Dabei hat aber, wie schon oben bemerkt ist, die Trennung zwischen großer und kleiner Versicherung nicht so vollständig durchgeführt werden können wie bei der Beschreibung des Bestandes am Ende des Berichtsjahrs; die Bewegung der Sterbegeldversicherung war bei einigen Unternehmungen nicht aus der Bewegung der großen Versicherung auszuscheiden.

Die folgende Zusammenstellung gibt einen Uberblick über die Bewegung der wichtigsten Versicherungsarten in dem gesamten Geschäfte der großen deutschen Unternehmungen; dabei ist bei der Volksversicherung und bei der sonstigen kleinen Versicherung die Todesfallversicherung mit der Lebensfallversicherung zusammengefaßt. In Betracht gezogen sind nur die selbst abgeschlossenen Versicherungen.

Uberall zeigt sich ein erfreulicher Fortschritt; nur die Versicherungen auf den Lebensfall gehen zurück sowohl bei den Aktiengesellschaften wie bei den Gegenseitigkeitsvereinen.

Bewegung im Versicherungsbestande der deutschen Unternehmungen

\begin{tabular}{|c|c|c|c|c|c|c|c|c|c|c|}
\hline & \multicolumn{2}{|c|}{$\begin{array}{l}\text { Versicherung } \\
\text { auf } \\
\text { den Todesfall }\end{array}$} & \multicolumn{2}{|c|}{$\begin{array}{c}\text { Versicherung } \\
\text { auf } \\
\text { den Lebensfall }\end{array}$} & \multicolumn{2}{|c|}{$\begin{array}{c}\text { Renten- } \\
\text { versicherung }\end{array}$} & \multicolumn{2}{|c|}{$\begin{array}{c}\text { Volks- } \\
\text { versicherung }\end{array}$} & \multicolumn{2}{|c|}{$\begin{array}{c}\text { Sonstige kleine } \\
\text { Versicherung }\end{array}$} \\
\hline & Policen & $1000 \mathscr{H}$ & Policen & 1000 edt & Policen & $1000 \mathscr{A}$ & Policen & $1000 \mathscr{H}$ & Policen & $1000 . / t$ \\
\hline \multicolumn{11}{|c|}{ Aktiengesellschaften } \\
\hline & \multicolumn{2}{|c|}{$\begin{array}{l}27 \text { Gesell- } \\
\text { schaften }\end{array}$} & \multicolumn{2}{|c|}{$\begin{array}{l}25 \text { Gesell- } \\
\text { schaften }\end{array}$} & \multicolumn{2}{|c|}{$\begin{array}{l}23 \text { Gesell- } \\
\text { schaften }\end{array}$} & \multicolumn{2}{|c|}{$\begin{array}{l}11 \text { Gesell- } \\
\text { schaften }\end{array}$} & \multicolumn{2}{|c|}{$\begin{array}{l}10 \text { Gesell- } \\
\text { schaften }\end{array}$} \\
\hline Bestand Ende r 909 & I 317030 & 5517982 & 254930 & 435064 & 35811 & 18906 & 6494407 & 1213217 & 63225 & 26509 \\
\hline Zugang....... & I69845 & 788468 & I5 409 & 28544 & $33^{87}$ & 2073 & I I 10697 & 249479 & 10 898 & 4966 \\
\hline Abgang...$\ldots \ldots$ & 80654 & 334384 & 21946 & 40419 & I 900 & I 055 & $75626 \mathrm{I}$ & I 66464 & 6912 & 3129 \\
\hline Reiner Zugang... & 89191 & 454084 & -6537 & $-\begin{array}{ll}11 & 875\end{array} \mid$ & 1487 & 1018 & 354436 & 83 OI 5 & 3986 & I 837 \\
\hline Bestand Ende I910 & I 40622 I & 5972066 & 248393 & 423189 & 37298 & 19924 & 6848843 & I 296232 & 67211 & 28346 \\
\hline \multicolumn{11}{|c|}{ Gegenseitigkeitsvereine } \\
\hline & \multicolumn{2}{|c|}{14 Vereine } & \multicolumn{2}{|c|}{13 Vereine } & \multicolumn{2}{|c|}{10 Vereine } & \multicolumn{2}{|c|}{2 Vereine } & \multicolumn{2}{|c|}{7 Vereine } \\
\hline Bestand Ende 1909 & 836254 & 4591406 & 255822 & $|400917|$ & 21833 & 5460 & 343558 & 57600 & 303900 & $\begin{array}{lll}\text { I } & 5 & 243\end{array}$ \\
\hline Zugang...$\ldots \ldots$ & 73850 & $4309 \mathrm{I} 3$ & 16097 & 28658 & 1115 & $45^{8}$ & 127236 & 22766 & 21767 & 15912 \\
\hline Abgang $\ldots \ldots \ldots$ & $3^{2} 43^{8}$ & 170145 & 2I 764 & 33540 & I 154 & 225 & 94990 & 16406 & II I 80 & 5832 \\
\hline Reiner Zugang ... & 41412 & 260768 & -5667 & -4882 & -39 & 233 & 32246 & 6360 & I0 587 & 10080 \\
\hline Bestand Ende 1910 & 877666 & 4852 I 74 & 250 I 55 & $|396035|$ & 2 I 794 & 5693 & 375804 & 63960 & $|314487|$ & 125323 \\
\hline \multicolumn{11}{|c|}{ Sämtliche Unternehmungen } \\
\hline & \multicolumn{2}{|c|}{$\begin{array}{c}41 \text { Unter- } \\
\text { nehmungen }\end{array}$} & \multicolumn{2}{|c|}{$\begin{array}{l}38 \text { Unter- } \\
\text { nehmungen }\end{array}$} & \multicolumn{2}{|c|}{$\begin{array}{l}33 \text { Unter- } \\
\text { nehmungen }\end{array}$} & \multicolumn{2}{|c|}{$\begin{array}{l}13 \text { Unter- } \\
\text { nehmungen }\end{array}$} & \multicolumn{2}{|c|}{$\begin{array}{l}17 \text { Unter- } \\
\text { nehmungen }\end{array}$} \\
\hline Bestand Ende 1909 & $\begin{array}{llll}2 & 1 & 53 & 28\end{array}$ & I0 109388 & $51075^{2}$ & $83598 \mathrm{I} \mid$ & 57644 & 24366 & 6837965 & I 270817 & $|367 \quad 125|$ & $14175^{\prime}$ \\
\hline Zugang ..... & 243695 & I 2 I $93^{81}$ & 31506 & 57202 & 4502 & 2531 & I 237933 & 272245 & 32665 & 20878 \\
\hline Abgang $\ldots \ldots \ldots$ & I1 3092 & 504529 & 43710 & 73959 & $3 \circ 54$ & I 280 & 851251 & I 82870 & 18092 & $896 \mathbf{1}$ \\
\hline Reiner Zugang ... & 130603 & 714852 & -12204 & -16757 & I 448 & $125 \mathrm{I}$ & 386682 & 89375 & I 4573 & 11917 \\
\hline nd Ende r9io & $\begin{array}{llll}2 & 28 & 88 & 887\end{array}$ & 10824240 & 498548 & $\mid \begin{array}{lll}81924 & 22\end{array}$ & 59092 & 25617 & $|7224647|$ & $\left|\begin{array}{lll}1 & 360 & 192\end{array}\right|$ & 381698 & $\mid$ I53669| \\
\hline
\end{tabular}


Wegen der großen Bedeutung, die den verschiedenen Ursachen des Abgangs von Versicherungen zukommt, sind in unseren Tabellen für die Hauptversicherungsarten nicht nur die absoluten Zahlen für die Häufigkeit der einzelnen Ursachen angegeben, sondern es ist auch berechnet, wieviel bei den selbst abgeschlossenen Versicherungen von 1000 Policen oder von $1000 \mathscr{M}$ Versicherungssumme des durchschnittlichen Bestandes durch Tod, wieviel durch Ablauf usw. in Wegfall gekommen sind. An dieser Stelle wird es genügen, die Ursachen des Wegfalls in drei Gruppen zusammenzufassen: ordentlicher Abgang (Abgang durch Tod oder Ablauf), Storno mit Vergütung (Abgang durch Rückkauf, Reduktion, Umwandlung oder Ubertragung) und Storno ohne Vergütung (Abgang durch Verfall, Verzicht, Nichteinlösung der Police).

\begin{tabular}{|c|c|c|c|c|c|c|}
\hline \multirow[b]{2}{*}{ Versicherung auf den Todesfall } & \multicolumn{2}{|c|}{ ordentlicher Abgang } & \multicolumn{2}{|c|}{ Storno mit Vergütung } & \multicolumn{2}{|c|}{ Storno ohne Vergütung } \\
\hline & Policen & Summen & Policen & Summen & Policen & Summen \\
\hline Aktiengesellschaften ....... & 18 & 18 & 12 & 14 & 29 & 26 \\
\hline Gegenseitigkeitsvereine ...... & 19 & 19 & 8 & 9 & II & 8 \\
\hline Versicherung auf den Lebensfall & & & & & & \\
\hline Aktiengesellschaften..$\ldots \ldots$ & $5^{2}$ & 49 & 16 & 24 & 19 & 21 \\
\hline Gegenseitigkeitsvereine ...... & 66 & 57 & 7 & 11 & I 2 & 16 \\
\hline Rentenversicherung & & & & & & \\
\hline Aktiengesellschaften...$\ldots \ldots$ & 43 & 42 & 5 & 9 & 4 & 3 \\
\hline Gegenseitigkeitsrereine ...... & 47 & 36 & 6 & 3 & $\mathbf{I}$ & $\mathbf{I}$ \\
\hline Volksversicherung & & & & & & \\
\hline Aktiengesellschaften..$\ldots \ldots$. & 39 & 31 & 2 & 13 & 73 & 87 \\
\hline Gegenseitigkeitsvereine ..... & 34 & 24 & 15 & 19 & 216 & 227 \\
\hline
\end{tabular}

An diesen Zahlen ist $u$. a. beachtenswert, daß bei der Volksversicherung der Aktiengesellschaften ein höherer Satz der Versicherungssummen als der Policenzahl durch Storno ohne Entschädigung ausscheidet, und daß bei der großen Todesfallversicherung das Entgegengesetzte stattfindet. Bei der Volksversicherung der Aktiengesellschaften verfallen danach mehr verhältnismäßig hohe, bei der großen Versicherung mehr verhältnismäßig niedrige Versicherungen.

Die außerordentlich kleinen Zahlen für den vorzeitigen Abgang bei der Rentenversicherung finden ihre einfache Erklärung darin, daß es sich hier in der überwiegenden Zahl der Fälle um sofort beginnende Renten gegen einmalige Prämien handelt; ein vorzeitiger Abgang kann aber nur bei den verhältnismäßig seltenen Versicherungen von aufgeschobenen Renten gegen Jahresprämien vorkommen.

Wenn man bei den Versicherungseinrichtungen der größeren Berufsvereinigungen (Pensionskassen und Sterbekassen) diejenigen Kassen außer Betracht läßt, bei welchen die Versicherungssummen und Jahresrenten als veränderlich nicht angegeben werden können, erhält man folgendes Bild von der Bestandsänderung im Berichtsjahre:

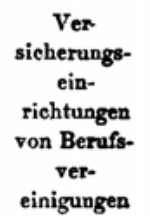

\begin{tabular}{|c|c|c|c|c|}
\hline & \multicolumn{2}{|c|}{$\begin{array}{c}\text { Kapitalversicherung } \\
10 \text { Vereine }\end{array}$} & \multicolumn{2}{|c|}{$\begin{array}{c}\text { Rentenversicherung } \\
5 \text { Vereine }\end{array}$} \\
\hline & Policen & $1000 \mathscr{N}$ & Policen & $1000 \mathscr{N}$ Rente \\
\hline Bestand Ende $1909 \ldots \ldots \ldots \ldots \ldots$ & 290462 & $15535^{\circ}$ & 18572 & 4855 \\
\hline Zugang $\ldots \ldots \ldots \ldots \ldots \ldots \ldots \ldots \ldots$ & 32712 & 18311 & 547 & 240 \\
\hline Abgang $\ldots \ldots \ldots \ldots \ldots \ldots \ldots \ldots \ldots$ & 15489 & 5163 & 396 & 105 \\
\hline 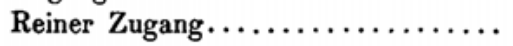 & 17223 & 13148 & $15 x$ & 135 \\
\hline 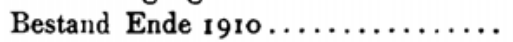 & 307685 & 168498 & 18723 & 4990 \\
\hline
\end{tabular}


Auch bei diesen Anstalten zeigt sich also eine Zunahme der Policenzahl wie auch der Versicherungssummen und der Jahresrenten.

Die Bewegung in dem deutschen Versicherungsbestande der ausländischen Lebensversicherungsunternehmungen ist in den Tabellen I 25 bis I 28 ausführlich dargestellt. Aus diesen Tabellen ist folgende Ubersicht hergeleitet:

Bewegung im deutschen Versicherungsbestand der ausländischen Gesellschaften

\begin{tabular}{|c|c|c|c|c|c|c|c|c|}
\hline & \multicolumn{2}{|c|}{$\begin{array}{l}\text { Versicherung auf } \\
\text { den Todesfall } \\
21 \text { Gesellschaften }\end{array}$} & \multicolumn{2}{|c|}{$\begin{array}{l}\text { Versicherung auf } \\
\text { den Lebensfall } \\
21 \text { Gesellschaften }\end{array}$} & \multicolumn{2}{|c|}{$\begin{array}{c}\text { Renten- } \\
\text { versicherung } \\
18 \text { Gesellschaften }\end{array}$} & \multicolumn{2}{|c|}{$\begin{array}{c}\text { Volks- } \\
\text { versicherung } \\
3 \text { Gesellschaften }\end{array}$} \\
\hline Bestand Ende 1909 .. & I 35053 & 837495 & 73003 & 143487 & 2068 & I 344 & 23330 & 6595 \\
\hline 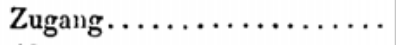 & I 3030 & 84216 & 10405 & I 4524 & 146 & 99 & 18943 & 3806 \\
\hline Abgang $\ldots \ldots \ldots \ldots$ & 9034 & 62898 & 6325 & 13201 & 82 & 54 & 13984 & 2725 \\
\hline Reincr Zugang...$\ldots \ldots$ & 3996 & 21318 & 4080 & I 323 & 64 & 45 & 4959 & I $08 \mathrm{r}$ \\
\hline Bestand Ende 1910... & 139049 & $8588_{13}$ & 77083 & 144810 & 2132 & I 389 & 28289 & 7676 \\
\hline
\end{tabular}

Danach hat das deutsche Geschäft der ausländischen Gesellschaften im Jahre 1910 überall Fortschritte gemacht.

Uber die Häufigkeit der verschiedenen Arten des Abganges seien hicr die folgenden Zahlen aufgeführt, die den oben für die deutschen Unternehmungen angegebenen Zahlen entsprechen.

Abgang in Tausendsteln des durchschnittlichen Bestandes

\begin{tabular}{|c|c|c|c|c|c|}
\hline ordentli & Abgang & Storno mit & Vergütung & Storno ohne & Vergütung \\
\hline Policen & Summen & Policen & Summen & Policen & Summen \\
\hline 26 & 30 & I 6 & 22 & 25 & $2 \mathrm{I}$ \\
\hline 28 & 47 & 22 & 20 & 34 & 25 \\
\hline 12 & IO & I I & 17 & 539 & $36 \mathrm{r}$ \\
\hline
\end{tabular}

Ein Vergleich der Zahlen für den ordentlichen Abgang mit den für die früheren Jahre geltenden Zahlen läßt bei der großen Todesfallversicherung eine langsame, aber fast ununterbrochene Zunahme erkennen; 1902 betrug der Abgang bei den Policen nur 21 und bei der Versicherungssumme nur 20 Tausendstel des mittleren Jahresbestandes.

\section{Die Betriebsrechnung für 1910}

allgemeines Die Gewinn- und Verlustrechnung, wie sie in den Tabellen I 29,32, 34 und 36 gegeben ist, unterscheidet sich von den Aufstellungen, welche die Unternehmungen nach dem Formular L 1 der Rechnungsvorschriften dem Aufsichtsamt einzureichen haben, dadurch, daß die veränderlichen Fonds (Prämienreserve, Prämienüberträge, Reserven für schwebende Versicherungsfälle, Gewinnreserven und sonstige Reserven) nicht mit ihren Bestïnden zu Anfang des Jahres in Einnahme und mit ihren Werten am Ende des Jahres in Ausgabe gestellt sind, sondern daß nur die durch den Jahresbetrieb 
hervorgerufenen Änderungen nachgewiesen sind; denn nur diese Änderungen beeinflussen das Ergebnis der Jahresrechnung; außerdem wird die Rechnung durchsichtiger. Der Stand der Fonds am Ende des Jahres findet sich in der Bilanz. Sodann ist der Vortrag aus dem Überschusse des Vorjahrs hier fortgelassen worden, um das Ergebnis des Geschäftsbetriebs lediglich aus dem Berichtsjahre zu erhalten.

Die Betriebsrechnung bezieht sich auf das ganze (deutsche und ausländische) Lebensversicherungsgeschäft der Unternehmungen. Für die Volksversicherung ist bei den deutschen Gesellschaften eine besondere Betriebsrechnung (Tabelle I 32) auf'gestellt; die sonstige kleine Versicherung und bei den ausländischen Gesellschaften auch die Volksversicherung ist mit dem Geschäft in der großen Versicherung zusammen verrechnet (Tabelle I 29 und 36). Auch sind beim "Atlas ", bei der "Wilhelma " und bei der "Bayer. V. B." die geringen Einnahmen und Ausgaben der Kautionsversicherung in der allgemeinen Betriebsrechnung für das gesamte Lebensversicherungsgeschäft enthalten (vgl. die Anmerkungen 4, 5 und 6 zu Tabelle I 30 S. 93).

Die in den Übersichten über die Gewinn- und Verlustrechnung nur im Ganzen eingestellten Prämien und Vergütungen der Rückversicherer einerseits und die Zahlungen für selbst abgeschlossene und für in Rückdeckung übernommene Versicherungen sowie die Rückversicherungsprämien anderseits sind in den Tabellen I 30,33 und 35 erläutert.

In der Lebensversicherung olne Volksversicherung haben betragen:

\begin{tabular}{|c|c|c|c|}
\hline & $\begin{array}{l}\text { bei der Gesamtheit } \\
\text { der Unternehmungen }\end{array}$ & $\begin{array}{l}\text { bei } 26 \text { Abtien- } \\
\text { gescllschaften }\end{array}$ & $\begin{array}{c}\text { bei } 16 \text { Gegen- } \\
\text { seitigkeitsvereinen }\end{array}$ \\
\hline die Summe der Einnahmen ......... & 714437725 th & $407318616 \mathscr{M}$ & $307+19109 \mathrm{All}$ \\
\hline die Summe der Ausgaben .......... & $595037690 "$ & $346380354 \cdots$ & $248657336 \cdots$ \\
\hline der Jahresüberschuß ... & $119400035 \mathscr{A}$ & $60938262 \mathscr{A l}$ & $58461773 \mathscr{A l}$ \\
\hline
\end{tabular}

Das Geschäftsjahr i9 10 hat allen 26 Aktiengesellschaften in der großen Lebensversicherung einen Überschuß von zusammen $60938262 \mathscr{M}$ gebracht; bei den 16 Gegenseitigkeitsvereinen ist ein Gewinn von zusammen $58461773 \mathscr{M}$ erzielt worden. Von dem durch die Bilanz ausgewiesenen Gewinn und dessen Verteilung wird unten gesprochen werden.

Für die Volksversicherung ergeben sich folgende Zahlen:

\begin{tabular}{|c|c|c|c|}
\hline & Gesamtheit & 11 Aktiengesellschaften & $\begin{array}{c}2 \text { Gegenseitigkeits- } \\
\text { vereine }\end{array}$ \\
\hline Summe der Einnahmen....... & $\mathrm{I} 29692004 \mathscr{A C}$ & 12344 I $770 \mathscr{M}$ & $6250234 \mathscr{N}$ \\
\hline Summe der Ausgaben......... & $108747372 n$ & $103670389 n$ & $5076983 \cdots$ \\
\hline Jahresüberschuß $\ldots$ & $20944632 \pi$ & $19771381 \mathscr{H}$ & $1173251 \mathscr{A}$ \\
\hline
\end{tabular}

Von den 11 Aktiengesellschaften, deren Betriebsrechnung für das Volksversicherungsgeschäft in Tabelle I 32 aufgestellt ist, hat im Jahre 1910 eine Gesellschaft mit einem Verluste von $19840 \mathscr{N}$ gearbeitet; an dem Gewinne von $19791221 \mathscr{N}$ sind 10 Anstalten beteiligt. Die beiden Gegenseitigkeitsvereine haben einen Uberschuß von zusammen $1173251 \mathscr{N}$ erzielt.

Für eingetretene Versicherungsfälle des Geschäftsjahrs aus selbst abgeschlossenen Versicherungen wurden im Berichtsjahr in der großen Versicherung von der Gesamtheit der Unternehmungen 94,4 Prozent der fällig gewordenen Summen ausbézahlt und 
5,6 Prozent zurückgestellt; im besonderen von den Aktiengesellschaften 95,6 und 4,4 Prozent, von den Gegenseitigkeitsvereinen 93,1 und 6,9 Prozent. In der Volksversicherung wurden von der Gesamtheit der Unternehmungen 97,39 Prozent aller fällig gewordenen Versicherungssummen gezahlt und 2,61 Prozent zurückgestellt; im besonderen bei den Aktiengesellschaften 97,44 und 2,56 Prozent, bei den Gegenseitigkeitsvereinen 95,98 und 4,02 Prozent. Bei Vergleichen mit den früheren Jahren ist zu beachten, daß die Zahlungen aus Versicherungsfällen bei der "Victoria " seit 1909 die mit den Versicherungssummen ausgezahlten Gewinnanteile nicht mehr enthalten; diese Beträge sind vielmehr bei den an die Versicherten ausgezahlten Gewinnanteilen verrechnet.

Ver-

siclierungs-

einrichtungen von

Berufsvereinigungen

Über die Gewinn- und Verlustrechnung der in diese Statistik aufgenommenen Versicherungseinrichtungen der größeren Berufsvereinigungen geben die Tabellen I 34 und I 35 Aufschluß.

Daraus sind folgende Zahlen zu entnehmen:

18 Versicherungseinrichtungen
Summe der Einnahmen $\ldots \ldots \ldots \ldots \ldots$

Einen Fehlbetrag hat im Berichtsjahre nur eine dieser Anstalten mit $10690 \mathscr{M}$ ausgewiesen; die übrigen 17 Anstalten erzielten einen Überschuß von zusammen $2448247 \mathscr{M}$.

Zur Beurteilung des Geschäftsbetriebs derjenigen ausländischen Lebensversicherungsgesellschaften, über welche diese Statistik berichtet, ist in Tabelle I 36 die Gewinnund Verlustrechnung für das ganze inländische und ausländische Geschäft möglichst nach denselben Grundsätzen dargestellt, die bei den deutschen Gesellschaften zur Anwendung gekommen sind. Daneben sind aber in Tabelle I 37 die Einnahmen und Ausgaben der ausländischen Gesellschaften allein aus dem deutschen Geschäfte zusammengestellt. Es haben betragen die Einnahmen an Prämien im deutschen Geschäft insgesamt $43987140 \mathscr{M}$. Davon kommen $43174244 \mathscr{M}$ oder 98,2 Prozent auf selbst abgeschlossene Versicherungen und $812896 \mathscr{M}$ oder 1,8 Prozent auf die in Rückdeckung übernommenen Versicherungen. Für Versicherungsleistungen einschließlich der Zahlungen für vorzeitig aufgelöste Versicherungen waren bereitzustellen insgesamt $36948572 \mathscr{M}$, davon $36639120 \mathscr{M}$ oder 99,2 Prozent für selbst abgeschlossene Versicherungen und $309452 \mathscr{M}$ oder 0,8 Prozent für in Rückdeckung übernommene Versicherungen.

\section{Die Bilanz für den Schluß des Jahres 1910}

Deutsche allgemeine Unternehmungen
Eine Bilanz ist in dieser Statistik grundsätzlich für das Gesamtgeschäft einer jeden Gesellschaft aufgestellt und bei derjenigen Gruppe nachgewiesen, zu der das Unternehmen seinem Hauptbetriebe nach gehört.

Dementsprechend sind in Tabelle I 38 die Bilanzen für 26 Aktiengesellschaften und 15 Gegenseitigkeitsvereine aufgeführt; die Gesamtbilanz schließt für Ende I 9 Io bei den Aktiengesellschaften mit $2969920613 \mathscr{N}$ und bei den Gegenseitigkeitsvereinen mit $2025060642 \mathscr{N}$ ab; als Hauptposten sind folgende Beträge zu verzeichnen: 
Alitiva

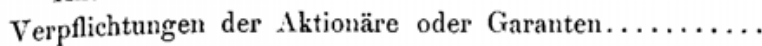

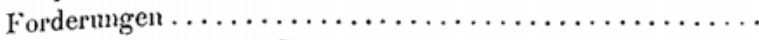

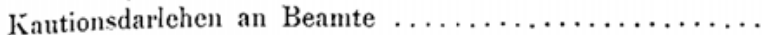

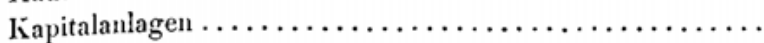

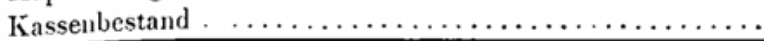

Passiva

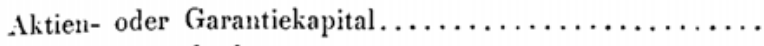

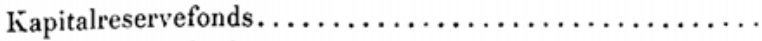

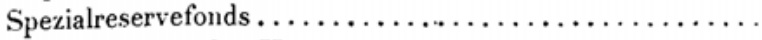

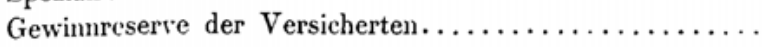

Guthaben anderer Versicherungsunternehmungen ........

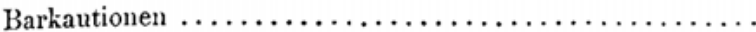

Prämienreserven und Prämienüberträge

Schadenreserve
26 Aktiengesellschaften

I 21325551

142471074 n 396568 "

2694129087 "

3780087 "

$$
\begin{array}{r}
162815697 d \\
15112829 " \\
69416836 " \\
221876472 " \\
5306354 " \\
763420 " \\
2362366280 " \\
17913007 "
\end{array}
$$

15 Gegenseitigkeitsvereine $433950 \mathscr{H}$

\begin{tabular}{|c|c|c|c|c|c|c|c|c|}
\hline \multirow[b]{2}{*}{ Grundbesitz $\ldots \ldots \ldots \ldots \ldots \ldots \ldots \ldots \ldots$} & \multicolumn{4}{|c|}{26 Aktiengesellschaften } & \multicolumn{4}{|c|}{15 Gegenseitigkeitsvereine } \\
\hline & 71662262 & th oder & 2,7 & $\%$ & 15859946 & $\mathscr{A l}$ oder & 0,8 & $\%$ \\
\hline Hypotheken.$\ldots \ldots \ldots \ldots \ldots \ldots \ldots \ldots$ & $230967715 \mathrm{r}$ & $n \quad n$ & 85,7 & $"$ & I 604208465 & $n \quad \cdot$ & 82,6 & $"$ \\
\hline Darlehen an öffentliche Körperschaften & $45494 \quad 145$ & n & $\mathrm{I}, 7$ & $n$ & 125368526 & $"$ & 6,5 & " \\
\hline Policedarlehen $\ldots \ldots \ldots \ldots \ldots \ldots \ldots$ & I96 58I 68I & " & 7,3 & " & I 38653109 & n & 7,2 & $n$ \\
\hline Darlehen auf Wertpapiere........... & 86500 & $n$ & 0,0 & $n$ & I 594254 & " & $0, \mathbf{I}$ & $"$ \\
\hline 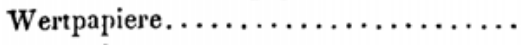 & $6864155^{8}$ & . & 2,5 & $\cdot$ & 51136502 & " & 2,6 & $n$ \\
\hline Wechsel $\ldots \ldots \ldots \ldots \ldots \ldots \ldots \ldots \ldots \ldots$ & I 985790 & " & $0, \mathbf{I}$ & $n$ & 4537288 & • & 0,2 & $\cdot$ \\
\hline Sonstige Anlagen $\ldots \ldots \ldots \ldots \ldots \ldots$ & - & & - & & - & & 一 & \\
\hline
\end{tabular}

75695453 "

I 387382 ”

1941358090 "

$251117 \mathrm{I}$

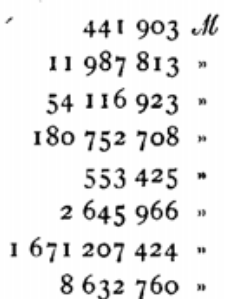

Die hier aufgeführten Kapitalanlagen verteilen sich auf folgende Wertarten (Tabelle I 39):

Vergleicht man diese Zahlen mit denen des Vorjahrs, so erhält man folgendes Bild: Bei den Aktiengesellschaften haben zugenommen der Wert des Grundbesitzes um $2748798 \mathscr{M}$, der Hypothekenbestand um $191159956 \mathscr{M}$, die Darlehen an öffentliche Körperschaften um $7274798 \mathscr{M}$, die Policedarlehen um $13555320 \mathscr{M}$, die Wertpapiere um $1478571 \mathscr{M}$ und der Bestand an Wechseln um $429267 \mathscr{N}$; abgenommen haben dagegen die Darlehen auf Wertpapiere um 554700 $\mathscr{N}$. Bei den Gegenseitigkeitsvereinen liaben zugenommen der Hypothekenbestand um $59987777 \mathscr{M}$, die Darlehen an öffentliche Körperschaften um $17282067 \mathscr{N}$ und die Policedarlehen um 4872884 $\mathscr{N}$; dagegen hat der Wert des Grundbesitzes um $1271941 \mathscr{M}$, die Darlehen auf Wertpapiere um $400925 \mathscr{N}$, die Wertpapiere um $2743787 \mathscr{N}$ und der Bestand an Wechseln um $1379855 \mathscr{M}$ abgenommen. Dem Kapitalmarkte haben die allgemeinen deutschen Lebensversicherungsunternehmungen zugeführt: 1,48 Millionen Mark für Grundbesitz, 251,15 Millionen für Hypotheken und 24,56 Millionen für Darlehen an öffentliche Körperschaften; entzogen sind dem Kapitalmarkte für Wechsel und für Darlehen auf Wertpapiere je 1 Million und nahezu 1,4 Millionen für Wertpapiere (nach dem Nennwerte).

Die Prämienreserven und die Prämienüberträge sind in Tabelle I 40 nach Versicherungsarten getrennt angegeben. Hierzu bedarf es indessen noch folgender Erläuterungen. Nach $§ 58$ des Versicherungsaufsichtsgesetzes soll bei Rückversicherungen die Prämienreserve von dem rückversicherten Unternehmen berechnet, aufbewahrt und verwaltet werden. Die Rechnungsvorschriften bestimmen, daß als Prämienreserve in der Gewinnund Verlustrechnung und in der Bilanz nur die in eigener Verwaltung befindlichen Beträge geführt werden sollen. Für alle bis zum Inkrafttreten des Gesetzes abgeschlossenen Versicherungen sollen die Prämienreserven unter Berücksichtigung der geltenden Rück- 
versicherungsverträge eingestellt werden. Bei den nach dem Inkrafttreten des Gesetzes abgeschlossenen Versicherungen sollen die Prämienreserven für alle selbst abgeschlossenen Versicherungen einschließlich der in Rückdeckung gegebenen Summen unter der Position Prämienreserve nachgewiesen werden; für die in Rückdeckung übernommenen Versicherungen aber, soweit die Prämienreserven abzugeben sind, sollen diese Prämienreserven in der Gewinn- und Verlustrechnung bei den Vergütungen für in Rückdeckung übernommene Versicherungen verrechnet werden, also auch in der Bilanz nicht bei den Prämienreserven erscheinen. Die Gesellschaften verfahren bei der Behandlung der Prämienreserven nicht gleichmäßig. Ein Teil der Anstalten läßt die Prämienreserven in fremder Verwaltung ganz aus der Rechnung fort, wälırend die übrigen Gesellschaften diese Posten bei anderen Positionen behandeln. Da das Formular I 3 der Rechnungsvorschriften die Nittel bietet, den Betrag der bei Rückversicherungen abgegebenen Reserven festzustellen, so sind diese Beträge in unsern Statistiken überall ausgeschieden. Dieses Verfahren entspricht nicht nur den Rechnungsvorschriften, sondern verhindert auch, daß die Reserven für solche Versicherungen, die von einer unter Reichsaufsicht stehenden Gesellschaft bei einer ebensolchen Gescllschaft in Rückdeckung gegeben sind, mehrfach aufgeführt werden.

Die Trennung der Prämienreserven und der Prämienüberträge erfolgt bei den verschiedenen Anstalten und bei verschiedenen Versicherungsarten nicht nach denselben Grundsätzen, so daß die als Prämienreserven oder Prämienüberträge angegebenen Summen weder die reinen Prämienreserven noch die reinen Utberträge darstellen. Prämienreserven und Prämienüberträge sind deshalb zusammengefaßt worden.

In Tabelle I 40 sind für alle deutschen Aktiengesellschaften Prämienreserven und Prämienüberträge in eigener Verwaltung in Höhe von 2426,181 Millionen Mark nachgewiesen. Davon kommen 2338,195 Millionen Mark auf die Lebensversicherung und 87,986 Millionen Mark auf andere Versicherungszweige. Bei den Gegenseitigkeitsvereinen gehören die in der Tabelle aufgeführten Beträge von zusammen 1701,709 Millionen Mark vollständig der Lebensversicherung an. Die gesamte Prämienreserve für Lebensund Krankenversicherung stellt sich danach für alle deutschen allgemeinen Unternehmungen auf 4039,904 Millionen Mark, wovon 5 $\mathbf{\imath}, 0$ Prozent auf die Aktiengesellschaften und 42,1 Prozent auf die großen Gegenseitigkeitsvereine entfallen. In welchem Maße die einzelnen Versicherungsarten beteiligt sind, ist aus nachstehender Ubersicht zu entnehmen:

\begin{tabular}{|c|c|c|c|c|c|c|}
\hline \multirow{3}{*}{ Versicherungsart } & \multicolumn{6}{|c|}{ Prämienreserven und Prämienüberträge } \\
\hline & \multicolumn{2}{|c|}{$\begin{array}{l}\text { bei der Gesamtheit } \\
\text { der Unternehmungen }\end{array}$} & \multicolumn{2}{|c|}{$\begin{array}{l}\text { bei den Aktien- } \\
\text { gesellschaften }\end{array}$} & \multicolumn{2}{|c|}{$\begin{array}{l}\text { bei den Gegen- } \\
\text { seitigkeitsvereinen }\end{array}$} \\
\hline & $1000 \mathrm{ell}$ & $\%$ & 1000 th & $1 \%$ & $1000 \mathscr{A l}$ & $0 ;$ \\
\hline Versicherung auf den Todesfall.......... & $340284 \mathrm{I}$ & 84,2 & 1979097 & 84,7 & I 423744 & 83,7 \\
\hline$n \quad n \quad n$ Lebensfall ......... & 384472 & 9,5 & 199700 & 8,5 & 184772 & 10,8 \\
\hline Rentenversicherung $\ldots \ldots \ldots \ldots \ldots \ldots \ldots \ldots$ & 244094 & 6,1 & Ij I 861 & 6,5 & 92233 & 5,4 \\
\hline Sonstige Versicherungen $\ldots \ldots \ldots \ldots \ldots \ldots$ & 8497 & 0.2 & 75.37 & 0.31 & 960 & 0.1 \\
\hline Zusammen .... & 4039904 & $100,0^{1}$ & 2338195 & 100,0 & 1701709 & 100.0 \\
\hline
\end{tabular}

Die Verteilung des Gewinns ist in Tabelle I 41 dargestellt. Wenn dabei die Summe des Gesamtgewinns der Gegenseitigkeitsvereine nicht mit der Summe der Jahresüberschüsse und der Vorträge aus dem Vorjahre, wie sie in der Bilanz ('Tabelle I 38) ent- 
halten sind, übereinstimmt, so erklärt sich das daraus, daß auch die Verteilung des vom Allgemeinen Deutschen-Versicherungsvercin in Stuttgart aus dem Lebensversicherungsgeschäft erzielten Gewinns nachgewiesen ist, während die Bilanz dieser Gesellschaft bei Gruppe II gegeben ist.

Sonach standen für die Gewinnverteilung zur Verfügung bei den Aktiengesellschaften $85290528 \mathscr{M}$ und bei den Gegenseitigkeitsvereinen $59635024 \mathscr{N}$; diese Beträge sind folgendermaßen verteilt worden:

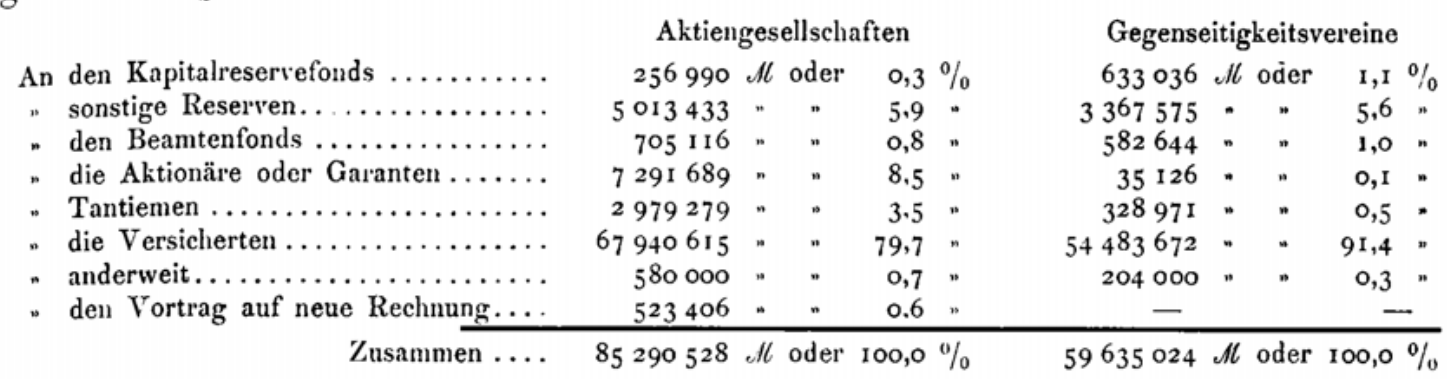

Hinsichtlich der den Versicherten zugewiesenen Beträge ist zu beachten, daß einige Gesellschaften den den Versicherten zustehenclen Gewinn in der Bilanz bereits verrechnet haben, so daß er hier nicht in die Erscheinung tritt. Diese Beträge (vgl. Fußnoten zu Tabelle I 41 und IV 21) haben im Berichtsjahre insgesamt 3,188 Millionen Mark ausgemacht.

Die Bilanzen der Versicherungseinrichtungen von Berufsvereinigungen mit den zugehörigen Erläuterungen sind in den Tabellen I 43 bis I 46 gegeben. Die Gesamtbilanz schließt ab mit $86911364 \mathscr{M}$; dic Hauptposten weisen folgende Beträge auf:

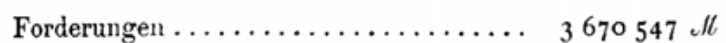

Kapitalanlagen ................ 82756304 .

Kassenbestand ................. 39609 "

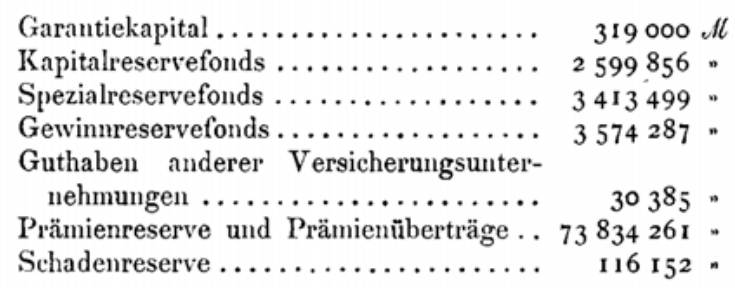

Schadenreserve............... $116 \mathrm{I}_{52}$.

Von den insgesamt $82756304 \mathscr{N}$ betragenden Kapitalanlagen kamen $795375 \mathscr{N}$ oder 1,0 Prozent auf Grundbesitz, $68463532 \mathscr{N}$ oder 82,7 Prozent auf Hypotheken, $3136725 \mathscr{N}$ oder 3 , s Prozent auf Darlehen an öffentliche Körperschaften, $8987456 \mathscr{N}$ oder 10,9 Prozent auf Wertpapiere und der Rest von $1373216 \mathscr{M}$ oder 1,6 Prozent auf Policedarlehen. Die Vergleichung des Bestandes am Ende des Vorjahrs mit dem Bestand am Ende des Berichtsjahrs ergibt eine Zunahme des Grundbesitzes um $14716 \mathscr{N}$, des Hypothekenbesitzes um $8085028 \mathscr{N}$, der Darlehen an öffentliche Körperschaften um $1021429 \mathscr{N}$, des Buchwertes der Wertpapiere um $720458 \mathscr{N}$ und der Policedarlehen um $166570 \mathscr{N}$.

Die Bilanzen von 17 Anstalten von Berufsvereinigungen weisen einen Gewinn von zusammen $2476946 \mathscr{M}$ auf, der wie folgt verteilt worden ist: $449449 \mathscr{N}$ oder 18,1 Prozent an den Kapitalreservefonds, $177238 \mathscr{M}$ oder 7,2 Prozent an die Spezialreserven, $13544 \mathscr{N}$ oder 0,5 Prozent an den Beamtenfonds, $20945 \mathscr{M}$ oder 0,s Prozent Tantiemen, $1757061 \mathscr{N}$ oder 70,9 Prozent an die Versicherten und $49513 \mathscr{N}$ oder 2,o Prozent Vortrag auf neue Rechnung.

Die Bilànzen von 20 ausländischen Gesellschaften, deren Lebensversicherungsge- Die aus. schäfte in dieser Statistik behandelt sind, finden sich in Tabelle I 47; von der Northern $\begin{gathered}\text { ländischen } \\ \text { Unterneh. } \\ \text { mungen }\end{gathered}$

Versicherungseinrichtungen von Berufsvereinigungen

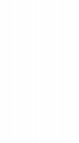


Assurance Company ist die Bilanz bei der Feuerversicherung in Tabelle IV 23 mitgeteilt. Die Bilanzen sind nach Möglichkeit den für die Bilanzen der deutschen Unternehmungen geltenden Grundsätzen angepaßt worden.

Von besonderem Interesse ist die Art der Kapitalanlagen (Tabelle I 48), die von den Gesellschaften der verschiedenen Länder, sei es aus eigenem Antriebe, sei es auf Veranlassung der Aufsichtsbehörden, bevorzugt wird; deshalb sind in der folgenden Übersicht die in der Tabelle I 48 aufgeführten ausländischen Gesellschaften nach Heimatländern zusammengefaßt und zum Vergleiche die Verhältniszahlen für die deutschen Unternehmungen wiederholt:

Von je $1000 \mathscr{N}$ Kapitalanlagen entfielen auf

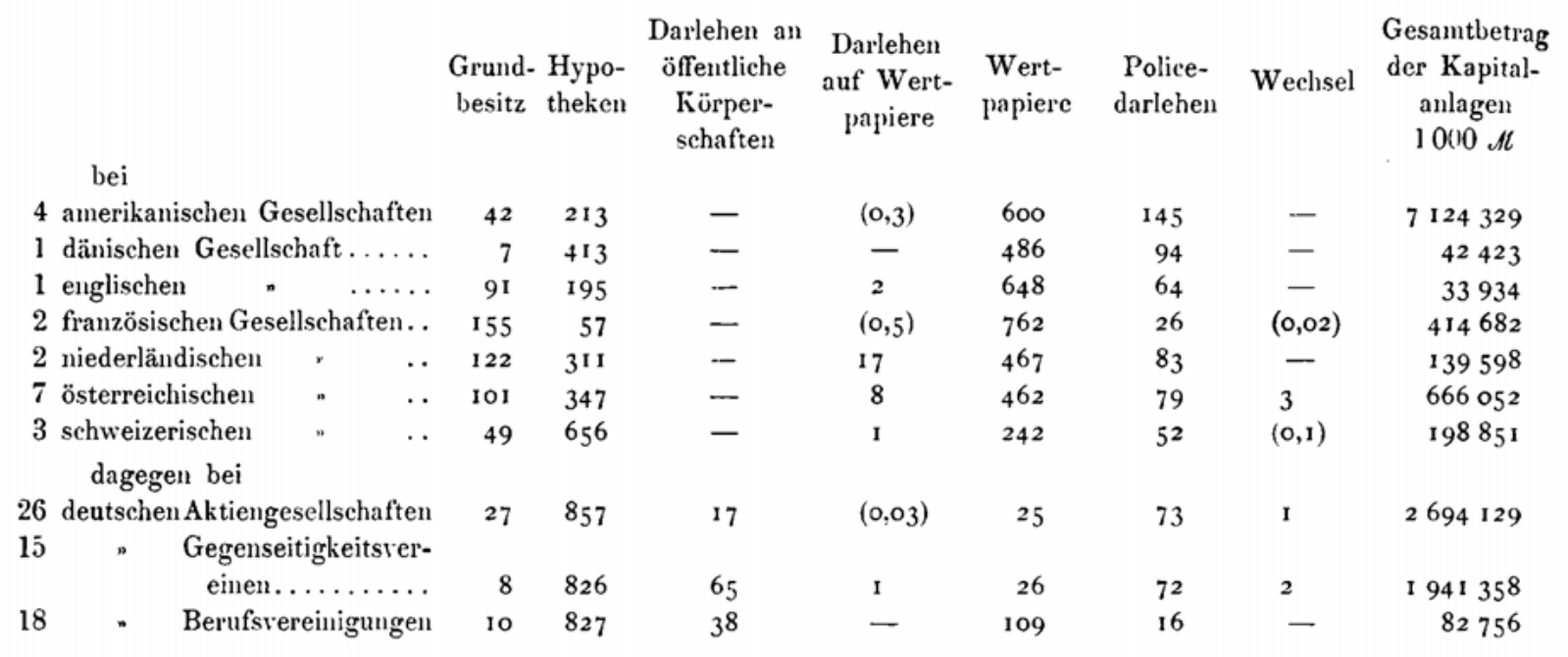

I)as auslăndische Gieschäft der deutschen Unternelımungen

Von den allgemeinen deutschen Unternehmungen haben die Kapitalversicherung im Auslande mittelbar oder unmittelbar betrieben: 21 in der Schweiz, 20 in Dänemark, je 19 in den Niederlanden und in Österreich, 17 in Belgien, 8 in Frankreich, je 7 in Italien, Norwegen, Rußland und Schweden, 6 in der Türkei, je 5 in Ägypten, Spanien, Ungarn und in den Vereinigten Staaten von Amerika, je 4 in England, Finnland und Rumänien, 3 in Luxemburg, je 2 in Argentinien, Bolivien, Brasilien, China und Portugal, je 1 in Griechenland, Japan, Liechtenstein und Montenegro. In der Rentenversicherung ist das Geschäft natürlich auch im Auslande weniger verbreitet; es haben gearbeitet: 12 Unternehmungen in der Schweiz, 11 in Belgien, 10 in den Niederlanden, 7 in Österreich, je 6 in Dänemark, Frankreich und Rußland, je 4 in Italien, Schweden und in der Türkei, je 3 in China, England, Finnland, Luxemburg, Norwegen und in den Vereinigten Staaten von Amerika, je 2 in Ägypten, Argentinien, Brasilien, Japan, Rumänien, Spanien und Ungarn, je 1 in Chile, Griechenland, Portugal und Zentralamerika.

In der Kapitalversicherung hatten diese Unternehmungen Ende 1910 im Auslande 247689 laufende Verträge über 1147,604 Millionen Mark, das sind 2,1 Prozent aller Versicherungen und 8,4 Prozent der gesamten Versicherungssummen; die vereinnahmten Prämien, einschließlich der für Rückversicherungen wieder abgegebenen Prämien, betrugen 49,879 Millionen Mark, wovon 47,907 Millionen oder 96,0 Prozent für selbst algeschlossene und 1,9i2 Millionen oder 4,0 Prozent für in Rückdeckung übernommene Versicherungen zu rechnen sind. An Versicherungsleistungen wurden 20,122 Millionen 
Mark gewährt, nämlich 19,036 Millionen für selbst abgeschlossene Versicherungen und 1,08c Millionen für Rückversicherungen; von den in der ausländischen Kapitalversicherung überhaupt fällig gewordenen Beträgen kommen demnach 5,4 Prozent auf die in Rückdeckung übernommenen Versicherungen.

In der Rentenversicherung liefen Ende 1910 im Auslande 3583 Policen über 1,:582 Millionen Mark Jahresrente, das sind 4,6 Prozent aller Policen und 5,1 Prozent der gesamten Jahresrente. An Prämien wurden vereinnahmt 1,300 Millionen Mark. Für Versicherungsleistungen waren 1,060 Millionen Mark bereitzustellen.

Zu einer eingehenden Vergleichung des ausländischen Geschäfts aller deutschen Lebensversicherungs-Unternehmungen mit dern deutschen Geschäfte der ausländischen Gesellschaften fehlt das Material, doch bieten folgende Zahlen wenigstens einige Anhaltspunkte:

Im Jahre 1910 hat die Prämieneinnahme betragen:

\begin{tabular}{|c|c|c|c|}
\hline & $\begin{array}{l}\text { insgesamt } \\
1000 \mathscr{A t}\end{array}$ & $\begin{array}{l}\text { für selbst ab- } \\
\text { geschlossene } \\
\text { Versicherungen } \\
1000 \mathscr{M}\end{array}$ & $\begin{array}{c}\text { für in Rückdeckung } \\
\text { übernommene } \\
\text { Versicherungen } \\
1000 \mathscr{M}\end{array}$ \\
\hline $\begin{array}{l}\text { bei den deutschen Gesellschaften aus dem aus- } \\
\text { ländischen Geschäfte..................... } \\
\text { bei den ausländischen Gesellschaften aus dem }\end{array}$ & 51179 & 49207 & I 972 \\
\hline deutschen Geschäfte $\ldots \ldots \ldots \ldots \ldots \ldots \ldots$ & 43987 & 43 I 74 & $8{ }_{3}$ \\
\hline
\end{tabular}

Hiernach haben insgesamt die deutschen Gesellschaften für Lebensversicherungen 7,192 Millionen Mark mehr an Prämien aus dem Auslande bezogen als die ausländischen Gesellschaften aus dem Deutschen Reiche eingenommen haben. Beträchtlich größer sind im Berichtsjahre die Unterschiede hinsichtlich der Zahlungen an Versicherungsleistungen; diese haben betragen:

\begin{tabular}{|c|c|c|c|}
\hline & insgesamt & $\begin{array}{l}\text { für selbst ab- } \\
\text { geschlossene } \\
\text { Versicherungen }\end{array}$ & $\begin{array}{c}\text { für in Rũckdeckung } \\
\text { übernommene } \\
\text { Versicherungen }\end{array}$ \\
\hline bei den deutschen Gesellschıften für ausländische & $1000 . / 1$ & $1000 \mathscr{N}$ & $1000 \mathscr{M}$ \\
\hline $\begin{array}{l}\text { Versicherungen } \ldots \ldots \ldots \ldots \ldots \ldots \ldots \ldots \ldots \ldots \ldots \ldots \\
\text { bei den ausländischen Gesellschaften für deutsche }\end{array}$ & 21182 & 20096 & 1086 \\
\hline Versicherungen $\ldots \ldots \ldots \ldots \ldots \ldots \ldots \ldots \ldots$ & 36949 & 36639 & 310 \\
\hline
\end{tabular}

Außer den besprochenen und in den Tabellen behandelten deutschen Versiche- Die kleinen rungsunternehmungen steht, wie bereits erwähnt, gemäß $\$ 2$ V.A.G. noch eine Anzahl kleiner Sterbe- und Pensionskassen unter Reichsaufsicht, die für eng begrenzte Kreise Pensionsund Stẹrbebestimmt und vielfach von gewerblichen Firmen nur für die eigenen Angestellten oder Arbeiter eingerichtet sind. Die Zahl dieser Gegenseitigkeitsvereine ist schwankend; einerseits sind diese Unternchmungen wohl kaum sämtlich bekannt, auch entstehen stets neue derartige Vereine; anderseits geht die Beaufsichtigung nicht selten gemäß $\S 3$ Abs. 2 nachträglich an die Landesbehörde über. Andere Vereine verfallen der Auflösung. Wie dem auch sei, die Zahl der unter Reichsaufsicht stehenden Sterbekassen und Pensionskassen wird immer nur sehr klein sein im Vergleiche zu der Zahl der unter Landesaufsicht befindlichen Kassen; wenn hier einige Mitteilungen über die unter unserer Aufsicht stehenden Kassen gemacht werden, so können daraus keineswegs Schlüsse über die Bedeutung des Sterbekassen- und Pensionskassenwesens im Reiche gezogen werden. 
Für das Jahr I910 haben insgesamt 113 solcher Sterbekassen dem Aufsichts. amte befriedigende Jahresberichte eingereicht. Danach ist die Zahl der Mitglieder Ende 1910 auf rund 510360 anzusetzen; die Einnahmen haben im Berichtsjahre $7249857 \mathscr{M}$ betragen, die Ausgaben $3656270 \mathscr{M}$.

Von den Einnahmen kommen $5597447 \mu$ oder 77,2 Prozent auf Beiträge für die Mitglieder, 55 732 $\|$ oder 0,8 Prozent auf Eintrittsgelder, $1232727 \mathscr{M}$ oder 17,0 Prozent auf Vermögenserträge und $363951 \mathscr{M}$ oder 5,0 Prozent auf andere Einnahmen. Von den Ausgaben kommen $2090873 \mathscr{N}$ oder 57,2 Prozent auf Sterbegelder, 326 $196 \mathscr{M}$ oder 8,9 Prozent auf Krankengelder, $120025 \mathscr{M}$ oder 3,3 Prozent auf Zahlungen für vorzeitig aufgelöste Versicherungen, 205115 d/ oder 5,6 Prozent auf Gewinnanteile, $44862 !) \mathscr{N}$ oder 12,3 Prozent auf Verwaltungskosten und Steuern und $465432 \mathscr{N}$ oder 12,i Prozent auf die übrigen Ausgaben. Das Vermögen dieser Kassen belief sich am Schlusse des Jahres i 9 го auf $32569989 \mathscr{M}$.

Die Zahl der Pensionskassen der genannten Art, die in befriedigender Weise über ihren Geschäftsbetrieb im Jahre I910 an das Amt berichtet haben, betrug 86; diese Kassen hatten Ende r 909 93073 aktive Mitglieder und an Bezugsberechtigten 4493 Pensionierte, 2504 Witwen und 1030 Waisen und Ende r 9 10 $100093 \mathrm{\Lambda ktive}$, 4617 Pensionierte, 2672 Witwen und 1136 Waisen. Die Einnahmen betrugen im Berichtsjahre $18698484 \mathscr{M}$, während $5345517 \mathscr{M}$ verausgabt wurden. Von den Einnahmen entfallen $12600334 \mathscr{N}$ oder 67,4 Prozent auf Beiträge für die Mitglieder, 2う5 935 $\mathscr{N}$ oder 1,4 Prozent auf Eintrittsgelder, $4234453 \mathscr{N}$ oder 22,6 Prozent auf Vermögenserträge und $1607762 \mathscr{A}$ oder 8,6 Prozent auf anderweitige Einnahmen. Von den Ausgaben entfallen $3901249 \mathscr{N}$ oder 73,0 Prozent auf Pensionen, $85897 \mathscr{M}$ oder 1,6 Prozent auf Krankengeld, $86993 \mathscr{M}$ oder 1,6 Prozent auf Sterbegeld und sonstige Leistungen an Versicherte, $466286 \mu$ oder 8,7 Prozent auf Abfindungen beim Austritt aus der Kasse (meist infolge Wechsels der Dienststelle), $498328 \mathscr{M}$ oder 9,3 Prozent auf Verwaltungskosten und Steuern und $306764 \mathscr{M}$ oder 5,8 Prozent auf die übrigen Ausgaben. Der niedrige Satz für Verwaltungskosten hat seinen Grund in erster Linie darin, daß bei den von gewerblichen Firmen eingerichteten Versicherungskassen die Verwaltungskosten meistens vollständig von den Firmen übernommen werden und somit die Kassen nicht belasten. Das Vermögen dieser Pensionskassen belief sich am Ende des Berichtsjahrs auf $112730205 \mathscr{M}$.

\section{Gruppe II, Unfall- und Haftpflichtversicherung}

Von den großen deutschen Versicherungsunternehmungen, welche der Reichsaufsicht unterstehen, waren, wie im Vorjahr, auch im Jahre r9ı 28 auf dem Gebiete der Unfall- und Haftpflichtversicherung tätig, 27 Aktiengesellschaften und 1 Versicherungsverein auf Gegenseitigkeit. Der Nordstern, Unfall- und Alters-Versicherungs-AktienGesellschaft zu Berlin zeichnet jetzt Nordstern, Unfall- und Haftpflicht-VersicherungsAktien-Gesellschaft zu Berlin.

Außerdem berichtet die vorliegende Statistik wieder über acht ausländische Gesellschaften.

Ausgeschieden ist die $\mathrm{k} . \mathrm{k}$. privilegierte Versicherungs-Gesellschaft Österreichischer Phönix in Wien und neu hinzugekommen die Providentia, Allgemeine Versicherungs- 
gesellschaft in Wien, die auch das deutsche Unfall- und Haftpflichtversicherungsgeschäft jener Gesellschaft vom Beginne des Berichtsjahrs ab übernommen hat.

Zur Beurteilung des Umfanges des Geschäfts der einzelnen Gesellschaften soll die Tabelle II 1 dienen. Daraus ist zu ersehen, daß bei den deutschen Unternehmungen am Ende des Jahres 1910 ohne die vom Allgemeinen Deutschen Versicherungs-Verein in Stuttgart in Rückdeckung genommenen Versicherungen in der Unfallversicherung 1158892 und in der Haftpflichtversicherung 2262130 Policen über selbst abgeschlossene oder in Rückdeckung übernommene Versicherungen in Kraft waren.

In der Haftpflichtrersicherung wurden Versicherungen mit Gewinnbeteiligung außer ron dem Allgemeinen Deutschen Versicherungs-Verein in Stuttgart auf Gegenseitigkeit nur von der Oberrheinischen Versicherungs-Gesellschaft in Mannheim, und zwar nur in ganz geringem Umfang abgeschlossen. Auch in der Unfallversicherung kommen Versicherungen mit Gewinnbeteiligung nur bei wenigen Unternehmungen in Betracht. Von den Aktiengesellschaften haben nur vier solche Versicherungen abgeschlossen, darunter zwei auch nur in geringem Umfange. Von der Prämieneinnahme des Geschäftsjahrs in Höhe von $42336665 \mathscr{N}$ für selbst abgeschlossene Unfallversicherungen sämtlicher Unternehmungen kamen auf Versicherungen mit Gewinnbeteiligung nur $11841148 \mathscr{M}$ oder 28,0 Prozent, oder wenn man den Allgemeinen Deutschen Versicherungs-Verein in Stuttgart, weil er eine Gewinnbeteiligung nur auf Grund seines Charakters āls Gegenseitigkeitsverein kennt, ausscheidet, 16,i Prozent.

Für das Rückversicherungsgeschäft lassen sich folgende Zahlen angeben. In der Unfallversicherung waren in Rückdeckung übernommen für $2483573 \mathscr{M}$ Prämie oder 5,5 vom Hundert der ganzen Prämieneinnalıme von $44820238 \mathscr{M}$ und in Rückdeckung gegeben für $7613022 \mathscr{M}$ oder für 17,0 vom Hundert der ganzen Prämieneinnahme. In der Haftpflichtversicherung waren in Rückdeckung übernommen für $1859268 \mathscr{M}$ Prämie oder 3,9 vom Hundert und in Rückdeckung gegeben für $12228313 \mathscr{M}$ oder 25,8 vom Hundert der gesamten Prämieneinnahme von $47356360 \mathscr{M}$.

In dicsen wie in allen folgenden Zahlen sind bei dem Allgemeinen Deutschen Versicherungs-Verein in Stuttgart in der Haftpflichtversicherung auch die auf die Versicherung gegen Wasserleitungsschäden und einige andere kleine Sachversicherungszweige bezüglichen Zahlen enthalten.

Vergleicht man den Versicherungsbestand am Ende des Berichtsjahrs mit dem entsprechenden Bestand am Ende des Vorjahrs (Tabelle II 2), so findet man, daß die Zahl der Policen bei der Unfallversicherung um 91454 oder 8,6 Prozent und bei der Haftpflichtversicherung um 140137 oder 6,6 Prozent des Bestandes am Ende des Vorjahrs zugenommen hat. Die gesamte Prämieneinnahme hat im Geschäftsjahre bei der Unfallversicherung $2949630 \mathscr{N}$ oder 7,0 Prozent und bei der Haftpflichtversicherung $3716643 \mathscr{M}$ oder 8,5 Prozent mehr als im Vorjahre betragen.

In unseren Statistiken sind von vornherein die den einzelnen Versicherungszweigen eigentümlichen und in den Büchern getrennt behandelten Posten - anfänglich unter der Bezeichnung Sonder-Einnahmen und Sonder-Ausgaben, seit dem Jahre I 906 unter der Bezeichnung Betriebs-Einnahmen und Betriebs-Ausgaben — einzeln aufgeführt worden; seit 1905 konnten auch die allgemeinen Verwaltungskosten und die Steuern mit befriedigender Genauigkeit nach Geschäftszweigen getrennt werden. Seitdem ist

Vergleich mit dem Vorjahre

$$
\begin{aligned}
& \text { Die } \\
& \text { Betriebs- } \\
& \text { Einnahmen } \\
& \text { und } \\
& \text { Ausgaben } \\
& \text { des } \\
& \text { Berichts- } \\
& \text { jahrs }
\end{aligned}
$$


der Gewinn oder Verlust aus dem eigentlichen Versicherungsbetriebe des Geschäfts. jahrs bei jedem Zweige ermittelt worden; Einnahmen und Ausgaben, die nicht unmittel. bar mit dem Versicherungsbetriebe des Berichtsjahrs zusammenhängen, wie z. B. bei der Schadenversicherung die Erträge der Vermögensverwaltung, bleiben dabei außer Betracht (vgl. "Entwickelung “ S. 21). Bei der Unfall- und Haftpflichtversicherung ist indessen der Betriebsgewinn oder Verlust nicht vollständig ermittelt worden, weil bei den Betriebs. Einnahmen der Ertrag der die Prämienreserve deckenden Kapitalanlagen nicht sicher anzugeben ist. Schätzungen des Amts sollen aber in dieser Statistik vermieden werden, deshalb ist, um zugleich alle Mißverständnisse auszuschließen, in Tabelle II 3 kein Gewinn oder Verlust eingestellt. Das Erträgnis der Vermögensverwaltung der Gesellschaften im ganzen, aber unter möglichster Beschränkung auf die der Unfäll- und Haftpflichtversicherung zugerechneten Vermögensteile, findet sich in Tabelle II 5. Der Gewinn oder Verlust des alle Zweige umfassenden Gesamtgeschäfts wird in der Bilanz ausgewiesen.

Als Betriebs-Einnahmen sind bei Gruppe II verrechnet die Prämien für das Geschäftsjahr, d.h. die Prämien unter Berücksichtigung der Ưberträge aus dem Vorjahr und der Überträge auf das folgende Jahr, einschließlich der an die Rürkversicherungsgesellschaften gezahlten Rückversicherungsprämien, ferner dic Policegebühren, die Vergütungen der Rückversicherer, der Unterschied zwischen der Schadenreserve aus dem Vorjahr und den Zahlungen und Rückstellungen für Versicherungsfälle der Vorjahre aus selbst abgeschlossenen Versicherungen, sofern die Schadenreserve größer ist, und der etwaige Mehrbetrag der aus dem Vorjahr übernommenen Prämienreserve gegenüber der am Schlusse des Jahres zu stellenden Prämienreserve. Die Betriebs-Ausgaben setzen sich bei Gruppe II zusammen aus den Zahlungen für die selbst abgeschlossenen und für die in Rückdeckung übernommenen Versicherungen einschließlich des Anteils der Rückversicherer, aus den Rückversicherungsprämien, dem Unterschiede zwischen der Schadenreserve aus dem Vorjahr und den Zahlungen und Rückstellungen für Versicherungsfälle der Vorjahre aus selbst abgeschlossenen Versicherungen, sofern die Schadenreserve kleiner ist, und endlich aus der Zunahme der Prämienreserve. Es soll hier aber besonders betont werden, daß der Unterschied zwischen der Schadenreserve aus dem Vorjahr und den Zahlungen und Rückstellungen für Versicherungsfälle aus selbst abgeschlossenen Versicherungen der Vorjahre allein keinen Schluß darüber zuläßt, ob die Schadenreserve zulänglich bemessen war. Denn einerseits sind aus der Schadenreserve auch andere Leistungen als Zahlungen für Versicherungsfälle der Vorjahre zu bewirken, z. B. Zahlungen für die in Rückdeckung übernommenen Versicherungen und Zuführungen zur Prämienreserve. Anderseits wird die Schadenreserve meist ohne den Anteil der Rückversicherer eingestellt, wogegen die Zahlungen einschließlich dieses Anteils erscheinen. Auch enthalten die Zahlungen für Versicherungsfälle der Vorjahre Beträge, die nicht aus der Schadenreserve, sondern aus der Prämienreserve zu decken sind.

Die Betriebs-Einnahmen des Berichtsjahrs haben ohne die Erträge der Prämienreserven insgesamt bei den 28 deutschen Gesellschaften $110757398 \mathscr{M}$ betragen; davon kamen auf Prämien für Unfallversicherung $44403573 \mathscr{M}$ oder 40,1 vom Hundert, auf Prämien für Haftpflichtversicherung $46180659 \mathscr{M}$ oder 41,7 vom Hundert und auf Vergütungen der Rückversicherer 15980277 $\mathscr{N}$ oder 14,4 vom Hundert aller dieser Betriebs-Einnahmen.

Die Summe der Betricbs-Ausgaben olıne Verwaltungskosten beziffert sich auf $70862001 \mathscr{H}$; davon entfallen 40534553 $\mathscr{M}$ auf Zahlungen 1ür Versicherungsfälle 
im Geschäftsjahr aus selbst abgeschlossenen Versicherungen, und zwar (Tabelle II 4) $19512140 \mathscr{M}$ oder $2 \bar{\imath}, 5$ Prozent aller Betriebs-Ausgaben auf Unfallversicherungsfälle, ferner $17404487 \mathscr{M}$ oder 24,6 Prozent auf Haftpflichtversicherungsfälle, $1652084 \mathscr{M}$ oder 2,3 Prozent auf laufende Renten und endlich $1966142 \mathscr{M}$ oder 2,s Prozent auf Prämienrückgewährbeträge. Von der Summe der Betriebs-Ausgaben entfallen ferner $4668589 \mathscr{M}$ oder 6,c, Prozent auf Vergütungen für die in Rückdeckung übernommenen Versicherungen (darunter $1990992 \mathscr{M}$ oder 2,8 Prozent für eingetretene Unfallversicherungsfälle und $1225232 \mathscr{N}$ oder 1,; Prozent für eingetretene Haftpflichtversicherungsfälle), $7613022 \mathscr{A}$ oder 10,7 Prozent auf Rückversicherungsprämien für Unfallversicherungen und $12228313 \mathscr{N}$ oder 17,2 Prozent auf Rückversicherungsprämien für Haftpflichtversicherungen.

Die 28 Gesellschaften haben an Verwaltungskosten und Steuern insgesamt $33151463, \mathscr{A}$ für das Unfall- und Haftpflichtversicherungsgeschäft aufgewandt.

Wenn man annimmt, daß die Vergütungen für die in Rückdeckung übernommenen Versicherungen sich nur auf Versicherungsfälle des Geschäftsjahrs beziehen, und davon absieht, daß ein Teil der laufenden Renten, die ja in ihrer Gesamtheit nur geringe Beträge erfordern, aus früheren Jahren herrührt, so kann man den Gesamtbetrag der Schadenzahlungen (45555102 $\mathscr{N}$ ) in Beziehung setzen zur Prämieneinnahme für das Gesclı̈̈ftsjahr (90584232 $\mathscr{H}$ ). Man findet dann, daß von je $1000 \mathscr{M}$ Prämieneinnalıme $503 \mathscr{M}$ für Schadenzahlungen verausgabt wurden; weitere $46 \mathscr{M}$ wurden zur Erhöhung der Prämienreserven verwandt. Tatsächlich ist allerdings für eigentliche Schäden etwas weniger aufgewandt worden; denn einzelne Gesellschaften verrechnen bei den Schadenzahlen auch noch mancherlei andere Beträge, z. B. Regulierungskosten. - In den letzten fünf Jahren haben diese Zahlen betragen:

$\begin{array}{lrrrrr} & 1906 & 1907 & 1908 & 1909 & 1910 \\ \text { bei den Schadenzahlungen.................... } & 493 & 498 & 481 & 497 & 503 \\ \text { bei den Zuführungen zur Prämienreserve.... } & 65 & 66 & 59 & 49 & 46\end{array}$

Das Ergebnis der Vermögensverwaltung wird von den Unternehmungen in der Regel nicht nach den einzelnen Geschäftszweigen getrennt; nur der Lebensversicherung werden immer bestimmte Erträge überwiesen, weil das der Betrieb dieses Geschäfts erfordert. Es besteht keine Veranlassung, in dieser Statistik von dem Verfahren der Gesellschaften abzugehen. Soweit also nicht ein Teil des Ertrags einem bestimmtem Zweige zugewiesen ist, wird das Ergebnis nur insgesamt aufgeführt und im allgemeinen bei derjenigen Gruppe mitgeteilt, zu der das Hauptgeschäft der Unternehmung gehört.

In Tabelle II 5 findet sich bei 6 Gesellschaften der Ertrag der gesamten Vermögensverwaltung, bei 11 Gesellschaften nur der nicht der Lebensversicherung und bei 2 Gesellschaften der nicht der Feuerrückversicherung zukommende Teil; für die übrigen 9 Unternehmungen ist der Ertrag entweder in Tabelle I 29 oder in Tabelle IV 16 nachgewiesen.

Unter den Erträgen stehen wie überall so auch hier die Zinsen im Vordergrund, nämlich mit $8225959 \mathscr{N}$; der Kursgewinn bleibt um $64715 \mathscr{M}$ hinter dem Kursverluste zurück, der sonstige Gewinn übertrifft den sonstigen Verlust um $199333 \mathscr{M}$. Als reiner Ertrag sind $8676459 \mathscr{M}$ zu verzeichnen.

Eine Bilanz ist in dieser Statistik grundsätzlich nur für das Gesamtgeschäft einer Die Bilanx jeden Gesellschaft gegeben und bei derjenigen Gruppe aufgeführt, zu der das Unter- 
nehmen seinem Hauptbetriebe nach - Transportversicherung immer ausgeschlossen gehört. So bleiben bei Gruppe II von den 28 deutschen Gesellschaften, welche Unfalloder Haftpflichtversicherungen direkt abschließen, nur 9 Anstalten übrig. Im übrigen finden sich die Bilanzen von Deutschland, Victoria, Urania, Providentia, Janus, Teutonia, Atlas, Magdeburger Lebens-Versicherungs-Gesellschaft, Wilhelma, Bayerische Versicherungs-Bank, Nürnberger Lebens-Versicherungs-Bank und Germania bei Gruppe I (Tabelle I 38) und die Bilanzen von Thuringia, Neptun, Albingia, Nord-Deutsche Versicherungs-Gesellschaft, Rheinland, Prcußische National-Versicherungs-Gesellschaft und Stuttgarter Mit- und Rückversicherungs-Aktien-Gesellschaft bei Gruppe IV (Tabelle IV 19).

Aus den in Tabelle II 6 für die 9 Gesellschaften gegebenen Bilanzen mögen hier die folgenden Schlußzahlen Platz finden:

Verpflichtungen der Aktionäre....... $36478800 \AA$

Kapitalanlagen ................ 177186754 *

Forderungen .................. 33200104 "

Kassenbestand $\ldots \ldots \ldots \ldots \ldots \ldots \ldots \ldots \ldots \ldots \ldots, \quad 383873 \cdot$

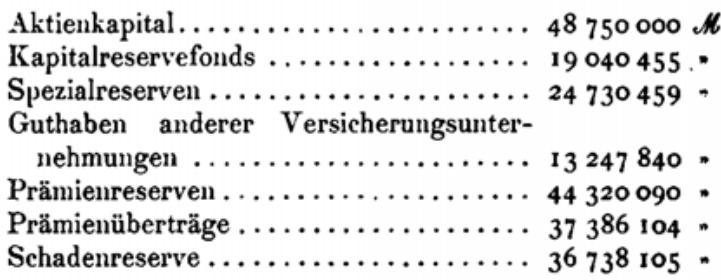

Dabei ist zu erwähnen, daß aus den Nebenbetrieben der Gesellschaften von den Prämienreserven $32334453 \mathscr{M}$ auf Lebensversicherungen und von den Prämienüberträgen $18023776 \mathscr{M}$ auf nicht zur Gruppe II gehörige Versicherungszweige entfallen; für die Unfall- und Haftpflichtversicherung bleiben danach an Prämienreserven nur $11985637 \mathscr{M}$ und an Uberträgen $19362328 \mathscr{M}$.

Die Kapitalanlagen (177 186 754 $\mathcal{N})$ verteilen sich auf folgende Wertarten: $14268856 \mathscr{M}$ oder 8,1 Prozent Grundbesitz, $110735175 \mathscr{M}$ oder 62,5 Prozent Hypotheken, $12766940 \mathscr{M}$ oder 7,2 Prozent Darlehen an öffentliche Körperschaften, $35327572 \mathscr{N}$ oder 19,9 Prozent Wertpapiere, $1549043 \mathscr{M}$ oder 0,9 Prozent Policedarlehen und $2539168 \mathscr{M}$ oder 1,4 Prozent Wechsel. Stellt man diese Zahlen denen des Vorjahrs gegenüber, so haben der Grundbesitz um $2574136 \mathscr{M}$, der Besitz an Hypotheken um $642256 \mathscr{M}$, die Wertpapiere um $1525381 \mathscr{M}$, die Policedarlehen um $175308 \mathscr{M}$, die Darlehen an öffentliche Körperschaften um $12766940 \mathscr{M}$ und der Bestand an Wechseln um $360002 \mathscr{M}$ zugenommen. Sieht man von den Policedarlehen, die den öffentlichen Geldverkehr nicht direkt berühren, ab, so haben jene 9 Gesellschaften dem Kapitalmarkte $17868715 \mathscr{N}$ zugeführt. Dem Nennwerte nach haben die Wertpapiere um $1760000 \mathscr{M}$ zugenommen.

Jede der 9 Gesellschaften hat mit einem Gewinn abgeschlossen, der sich für alle Gesellschaften zusammen nach der Bilanz auf $12469333 \mathscr{N}$ und mit Einschluß des Gewinnvortrags aus dem Vorjahr auf $14258399 \mathscr{M}$ stellt gegen $11616932 \mathscr{M}$ und $12988384 \mathscr{M}$ im Vorjahre. Von dem Gewinne des Berichtsjahrs entfallen auf die Lebensversicherungsabteilung des Allgemeinen Deutschen Versicherungs-Vereins in Stuttgart $1338540 \mathscr{M}$, dessen Verteilung aus Tabelle I 41 zu ersehen ist. Von dem Reste, $12919859 \mathscr{M}$, sind nach Tabelle II 8 überwiesen $38178 \mathscr{N}(0,3$ Prozent $)$ an den Kapitalreservefonds, $956745 \mathscr{H}(7,4$ Prozent) an sonstige Reserven, $298930 \mathscr{N}(2,3$ Prozent) an die Beamtenfonds, $4236848 \mathscr{N}$ (32,8 Prozent) an die Aktionäre; ferner sind ausgegeben $1262588 \mathscr{N}(9,8$ Prozent) an Tantiemen, $3231141 \mathscr{M}$ (25,0 Prozent) Gewinnanteile an die Versicherten sowie $681480 \mathscr{M}(5,3$ Prozent) anderweit; endlich sind $2213949 \mathscr{M}$ (17,1 Prozent) auf neue Rechnung vorgetragen. 
In der diesjährigen Bilanz stehen die Kapitalreserven um $462108 \mathscr{M}$ und die Spezialreserven um $2844390 \mathscr{M}$ höher als in der Bilanz für Ende 1909.

Von den hier behandelten 28 deutschen Unternehmungen haben 17 Anstalten auch mehr oder weniger umfangreiche Unfall- und Haftpflichtversicherungsgeschäfte im Auslande abgeschlossen, nämlich in Finnland, Japan und in den Vereinigten Staaten von Amerika je 1, in Griechenland, Italien, Portugal, Spanien und in der Türkei je 2, in Rumänien 3, in Fngland, Luxemburg und Schweden je 4, in Rußland, Ungarn und Norwegen je 5, in Frankreich 9, in Belgien und Dänemark je 10, in Österreich 12, in der Schweiz 13 und endlich in den Niederlanden 15.

Diese Unternehmungen hatten im Auslande 137174 Versicherungen in Kraft, das sind 4,0 Pro\%ent aller Versicherungen. Die vereinnahmte Prämie, einschließlich der für Rückversicherungen wieder abgegebenen Teile, betrug für das ausländische Geschäft insgesamt 9,502 Millionen Mark, wovon 7,527 Millionen Mark für die selbst abgeschlossenen und 1,975 Millionen Mark für die in Rückdeckung übernommenen Versicherungen zu rechnen sind; im Verhältnisse zum Gesamtgeschäfte sind das 10,3 Prozent, 8,6 Prozent und 45,5 Prozent. Beim ausländischen Geschäfte machten die Prämien für das in Rückdeckung übernommene Geschäft 20,8 Prozent von den Prämien für das ganze ausländische Geschäft aus. Für Versicherungsleistungen wurden 5,634 Millionen Mark gewährt, nämlich 4,334 Millionen Mark für selbst abgeschlossene Versicherungen und 1,300 Nillionen Mark für Rückversicherungen; von der Gesamtausgabe für ausländische Schäden kommen demnach 22,9 Prozent auf in Rückdeckung übernommene Versicherungen. Auf $1000 \mathscr{M}$ Prämieneinnahme kommen $593 \mathscr{M}$ Schadenzahlungen.

Außer den erwähnten großen Unternehmungen unterlagen der Reichsaufsicht noch einige Gegenseitigkeitsvereine und einige Eingetragene Genossenschaften m. b. H. von geringerer Bedeutung, die ihre Mitglieder gegen Haftpflicht oder auch gegen Unfall und Haftpflicht versichern. Eine Änderung gegenüber dem Vorjahre hat nicht stattgefunden, so daß hier wieder die Hauptergebnisse von 10 solchen Vereinen mitgeteilt werden können.

Die Zahl der Policen dieser 10 Unternehmungen belief sich Ende I9 IO auf 52 714. Die Prämien für das Geschäfsjahr betrugen für alle Unternehmungen zusammen $590333 \mathscr{M}$. Davon wurden für Rückversicherungen $129188 \mathscr{M}$ wieder abgeführt. Verausgabt wurden für Schäden $279800 \mu$ und für Verwaltungskosten $107502 \mu$. Das Geschäftsjahr hat für alle Vereine einen Gewinn von insgesamt $225313 \mathscr{M}$ (einschließlich $5128 \mathscr{M}$ Gewinnvortrag aus dem Vorjahre) gebracht. Davon sind $195709 \mathscr{N}$ den Kapitalreservefonds, $8841 \mathscr{N}$ den sonstigen Reserven und $675 \mathscr{N}$ den Garanten überwiesen worden; ferner sind $6100 \mathscr{M}$ an Tantiemen ausgegeben, $5043 \mathscr{M}$ den Versicherten gutgebracht und $4250 \mathscr{M}$ auf neue Rechnung vorgetragen.

Die Bilanz dieser Unternehmungen schließt mit $2634136 \mathscr{M}$ ab. Als Hauptposten der Aktiva sind zu vermerken $1627354 \mathscr{M}$ Kapitalanlagen, $513179 \mathscr{N}$ Forderungen und $9211 \mathscr{M}$ Kassenbestand; als Hauptposten der Passiva kommen in Betracht $790555 \mathscr{M}$ Garantiekapital, $1019545 \mathscr{M}$ Kapitalreservefonds, $143564 \mathscr{M}$ Prämienüberträge und $368985 \mathscr{M}$ Schadenreserve. 

rungen abgeschlossen hat. Von den anderen Gesellschaften betrieben in Deutschland neben der Unfall- und Haftpflichtversicherung die Zürich auch die Einbruchdiebstahl. und die Kautionsversicherung, die Schweizerische National-Versicherungs-Gesellschaft auch die Einbruchdiebstahl-, die Glasversicherung und die Transportversicherung, die Schweizerische Unfallversicherungs-Aktiengesellschaft in Winterthur die Einbruchdiebstahlversicherung und die Kautions- und Garantieversicherung.

Acht ausländische Gesellschaften haben aus ihrem Unfallversicherungsgeschäft im Deutschen Reiche 19 10 $8136033 \mathscr{M}$ Prämien für selbst abgeschlossene Versicherungen und $12 \pi 01 \mathscr{M}$ Prämien für dic in Rückdeckung übernommenen Versicherungen eingenommen, wovon $1196012 \mathscr{N}$ für Rückversicherung wieder ausgegeben wurden.

An dem gesamten Geschäfte der Gesellschaften in der Unfallversicherung war das deutsche Geschäft mit 15,; Prozent der Prämieneinnahme und mit 25,0 Prozent der Ausgabe für Rückversicherungsprämien beteiligt.

In der Haftpflichtversicherung haben sechs ausländische Gesellschaften aus dem deutschen Geschäft $8687442 \mathcal{M}$ eingenommen, davon $178515 \mathscr{N}$ oder 2, 1 Prozent für die in Rückdeckung übernommenen Versicherungen; diese Prämieneinnahme betrug 42,1 Prozent des gesamten Haftpflichtversicherungsgeschäfts. Es wurden in dem deutschen Geschäfte $2463761 \mathscr{M}$ oder 28,4 Prozent der Prämien, in dem Gesamtgeschäft 11,9 Prozent der Prämien für Rückversicherungen wieder ausgegeben.

Für selbst abgeschlossene Versicherungen hatten die Gesellschaften folgende Beträge bereitzustellen : $4001241 \mathscr{N}$ für Unfallversicherungsfälle, $3105814 \mathscr{N}$ für Haftpflichtversicherungsfälle, $251989 \mathscr{N}$ für laufende Renten und $56771 \mathscr{M}$ für Prämienrückgewährbeträge, zusammen $7415815 \mathscr{M}$, für Versicherungsfälle aus den in Rückdeckung übernommenen Versicherungen $44227 \mathscr{N}$ für Prämienreserveergänzung, $24852 \mathscr{N}$ für eingetretene Unfallversicherungsfälle, $190661 \mathcal{N}$ für Haftpflichtversicherungsfälle und $28188 \mathscr{M}$ für sonstige Leistungen, zusammen $287928 \mathscr{M}$. Im Geschäftsjahr 1910 haben diese Gesellschaften insgesamt $7703743 \mathscr{M}$ für Schadenfälle, für Renten und für Prämienreserveergänzung verausgabt; in welchem Umfange hieran die Rückversicherer beteiligt sind, kann nicht angegeben werden. Diesen Schäden steht eine Bruttoprämieneinnahme - allerdings ohne Berücksichtigung der Überträge - von $16836176 \mathscr{M}$ gegenüber; von $1000 \mathscr{N}$ Prämien sind demnach durchschnittlich $458 \mathscr{N}$ für Schäden ausgegeben.

Im Vergleiche des Berichtsjahrs mit dem Vorjahr ist in der Unfallversicherung die Zahl der Policen von 144452 auf 143005 oder um 1,0 Prozent zurückgegangen, die Prämieneinnahme dagegen von $7860879 \mathscr{H}$ auf $8148734 \mathscr{N}$ oder um 3,7 Prozent gestiegen; bei der Haftpflichtversicherung ist die Zahl der Policen von 369835 auf 376285 oder um 1, 7 Prozent, die Prämieneinnahme von $8155277 \mu$ auf $8687442 \mu$ oder um 6,5 Prozent gestiegen.

Zur Beurteilung des Vermögensstandes der ausländischen Gesellschaften sind in diese Statistik die Bilanzen der Gesellschaften aufgenommen, wie sie für das ganze Geschäft der Gesellschaften (nicht nur für das deutsche Geschäft) gelten. Dabei ist möglichst nach denselben Grundsätzen und in denselben Formen vorgegangen wie 
bei den deutschen Unternehmungen; es bedarf daher hier keiner besonderen Erklärungen.

Die Bilanz ist hier nur für sieben Gesellschaften zu geben (Tabelle II 14), da die Bilanz der Basler Lebens-Versicherungs-Gesellschaft nach Gruppe I genommen ist.

Hinsichtlich der Kapitalanlagen dieser sieben Gesellschaften ist zu bemerken, daß die vier österreichischen Unternehmungen von ihrem ganzen Bestande von $61589429 \mathscr{N}$ in Grundbesitz $4335850 \mathscr{N}$ oder 7,0 Prozent, in Hypotheken $2514724 \mathscr{N}$ oder 4,1 Prozent, in Wertpapieren $50679177 \mathscr{M}$ oder 82, 3 Prozent, in Policedarlehen $3508945 \mathscr{M}$ oder 5, $;$ Prozent und in Wechseln $550733 \mathscr{N}$ oder 0,9 Prozent angelegt hatten, und daß von dem gesamten Bestande (95082 095 $\mathscr{N}$ ) der drei schweizerischen Gesellschaften $6296000 \mathscr{N}$ oder 6,6 Prozent in Grundbesitz, $28828125 \mathscr{A l}$ oder 30,3 Prozent in Hypotheken, 59928800 $\mathscr{M}$ oder 63,0 Prozent in Wertpapieren und 29170 $\mathscr{N}$ oder 0,03 Prozent in Policedarlehen angelegt waren.

Ein eingehender Vergleich des ausländischen Geschäfts der deutschen Unternehmungen mit dem deutschen Geschäfte der ausländischen Unternehmungen auf dem Gebiete der Unfall- und Haftpflichtversicherung kann zwar nicht gegeben werden; indessen verdienen doch folgende Zahlen angeführt zu werden:

Im Jahre I9 IO betrugen die Bruttoprämieneinnahmen

\begin{tabular}{|c|c|c|c|}
\hline & $\begin{array}{c}\text { insgesamt } \\
1000 \mathscr{A}\end{array}$ & $\begin{array}{c}\text { für selbst } \\
\text { ahgeschlossenc Versicherungen } \\
1000 \AA\end{array}$ & $\begin{array}{c}\text { für in Rūckdeckung } \\
\text { übernommenc Versicherungen } \\
1000 \mathscr{A}\end{array}$ \\
\hline $\begin{array}{l}\text { der deutschen Gesellschaften aus dem } \\
\text { ausländischen Geschäfte ......... }\end{array}$ & 9502 & 7527 & I 975 \\
\hline $\begin{array}{l}\text { der ausländischen Gesellschaften aus } \\
\text { dem deutschen Geschäfte } \ldots \ldots \ldots \ldots\end{array}$ & 16836 & I6 645 & 191 \\
\hline
\end{tabular}

Während also die Einnahmen an Rückversicherungsprämien bei den deutschen Gesellschaften größer gewesen sind als bei den ausländischen Unternehmungen, bleiben die Prämieneinnahmen für das unmittelbare Geschäft bei den deutschen Gesellschaften hinter den entsprechenden Einnahmen der ausländischen Anstalten beträchtlich zurück; insgesamt haben die deutschen Gesellschaften für Unfall- und Haftpflichtversicherungen rund $7334000 \mathscr{M}$ weniger an Prämien aus dem Auslande bezogen, als die ausländischen Gesellschaften aus dem Deutschen Reiche eingenommen haben. In den vorhergehenden Jahren belief sich der entsprechende Betrag auf 8334000, 8026000,7502000 , (i $706000,6056000,5573000,4430000$, $3681000 \varkappa$.

Für Versicherungsleistungen sind im Jahre igro gezahlt worden:

\begin{tabular}{|c|c|c|c|}
\hline & $\begin{array}{c}\text { insgesamt } \\
1000 \text { / }\end{array}$ & $\begin{array}{c}\text { für selbst } \\
\text { abgeschlossene Versicherungen } \\
1000 \mathrm{Al}\end{array}$ & $\begin{array}{c}\text { für in Rückdeckung } \\
\text { übernommene Versicherungeı } \\
1000 \mathscr{A}\end{array}$ \\
\hline $\begin{array}{l}\text { von den deutschen Gesellschaften für } \\
\text { ausländische Versicherungen } . . . .\end{array}$ & 5634 & 4334 & 1300 \\
\hline $\begin{array}{l}\text { von den ausländischen Gesellschaften } \\
\text { für deutsche Versicherungen ...... }\end{array}$ & 7704 & 7416 & 288 \\
\hline
\end{tabular}




\section{Gruppe III, Landwirtschaftliche Versicherung}

Für diese Gruppe kommen zur Zeit nur die Viehversicherung und die Hagelver. sicherung in Frage; sonstige landwirtschaftliche Versicherungen unterstanden im Jahre I 9 I 0 der Reichsaufsicht nicht.

\section{Viehversicherung}

Dic

Unternelsmungen

Die Viehversicherung wird im Deutschen Reiche außer von einigen größeren Unternehmungen von mehreren tausend kleinen Vereinen von lokaler Bedeutung betrieben. Davon unterliegt der Reichsaufsicht nur ein kleiner Bruchteil; dieser Bruchteil enthält wieder eine Anzahl Vereine von so untergeordneter Bedeutung, daß sich ihre eingehendere statistische Betrachtung nur in Verbindung mit der Gesamtheit aller kleinen Unternehmungen lohnen würde, vgl. Veröffentlichungen des Kaiserlichen Aufsichtsamts I91 I, S. 68 und 69 .

Die vorliegende Statistik beschränkt sich daher auf die 29 wichtigsten größeren Gegenseitigkeitsvereine, nämlich auf die in der vorjährigen Statistik behandelten 28 Vereine und die I $\$ 94$ gegründete Erste Württembergische Vieh-Versicherungs-Gesellschaft a. G. in Stuttgart sowie auf 7 sonstige Unternehmungen.

Die Gegenseitigkeitsvereine betreiben sämtlich die Viehlebensversicherung, mit Ausnahme des Vich-Versicherungs-Vereins der Viehhändler in Hannover, der sich auf die Schlachtviehversicherung beschränkt. Von den anderen Vereinen betreiben acht neben der Viehlebensversicherung auch die Schlachtviehversicherung, sechszehn noch andere Nebenarten der Viehversicherung, während einer, die Altenburger Vieh-VersicherungsGesellschaft, außer der Viehversicherung noch die Sachschäden-Haftpflichtversicherung, ein anderer, der Central-Viehversicherungs-Verein in Berlin, noch Fuhrunfallversicherung betreibt. Die übrigen Unternehmungen schließen Viehlebensversicherungen überhaupt nicht ab; sie wirken sämtlich und ausschließlich auf dem Gebiete der Schlachtviehversicherung; nur die Hamburg-Altonaer Vich-Versicherung (Gesellschaft mit beschränkter Haftung) und die Hamburger Schlachtvieh-Versicherung, Gebr. Skriver, betreiben auch die Quarantäneversicherung. Die Schlesische Schlachtvieh-Versicherung von Julius Priesemuth in Breslau betreibt auch die kurzfristige Nutzvieh- und Kuhkalbenversicherung.

Aktiengesellschaften, welche auf dem Gebiete der Viehversicherung arbeiten, waren nicht vorhanden.

Ausländische Unternehmungen waren in der Viehversicherung zum Geschäftsbetrieb im Deutschen Reiche nicht befugt.

Umfang der

Versicherung.

Prămien,

Schăden

Nach Tabelle III 1 hatten die sämtlichen in Betracht kommenden 36 Unternehmungen im Berichtsjahre Versicherungen im Betrage von 783,515 Millionen Mark; davon kommen 648,327 Millionen auf die 29 Gegenseitigkeitsvereine (mit 432,567 Millionen Mark gegen feste Prämien) und 135,188 Millionen auf die 7 anderen Unternehmer. Rückversicherungen finden sich nur bei den Gegenseitigkeitsvereinen. Von dem Gesamtbetrage der Versicherungen dieser Vereine waren 2,446 Millionen Mark in Rückdeckung übernommen und 7,455 Millionen Mark in-Rückdeckung gegeben, so daß die Vereine 
für eigene Gefahr 640,872 Millionen Mark versichert hatten. Die Rückversicherung spielt hiernach bei der Viehversicherung nur eine untergeordnete Rolle.

Das Anwachsen der Versicherungssummen in den letzten Jahren ist in der beigegebenen Tafel dargestellt.

Die Nettoprämieneinnahme aller Unternehmungen, d. h. die im Laufe des Jahres von den Versicherten eingenommenen Beträge nach Abzug der Rückversicherungsprämien, hat $12576393 \mathscr{A}$ (6878554 $/$ Vorprämien, $881902 \mathscr{A}$ Nachschußprämien, $5037014 \mathscr{N}$ feste Prämien und $221077 \mathscr{M}$ Rückversicherungsprämien, Tabelle III 5) betragen.

Gegen feste Prämien werden in der Regel abgeschlossen: die Schlachtviehversicherung und die in Tabelle III 2 unter sonstiger Viehversicherung zusammengefabten Arten, nämlich die Rückversicherung, die Viehtransport-, die Manöver-, Weide-, Stuten-, Operations-, Ausstellungs-, Quarantäneversicherung usw.

Als Schäden für eigene Rechnung ohne Regulierungskosten, aber unter Berücksichtigung des Zuwachses oder der Abnahme der Schadenreserve und unter Einschluß des Erlöses für verwertetes Vieh sind $13977530 \mathscr{M} \mathrm{zu}$ verrechnen gewesen.

Eine Statistik über die Ursachen der Entschädigungspflicht im Jahre 1910 ist in dem Geschäftsberichte des Aufsichtsamts für I 9 IO veröffentlicht, vgl. Veröffentlichungen I 9 I I S. $101 \mathrm{ff}$.

Die Ubersicht S. $48^{*}$ und $49^{*}$ zeigt, wie sich die Versicherungsprämien und die Schäden auf die verschiedenen Versicherungsmodalitäten, die Vieharten und deren Stückzahl und Wert verteilen. Sie beruht auf denselben Grundlagen wie Tabelle III 2; es sind daher auch in dieser Ubersicht die Anteile der Rückversicherer überall eingeschlossen. Die Prämien, von denen die Rabatte nicht abgesetzt sind, sind die Einnahmen im Geschäftsjahr; die Schäden sind die im Laufe des Geschäftsjahrs entstandenen Schäden. In dieser Zusammenstellung kommt beispielsweise besonders deutlich zum Ausdruck, wie sehr verschieden die Verhältnisse bei den verschiedenen Versicherungsarten liegen.

Seit dem Jahre 1905 hat der gesamte Versicherungsbestand nach Vieharten verteilt werden können; es mag deshalb hier eine kleine Ubersicht über die Stückzahl der versicherten Tiere und der zugehörigen Versicherungssummen Platz finden.

\begin{tabular}{|c|c|c|c|c|c|c|c|c|}
\hline \multirow{2}{*}{ Geschäftsjalır } & \multicolumn{3}{|c|}{ Viehlebensversicherung } & \multicolumn{2}{|c|}{ Schlachtviehversicherung } & \multicolumn{3}{|c|}{ Sonstige Viehversicherung } \\
\hline & Pferde & Rindvieh & Schweine & Rindvieh & Schweine & Pferde & Rindvieh & Schweine \\
\hline & \multicolumn{8}{|c|}{ Zahl der versicherten Ticre } \\
\hline $1905 \ldots$ & 234112 & I41 766 & 164672 & $52025^{8}$ & I 397999 & 12367 & 72802 & 231034 \\
\hline $1906 \ldots$ & 230526 & 125771 & 151648 & 557533 & I 376184 & 9285 & 79850 & 214972 \\
\hline $1907 \ldots$ & 219332 & 108938 & 178079 & 557935 & 1564674 & 8702 & 78650 & 167478 \\
\hline 1908. & 217140 & 101818 & 149353 & ') 730456 & 1) I 862845 & 10078 & 88819 & 142566 \\
\hline $1909 \ldots$ & 214183 & 92524 & I 53335 & 817252 & 1862784 & 14446 & 96703 & 159037 \\
\hline $1910 \ldots$ & 219494 & $8+100$ & 131164 & 852060 & 2027207 & 25848 & $13455^{8}$ & 246647 \\
\hline & \multicolumn{8}{|c|}{ Versicherungssummen in $1000 \mathscr{A}$} \\
\hline 1905 & 166072 & 43859 & 10316 & 154868 & 157374 & 7920 & 25147 & $24 \mathrm{I}_{42}$ \\
\hline $1906 \ldots$ & 167942 & 40593 & 10108 & 175714 & 163398 & 7683 & 29003 & 22061 \\
\hline $1907 \ldots$ & 166369 & 36491 & 9849 & 1 72369 & 170178 & 7517 & 27 I15 & 17746 \\
\hline $1908 \ldots$ & 166283 & 34417 & 7677 & 1) 199761 & 1) $20226 \mathrm{I}$ & 8923 & 28769 & 12273 \\
\hline $1909 \ldots$ & 167813 & $313^{81}$ & 7292 & 221466 & 216337 & 12677 & 30326 & 15050 \\
\hline $1910 \ldots \ldots \ldots$ & 175001 & 29052 & 6432 & 244852 & 232181 & 19769 & 48315 & 25980 \\
\hline
\end{tabular}




\begin{tabular}{|c|c|c|c|c|c|c|}
\hline $\mathrm{Viehar}$ & Stückzahl & $\begin{array}{c}\text { Ver- } \\
\text { sicherungs- } \\
\text { summen } \\
\\
.4 \\
\end{array}$ & Prämien & $\begin{array}{l}\text { Zahl der } \\
\text { Schäden }\end{array}$ & $\begin{array}{l}\text { Betrag der } \\
\text { Schäden } \\
\text { einschl. } \\
\text { Erlös } \\
\\
\qquad \mathscr{H} \\
6\end{array}$ & $\begin{array}{c}\text { Erlüs } \\
\mathscr{H}\end{array}$ \\
\hline $\mathbf{I}$ & 2 & 3 & 4 & 5 & & \\
\hline & & & & & \multicolumn{2}{|c|}{ Viehversicherung } \\
\hline Pferde (Maultiere, Esel) ..... & 245353 & 194770771 & 7224102 & 13405 & 6182579 & 874891 \\
\hline Rindvieh $\ldots \ldots \ldots \ldots \ldots \ldots$ & 1070718 & 322219769 & 4007940 & 71532 & 6103669 & 2844 I96 \\
\hline Schafe und Ziegen .......... & 46269 & I 838340 & 23167 & I 708 & 18213 & 2923 \\
\hline Schweine................ & 2405 or 8 & 264592747 & 1572913 & 61153 & I 866096 & 764401 \\
\hline Sonstiges Kleinvieh.......... & 1179 & 93617 & 6105 & 34 & 1480 & 24 \\
\hline Zusammen .... & 3768537 & 783515244 & 12834227 & 147832 & 14172037 & 4486435 \\
\hline
\end{tabular}

Viehlebensversicherung

\begin{tabular}{|c|c|c|c|c|c|c|}
\hline Pferde (Maultiere, Esel) ..... & 219494 & 175001153 & 6779237 & I 2365 & 596 I 861 & 864562 \\
\hline Rindvieh $\ldots \ldots \ldots \ldots \ldots \ldots$ & 84100 & 29052274 & 738697 & 3211 & 892160 & 266057 \\
\hline Schafe und Ziegen ......... & 4837 & 180316 & 9869 & 307 & 9269 & 712 \\
\hline Schweine............... & I3I 164 & 6432314 & 199263 & 3786 & I 76804 & $2394 \mathrm{I}$ \\
\hline Sonstiges Kleinvieh......... & 487 & 68120 & 5147 & 28 & I 229 & 24 \\
\hline Zusammen & 440082 & 210734177 & 7732213 & 19697 & 7041323 & 1155296 \\
\hline
\end{tabular}

Schlachtviehversicherung

\begin{tabular}{|c|c|c|c|c|c|c|}
\hline Pferde (Maultiere, Esel) ..... & II & 1020 & 44 & - & - & - \\
\hline Rindvieh $\ldots \ldots \ldots \ldots \ldots \ldots$ & 852060 & 244852466 & 2912706 & 65901 & 4604663 & 2221905 \\
\hline Schafe und Ziegen .......... & 39467 & I 489 I $5^{2}$ & II 429 & I $3^{86}$ & $8 \circ 67$ & 2208 \\
\hline Schweine..$\ldots \ldots \ldots \ldots \ldots$ & 2027207 & $232 \quad 180883$ & I 288074 & 55024 & I 582294 & 712574 \\
\hline Sonstiges Kleinvieh......... & I & 9 & I & - & - & 一 \\
\hline Zusammen .... & 2918746 & 478523530 & 4212254 & 122311 & 6195024 & 2936687 \\
\hline
\end{tabular}

\section{Sonstige Viehversicherung}

\begin{tabular}{|c|c|c|c|c|c|c|}
\hline Pferde (Maultiere, Esel) ..... & 25848 & 19768598 & 444821 & 1040 & 220718 & 10329 \\
\hline Rindvieh $\ldots \ldots \ldots \ldots \ldots \ldots$ & $13455^{8}$ & 48315029 & 356537 & 2420 & 606846 & 356234 \\
\hline Schafe und Ziegen .......... & 1965 & 168872 & I 869 & 15 & 877 & \\
\hline Schweine..$\ldots \ldots \ldots \ldots \ldots$ & 246647 & 25979550 & 85576 & 2343 & 106́ 998 & 27886 \\
\hline Sonstiges Kleinvieh.......... & 691 & 25488 & 957 & 6 & $25 \mathrm{I}$ & - \\
\hline Zusammen .... & 409709 & 94257537 & 889760 & 5824 & 935690 & 394452 \\
\hline
\end{tabular}

Anmerkung: Ein - bedeutet, daß dic beobachteten Fälle weniger als $1 / 2 \% 10$ ansmachen. 
Viehrersicherung

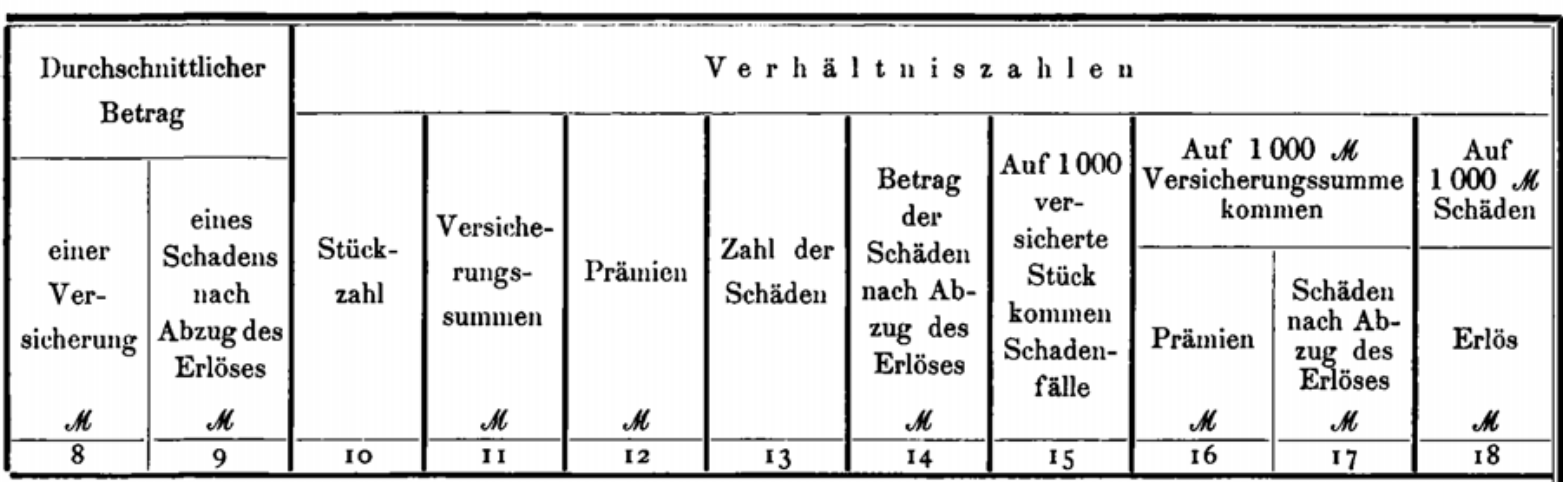

insgesamt

\begin{tabular}{|r|r|r|r|r|r|r|r|r|r|r|}
794 & 396 & 65 & 249 & 563 & 91 & 548 & 55 & 37 & 27 & 142 \\
301 & 46 & 284 & 411 & 312 & 484 & 336 & 67 & 12 & 10 & 466 \\
40 & 9 & 12 & 2 & 2 & 11 & 2 & 37 & 13 & 8 & 160 \\
110 & 18 & 638 & 338 & 123 & 414 & 114 & 25 & 6 & 4 & 410 \\
79 & 43 & 1 &. &. &. &. & 29 & 65 & 16 & 16 \\
\hline 208 & 66 & 1000 & 1000 & 1000 & 1000 & 1000 & 39 & 16 & 12 & 317
\end{tabular}

(28 Gegenseitigkeitsvereine)

\begin{tabular}{|r|r|r|r|r|r|r|r|r|r|r|}
797 & 412 & 499 & 830 & 877 & 628 & 866 & 56 & 39 & 29 & 145 \\
345 & 195 & 191 & 138 & 95 & 163 & 106 & 38 & 25 & 22 & 298 \\
37 & 28 & 11 & 1 & 1 & 16 & 2 & 63 & 55 & 47 & 77 \\
49 & 40 & 298 & 31 & 26 & 192 & 26 & 29 & 31 & 24 & 135 \\
140 & 43 & 1 &. & 1 & 1 &. & 57 & 76 & 18 & 20 \\
\hline 479 & 299 & 1000 & 1000 & 1000 & 1000 & 1000 & 45 & 37 & 28 & 164 \\
& & & & & & & &
\end{tabular}

(16 Unternehmungen)

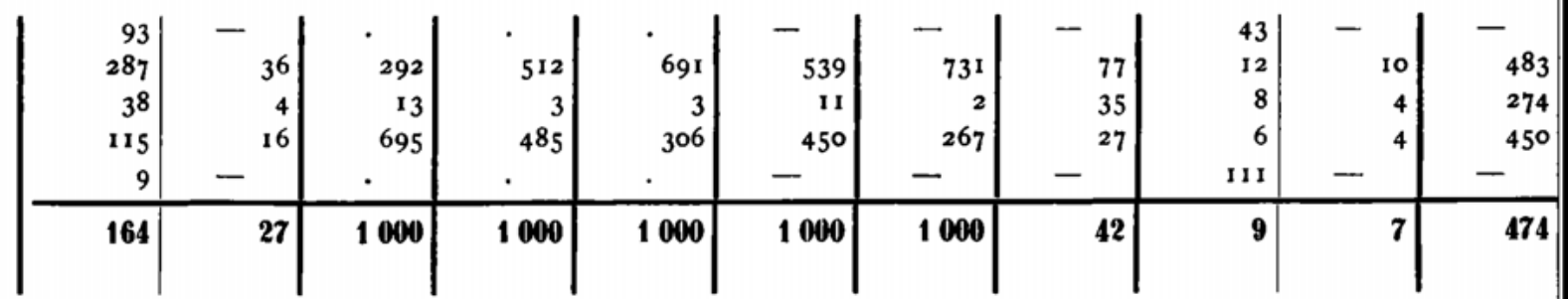

(19 Unternehmungen)

\begin{tabular}{|r|r|r|r|r|r|r|r|r|r|r|}
765 & 202 & 63 & 210 & 500 & 179 & 389 & 40 & 23 & 11 & 47 \\
359 & 104 & 328 & 512 & 401 & 415 & 463 & 18 & 7 & 5 & 587 \\
86 & 58 & 5 & 2 & 2 & 3 & 2 & 8 & 11 & 5 & 3 \\
105 & 34 & 602 & 276 & 96 & 402 & 146 & 9 & 3 & 3 & 261 \\
37 & 42 & 2 &. & 10 & 1 &. & 9 & 38 & 10 \\
\hline 230 & 93 & 1000 & 1000 & 1090 & 1000 & 1000 & 14 & 9 & 6 & 422 \\
& & & & & & & &
\end{tabular}


Vergleich mit dem Vorjahre Stellen nicht der Betrag der überhaupt in Kraft gewesenen Versicherungen, sondern der Betrag der am Ende des Jahres laufenden Versicherungen eingestellt werden mußte. Bei den Gegenseitigkeitsvereinen haben die Versicherungssummen um 60,281 Millionen Mark oder 10,3 Prozent, die Prämien um 0,184 Millionen oder 1,6 Prozent und die Schadenbeträge (ohne Abzug des Erlöses) um 0,183 Millionen oder 1,6 Prozent zugenommen. Der Vergleich von 1909 mit 1908 hatte nach der vorjährigen Statistik bei den Versicherungssummen eine Zunahme um 41,667 Millionen Mark oder 7,6 Prozent, bei den Prämien um 0,433 Millionen oder 4,0 Prozent und bei den Schäden um 0,707 Millionen oder 6,4 Prozent ergeben.

Bei den sonstigen Unternehmungen haben die Versicherungssummen, Prämien und Schäden ebenfalls zugenommen, und zwar die Versicherungssummen um 17,496 Millionen oder 15,0 Prozent, die Prämien um 0,240 Millionen oder 24,4 Prozent und die Schadenzahlungen um 0,796 Millionen oder 65,4 Prozent gegen $0,4,2,8$, und 3,1 Prozent im Vorjahre.

Die Gewinn- und Verlustrechnung (Tabelle III 4) stellt nur das Ergebnis des Berichtsjahrs dar; der Gewinnvortrag aus dem Vorjahr ist fortgelassen, auch sind die Prämien- und Schadenreserven nicht mit ihrem ganzen Betrag am Anfange des Jahres in Einnahme und am Schlusse des Jahres in Ausgabe nachgewiesen, sondern es sind nur die Änderungen, welche der Betrieb des Jahres zur Folge hatte, verrechnet (vgl. Tabelle III 5).

Die gesamte Prämieneinnahme hat im Jahre ı 9 Iо $12797470 \mathscr{M}$ betragen. Wie sich dieser Betrag auf Vorprämien, Nachschußprämien und feste Prämien verteilt, ist bereits oben angegeben; es braucht hier nur noch hinzugefügt zu werden, daß die Nachschüsse 12,8 Prozent der Vorprämien aller Vereine und 38,2 Prozent der Vorprämien derjenigen Vereine ausgemacht haben, welche im Berichtsjahr überhaupt Nachschüsse erhoben haben. Verrechnet man hierauf die Prämienüberträge und die Rückversicherungsprämien, so erhält man die in die Betriebsrechnung eingestellte Prämieneinnahme. Die von einigen Vereinen durch die Gewinn- und Verlustrechnung geführten "Nachschußversicherungsprämien " oder "Nachschußrückversicherungsprämien " sind hier fortgelassen; es handelt sich nämlich um Prämien für eine Nebenversicherung gegen etwaigen Nachschuß, die der Verein von seinen Mitgliedern einzieht, um sie zumeist vollständig an andere Versicherungsgesellschaften abzuführen, also um durchlaufende Posten, die den Geschäftsbetrieb nicht weiter berühren. Die Leistungen der Versicherer aus diesen Versicherungen sind bei den Nachschüssen eingestellt.

Nebenleistungen der Versicherten kommen, außer bei der Schlesischen Schlachtvieh-Versicherung von Julius Priesemuth, nur bei den Gegenseitigkeitsvereinen vor; sie haben bei letzteren im Jahre $1910384095 \mu$, das sind 3,3 Prozent der ganzen Prämieneinnahme oder 5,6 Prozent der Vorprämien betragen; dabei ist zu bemerken, daß bei den meisten Versicherungsgesellschaften als satzungsmäßige Leistung der Mitglieder zum Reservefonds bestimmte Prozente der Vorprämie verrechnet und als Teile der Vorprämie nachgewiesen werden, also nicht bei den Nebenleistungen erscheinen.

Der Erlös aus verwertetem Vieh belief sich auf $4491951 \mathscr{M}$, d. h. 32,1 $\mathscr{N}$ von je $100 \mathscr{N}$ Schäden für eigene Rechnung. Hierbei ist zu beachten, daß zum Teil nicht 
nur der wirklich erzielte Erlös oder der bedingungsgemäß gestattete Mindesterlös in Rechnung gestellt ist, sondern auch vielfach, insbesondere bei Versicherungen gegen Minderwert, der Unterschied zwischen Versicherungssumme und gezahltem Schadenbetrage angerechnet ist.

An Regulierungskosten wurden $292301 \mathscr{M}$ oder $2,1 \mathscr{N}$ auf je $100 \mathscr{M}$ Schäden einschließlich Erlös gezahlt; an eigenen Verwaltungskosten und Steuern wurden $2923949 \mathscr{N}$ oder 23,3 Prozent der Prämien für eigene Rechnung (Tabelle III 5 Sp. 8) verausgabt.

Aus der Bilanz für Ende I9ıo, die nur für die Gegenseitigkeitsvereine gegeben Die Bilanz wird, mögen folgende Posten angeführt werden:

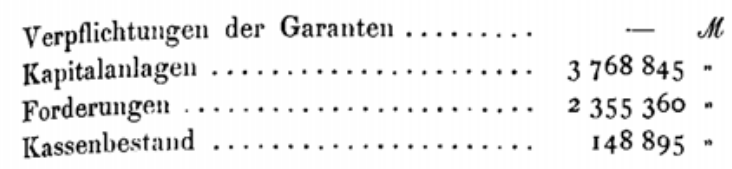

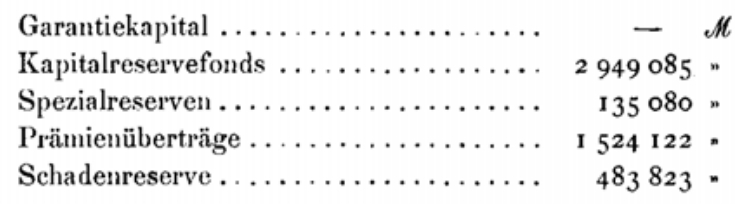

Schadenreserve................ 483823 .

Die Kapitalanlagen bestehen zu 76,3 Prozent in Wertpapieren, zu 7,2 Prozent in Grundbesitz, zu 16,3 Prozent in Hypotheken und zu 0,2 Prozent in Darlehen an öffentliche Körperschaften. Im Vergleiche mit dem Bestand am Ende des Vorjahrs waren im Besitze der Gegenseitigkeitsvereine Ende I 9 Io vorhanden an Hypotheken $76821 \mathscr{N}$, an Wertpapieren $230047 \mathscr{M}$ (dem Nennwert nach rund $241000 \mathscr{C}$ ) mehr, dagegen an Grundbesitz $5787 \mathscr{N}$ weniger. Die Forderungen bestehen bei der Gesamtheit zu 63,6 Prozent in Rückständen der Versicherten (einschl. der noch nicht gezahlten Nachschüsse); ins Gewicht fallen bei einigen Gesellschaften auch die Ausstände bei Agenten.

Die Kapitalreservefonds haben gegen die Bilanz für Fnde rgog um $49637 \mathscr{M}$ oder um 1,7 Prozent zugenommen; die Spezialreserven vermehrten sich um $22671 \mathscr{M}$ oder 20,2 Prozent.

Die vorstehenden Angaben beziehen sich sämtlich auf den gesamten Geschäftsbetrieb der genannten deutschen Viehversicherungsunternehmungen. Zur Beurteilung der Ausdehnung und des Umfanges des darin enthaltenen ausländischen Geschäfts mögen folgende Nachrichten dienen.

Im Jahre I9 Io haben wie in den Vorjahren nur 5 Versicherungsvereine auch im Auslande Geschäfte abgeschlossen, davon je einer in Belgien, Luxemburg, Norwegen, Schweden und in der Schweiz und je zwei in Dänemark und Österreich. Diese Vereine hatten am Ende des Jahres im Ausland eine Summe von $3630793 \mathscr{N}$ versichert. An Prämien haben sie im Berichtsjahr $176004 \mathscr{N}$ eingenommen, an Schäden sind $129579 \mathscr{N}$ gezahlt. Das ausländische Geschäft war also in der Viehversicherung nur unbedeutend; es betrug 4,6 vom Tausend der gesamten Versicherungssumme, 14,0 vom Tausend der Prämieneinnahme und 9,3 vom Tausend der Schäden der Vereine. 


\section{Hagelversicherung}

Die Unternehmungen

Von den im Deutschen Reiche im Jahre r9ro vorhanden gewesenen Hagelver. sicherungsunternehmungen unterstanden dem Aufsichtsamte 5 Aktiengesellschaften und 15 Gegenseitigkeitsvereine. Davon sind in diese Statistik außer den Aktiengesellschaften 12 Vereine aufgenommen; die übrigen drei Vereine sind von ganz lokalem Charakter und geringer Bedeutung, so daß von ihnen nicht wohl alle Angaben, welche diese Statistik bringt, gefordert werden können.

Drei von den hier behandelten 12 Gegenseitigkeitsvereinen betreiben auch die Feuerversicherung, nämlich die Greifswalder, die Mecklenburgische und die Schwedter Hagel- und Feuer-Versicherungs-Gesellschaft; eine, die Greifswalder, betreibt auch die Baulastversicherung. Alle drei halten aber die Versicherungszweige in ihren Büchern getrennt und geben eine vollständige Gewinn- und Verlustrechnung und Bilanz für jeden Zweig. Diese vollständige Trennung ist auch hier beibehalten worden, so daß sich die hier für diese Gesellschaften gegebenen Zahlen lediglich auf die Hagelversicherung beziehen, wie die für dieselben Gesellschaften bei Gruppe IV gegebenen Zahlen lediglich das Feuerversicherungsgeschäft betreffen.

Ausländische Hagelversicherungsgesellschaften haben, soweit bekannt, im Jahre I 910 im Deutschen Reiche nicht gearbeitet.

Der Umfang des Geschäfts im Berichtsjahre wird durch folgende Zahlen bezeichnet. Es waren insgesamt 2 879,189 Millionen Mark auf 464185 selbst abgeschlossene Versicherungen versichert oder im Durchschnitt $6203 \mathscr{N}$ auf eine Police.

Hierin sind 159,597 Millionen Mark enthalten, die zu 20212 Kollektiv- oder Gemeindeversicherungen gehören, welche in der vorstehenden Zahl der Versicherungen je nur als eine Versicherung gezählt sind, aber 178043 Teilnehmer hatten. Die durch. schnittlich von einem Versicherten versicherte Summe stellt sich demnach bei der Gesamtheit auf $4629 \mathscr{M}$ und bei den Gemeinde- und Kollektivversicherungen allein auf $896 \mathscr{M}$.

Von den Versicherungen kommen auf die Aktiengesellschaften 944,192 Millionen Mark und 138218 Policen oder 6831 $\mathscr{M}$ im Durchschnitt für die Police und auf die Gegenseitigkeitsvereine 1934,997 Millionen Mark und 325967 Versicherungen oder $5936 \mathscr{N}$ im Durchschnitt für die Police.

Die Rückversicherung hatte keine große Bedeutung. Es hatten in Rückdeckung übernommen fünf Aktiengesellschaften zusammen 70,033 Millionen Mark und in Rückdeckung gegeben fünf Gesellschaften 60,290 Millionen Mark, so daß die Aktiengesellschaften zusammen von 1 014,225 Millionen Mark überhaupt übernommener Versicherungen 953,935 Millionen für eigene Rechnung behalten haben. Von den Gegenseitigkeitsvereinen haben vier 11,468 Millionen Mark in Rückdeckung übernommen und 11,469 Millionen Mark in Rückdeckung gegeben, so daß bei diesen Anstalten von 1946,465 Millionen Mark überhaupt übernommener Versicherungen 1934,996 Millionen für eigene Rechnung blieben.

Die Nettoprämieneinnahmen, d. h. die im Laufe des Jahres von den Versicherten eingenommenen Prämien, vermindert um die ausgegebenen Rückversicherungsprämien, haben insgesamt betragen $46425278 \mathscr{M}$, wovon $10961300 \mathscr{N}$ auf die Aktiengesellschaften und $35463978 \mathscr{N}$ auf die Gegenseitigkeitsvereine entfielen (Tabelle III 11).

Als Schäden für eigene Rechnung, ohne die Ermittelungskosten, aber unter Berücksichtigung der Änderung der Schadenreserve am Anfange des Jahres gegen die 
Schadenreserve am Schlusse des Jahres (vgl. Tabellen III 11 und 13), sind insgesamt $41200664 \mathscr{M}$ zu verrechnen gewesen: $8559067 \mathscr{M}$ bei den Aktiengesellschaften und 32 $641597, \mathscr{N}$ bei den Gegenseitigkeitsvereinen. Als jedenfalls nicht zu kleine Zahl der Schäden (weil bei drei Gesellschaften nicht die Zahl der Schäden, sondern die Zahl der Anmeldungen angegeben ist) darf für die Gesamtheit 97673 angenommen werden, nämlich 34591 für die Aktiengesellschaften und 63082 für die Gegenseitigkeitsvereine. Es kommen also auf den Schadenfall im Durchschnitte mindestens: bei der Gesamtheit $422 \mathscr{N}$, bei den Aktiengesellschaften $247 \mathscr{N}$ und bei den Gegenseitigkeitsvereinen $517 \mathscr{N}$.

Als Entschädigungsabzüge sind insgesamt $3432296 \mathscr{M}$ einbehalten worden, $525305 \mathscr{N}$ von den Aktiengesellschaften und $2906991 \mathscr{M}$ von den Gegenseitigkeitsvereinen. Die Abzüge bestanden bei den Aktiengesellschaften ausschließlich in Anteilen der Geschädigten an den Ermittelungskosten; von den Abzügen der Gegenseitigkeitsvereine waren $1150100 \%$ für Ermittelungskosten, $1732587 \mathscr{N}$ für die Reservefonds und $24304 \mathscr{H}$ für andere $Z$ wecke bestimmt.

Die Prämienüberträge und die Schadenreserve spielen bei der Hagelversicherung naturgemäß nur eine ganz untergeordnete Rolle (vgl. Tabelle III I 3); man kann daher von diesen Rückstellungen absehen und folgende Zahlen als zutreffend annehmen:

Von $1000 \mathscr{N}$ Prämien wurden für Schadenzahlungen nach Verrechnung der Entschädigungsabzüge verwendet:

\begin{tabular}{|c|c|c|c|c|c|}
\hline im Jahre.... & 1906 & 1907 & 1908 & 1909 & I910 \\
\hline von allen Unt & $901 \mathscr{H}$ & $868 \mathscr{N}$ & $928 \mathscr{H}$ & $516 \mathscr{N}$ & $887 \mathscr{M}$ \\
\hline " den Aktienge & $78 \mathrm{r}$ & $866 n$ & I 097 . & $218 \cdots$ & 781 \\
\hline . $\quad$ Gegenseitigkeitsvereinen ................ & $917 n$ & 868 . & $875 \cdots$ & $736 \cdot$ & $920 \times$ \\
\hline
\end{tabular}

Auf $1000 \mathscr{M}$ Versicherungssumme kamen:

\begin{tabular}{|c|c|c|c|c|c|c|c|}
\hline Präm & bei der & Ges: & $13,20 \mathscr{N}$ & $13,45 \mathscr{M}$ & 16,01 $\mathscr{N}$ & $8,68 \mathscr{M}$ & $\mathrm{1} 6, \mathrm{1} 2 \mathscr{H}$ \\
\hline - & der & ellschaften & 9,75 & 9,83 . & 9,70 . & $9,67=$ & II 6 I \\
\hline n & - & Gegenseitigkeitsvereinen... & 15,78 . & 15,93 & 20,18 " & 8,07 " & 18,33 . \\
\hline häden & bei & Gesa & II, 89 . & $11,67 \cdot$ & 14,89 " & 4,48 n & 14,31 \\
\hline - & " den & Aktiengesell & $8,46 \cdots$ & $8,52 \times$ & Io,64 n & $2,10 \cdots$ & 9,06 \\
\hline 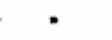 & " & $\cdots$ & $14,47 "$ & I $3,83_{3}$ " & 17,66 . & 5,94 & I 6,87 \\
\hline
\end{tabular}

Eine Vergleichung des Berichtsjahrs mit dem Vorjahre führt zu folgenden Er- vergleich gebnissen (Tabelle III 11).

Die Zahl der Versicherungen hat bei der Gesamtheit der Unternehmungen um 23360 oder 4,3 Prozent zugenommen, im besonderen bei den Aktiengesellschaften um 3528 oder 1,5 Prozent und bei den Gegenseitigkeitsvereinen um 19832 oder 6,4 Prozent. Die Versicherungssummen sind bei der (xesamtheit der Unternehmungen um 31798 Millionen Mark oder 1,1 Prozent gestiegen. Sie sind bei den Aktiengesellschaften um 102,796 Millionen Mark oder 9,2 Prozent gefallen und bei den Gegenseitigkeitsvereinen um 134,594 Millionen Mark oder 7,4 Prozent gestiegen. Die Nettoprämieneinnahme (einschl. Nachschüsse) ist im Berichtsjahre gegenüber dem Vorjahre bei den Aktiengesellschaften um $318400 \mathscr{M}$ oder 3,0 Prozent, bei den Gegenseitigkeitsvereinen um $20994074 \mathscr{N}$ oder 145,1 Prozent gestiegen. Die Schäden für eigene Rechnung sind bei den Aktiengesellschaften um $6243526 \mathscr{M}$ oder 269,6 Prozent, bei den Gegenseitigkeitsvereinen um $21989805 \mathscr{N}$ oder 206,4 Prozent höher gewesen als im Vorjahre.

Die in Tabelle III 12 gegebene Gewinn- und Verlustrechnung stellt nur das Ergebnis des Berichtsjahrs dar; der Gewinnvortrag aus dem Vorjahr ist fortgelassen; auch sind die sonstigen Uberträge nicht mit ihrem vollen Betrage zu Anfang des Jahres 
in Einnahme und am Schlusse des Jahres in Ausgabe nachgewiesen, sondern es sind nur die Änderungen, welche der Betrieb des Geschäftsjahrs zur Folge hatte, verrechnet (vgl. Tabelle III 13).

Die Prämien haben bei den Aktiengesellschaften $11602745 \mathscr{N}$ betragen, wovon für Rückversicherung $641536 \mathscr{N}$ wieder ausgegeben wurden. Die Gegenseitigkeitsvereine haben $14747163 \mathscr{N}$ Vorprämie und $20794763 \mathscr{N}$ Nachschußprämie erhoben; für Rückversicherung haben sie $83333 \mathscr{M}$ wieder ausgegeben; die Nachschußprämie machte 141,0 Prozent von der Vorprämie aus, oder wenn man die Greif'swalder, Schles. wig-Holsteiner in Kiel, Neubrandenburger, den Holsteinischen H.V.V. in Neustadt und die Oldenburger H.V.G. ausschließt, weil sie die Beiträge durch Umlage nach Bedarf auf bringen, 168,9 Prozent. Die von mehreren Gegenseitigkeitsvereinen durch die Gewinn- und Verlustrechnung geführten "Rückversicherungsprämien " sind hier fortgelassen; es handelt sich nämlich um Prämien für eine Nebenversicherung gegen etwaigen Nachschuß, die der Verein von seinen Mitgliedern einzieht, um sie meist vollständig an andere Versicherungsgesellschaften abzuführen, also um durchlaufende Posten, die den Geschäftsbetrieb nicht weiter berühren. Die Leistungen der Versicherer aus diesen Versicherungen sind bei den Nachschüssen eingestellt.

An Nebenleistungen hatten die Versicherten zu zahlen bei den Aktiengesellschaften $117206 \mathscr{N}$, das sind 1,0 Prozent der Prämien, und bei den Gegenseitigkeitsvereinen $1888263 \mathscr{N}$, das sind 5,3 Prozent der gesamten Prämien oder 12,8 Prozent der Vorprämien. Die Nebenleistungen bestehen bei den Gegenseitigkeitsvereinen zum größten Teile $(1579960 \mathscr{C})$ in satzungsmäßigen Beiträgen zum Reservefonds, die entweder nach der Höhe der Vorprämie oder der Entschädigung bemessen werden; setzt man diese ab, so bleiben $308303 \mathscr{M}$, das sind 0,9 Prozent der gesamten Prämien oder 2,1 Prozent der Vorprämien. Die von einigen Gegenseitigkeitsvereinen erhobenen Legegelder (Sicherheitsleistungen) sind hier außer acht gelassen.

An Schadenermittelungskosten sind ohne die oben erwähnten Abzüge bei allen Unternehmungen $1770990 \mathscr{N}$ oder $4,3 \mathscr{N}$ auf je $100 \mathscr{M}$ Schadenzahlung, bei den Aktiengesellschaften $645591 \mathscr{N}$ oder $7,5 \mathscr{M}$ und bei den Gegenseitigkeitsvereinen $1125399 \mathscr{M}$ oder $3,4 \mathscr{N}$ auf je $100 \mathscr{N}$ Schäden entstanden.

Das Geschäft des Jahres I 9 I 0 hat 3 Aktiengesellschaften einen Gewinn von zusammen $787027 \mathscr{N}$ und 2 Aktiengesellschaften einen Verlust von insgesamt $561638 \mathscr{N}$ gebracht. Das gilt von dem gesamten Geschäfte. Scheidet man bei den einzelnen Gesellschaften das Ergebnis der Vermögensverwaltung aus, so zeigt das eigentliche Versicherungsgeschäft des Berichtsjahres ein noch viel ungünstigeres Bild: der Gewinn jener 3 Gesellschaften ermäßigt sich auf $379558 \mathscr{M}$, und der Verlust der beiden andern Gesellschaften steigt auf $753793 \mathscr{M}$. Die Gegenseitigkeitsvereine decken ihren Verlust meist schon vor $\Lambda$ bschluß der Rechnung aus dem Reservefonds oder durch Nachschüsse (vgl. "Entwickelung “ S. 39); darüber hinaus haben zwei Vereine für das Berichtsjahr einen Gewinn von zusammen $63564 \mathscr{N}$ und ein Verein einen Verlust von $50969 \mathscr{N}$ nachgewiesen. Daß aber auch für sie dieses Jahr sehr ungünstig gewesen ist, zeigen die ungewöhnlich hohen Nachschüsse, die erforderlich geworden sind. und für die Gegenseitigkeitsvereine zusammen mit $7642535 \mathscr{M}$ abschließt, mögen folgende Posten angeführt werden: 
Aktiva

Verpflichtungen der Aktionärc oder Garanten

Kapitalanlagen . . . . . . . . . . . . . . . .

Forderungen $\ldots \ldots \ldots \ldots \ldots \ldots \ldots \ldots \ldots$

Kassenbestand $\ldots \ldots \ldots \ldots \ldots \ldots \ldots \ldots \ldots$.

Passiva

Aktien- oder Garantiekapital............

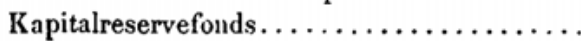

Spezialreserven.$\ldots \ldots \ldots \ldots \ldots \ldots \ldots \ldots$

Schadenreserven .....................
5 Aktiengesellschaften

$18022800 \mathscr{M}$

10981264 .

2225320 . 73768 .

$28528500 \mathscr{M}$

34 I 969 "

4572898 .

10967 .
12 Gegenseitigkeitsvereine

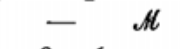

4482062 "

3025248 .

122888 .

Uber die Kapitalanlagen gibt Tabelle III 15 folgende Aufschlüsse. Von $1000 \mathscr{A}$ Kapitalanlagen kamen Ende i 9 Io auf:

\begin{tabular}{|c|c|c|c|c|c|c|c|}
\hline & $\begin{array}{c}\text { Grund- } \\
\text { besitz }\end{array}$ & $\begin{array}{l}\text { Hypo- } \\
\text { theken }\end{array}$ & $\begin{array}{c}\text { Darlehen } \\
\text { an öffentliche } \\
\text { Körnerschaften }\end{array}$ & $\begin{array}{c}\text { Darlehen } \\
\text { auf } \\
\text { Wertpapiere }\end{array}$ & $\begin{array}{c}\text { Wert- } \\
\text { papiere }\end{array}$ & Wechsel & $\begin{array}{l}\text { Sonstige } \\
\text { Anlagen }\end{array}$ \\
\hline ellschaften ......... & $28 \mathscr{M}$ & $188 \mathscr{A}$ & $-\mathscr{H}$ & $18 \mathscr{H}$ & $735, M$ & $31 \cdot x$ & $-\mathscr{M}$ \\
\hline en Gegenseitigkeitsvereinen ...... & $24 "$ & 270 & 3. & $-\cdot$ & $596 \cdots$ & $90 \%$ & $17 n$ \\
\hline
\end{tabular}

Im Vergleiche mit der Bilanz des Vorjahrs hat abgenommen der Grundbesitz um $3047 \mathscr{M}$, dagegen zugenommen der Hypothekenbestand um $323800 \mathscr{N}$ und der Buchwert der Wertpapiere um $440438 \mathscr{M}$; die Darlehen auf Wertpapiere sind unverändert geblieben.

Uber die Bedeutung des ausländischen Geschäfts der deutschen Hagelversicherungsunternehmungen, das in den vorstehenden Zahlen überall eingeschlossen ist, sind hier folgende Angaben zu machen:

Im ganzen haben vier Aktiengesellschaften und vier Gegenseitigkeitsvereine auch in Auslande Geschäfte betrieben. In Frankreich, Ungarn und der Schweiz war je eine Gesellschaft tätig, in Rußland und in Luxemburg arbeiteten je zwei, in den Niederlanden vier und in Österreich fünf Anstalten. Die Versicherungssumme belief sich auf 19,517 Millionen Mark oder 6,6 vom Tausend der von allen Unternehmungen überhaupt versicherten Summe; die durchschnittliche Höhe einer selbst abgeschlossenen ausländischen Versicherung stellte sich auf $5016 \mathscr{M}$. Die Bruttoprämieneinnahme aus dem ausländischen Geschäfte betrug $378955 \mathscr{M}$ oder 8,0 vom Tausend der gesamten Bruttoprämien; die Schäden betrugen $300781 \mathscr{M}$ oder 7,3 vom Tausend der gesamten Schäden.

\section{Gruppe IV, Feuerversicherung sowie die Versicherungen gegen Sturmschäden, Wasserleitungsschäden und Einbruchdiebstahl}

Von den deutschen Unternehmungen, die auf diesen Gebieten arbeiteten, unterstanden im Jahre I9 IO der Reichsaufsicht 45 Aktiengesellschaften und 32 Gegenseitigkeitsvereine. Diese Unternehmungen stellen aber das private Feuerversicherungsgeschäft in deutscher Hand nicht vollständig dar; es gibt vielmehr noch zahlreiche Vereine, welche wegen ihrer Beschränkung auf das Gebiet eines Bundesstaats der Aufsicht der Landesbehörden unterstehen oder welche gemäß $\S 3$ Abs. 2 des Versicherungsaufsichtsgesetzes der Landesaufsicht überwiesen worden sind.

Von den 32 unter Reichsaufsicht stehenden Feuerversicherungs-Gegenseitigkeitsvereinen sind 20 in den Tabellen ausführlich behandelt. Die Mehrzahl dieser Vereine (13) 
beschränkt sich auf die Feuerversicherung, weitere 4, nämlich der Versicherungsverein Deutscher Eisenbahnbediensteten a. G. in Berlin, die Feuer- und Einbruchdiebstahl-Versicherungsanstalt des Verbandes Deutscher Beamtenvereine a. G. in Berlin, die Landwirt. schaftliche Feuerversicherungs-Genossenschaft im Königreich Sachsen zu Dresden und die Concordia, Hannoversche Feuer-Versicherungs-Gesellschaft aufGegenseitigkeit in Hannover, betreiben daneben die Versicherung gegen Einbruchdiebstahl; die übrigen 3, die Hagelund Feuer-Versicherungsgesellschaft auf Gegenseitigkeit zu Greifswald, die Mecklenburgische Hagel- und Feuer-Versicherungs-Gesellschaft auf Gegenseitigkeit in Neubrandenburg und die Schwedter Hagel- und Feuer-Versicherungs-Gesellschaft auf Gegenseitigkeit, haben sich auch in der Hagelversicherung betätigt; die Greifswalder Gesellschaft betreibt außerdem noch die Baulastversicherung. Diese 3 Gesellschaften führen aber - wie bereits bei Gruppe III erwähnt - die verschiedenen Versicherungszweige vollständig von einander getrennt, so daß hier nur auf das Feuerversicherungsgeschäft eingegangen zu werden braucht.

$\mathrm{Zu}$ den in der vorjährigen Statistik bei Gruppe IV bearbeiteten 41 Aktiengesellschaften sind die Deutsche Reform-Versicherungsbank, Aktiengesellschaft in Berlin, die Rheinische Feuerversicherungs-Aktiengesellschaft in Cöln, die Düsseldorfer Feuerversicherungs-Aktiengesellschaft in Düsseldorf und die Hansa, Allgemeine VersicherungsAktiengesellschaft in Hamburg hinzugekommen, die im Berichtsjahre den Betrieb der Feuerversicherung, der Wasserleitungsschädenversicherung und der Einbruchdiebstahlversicherung aufgenommen haben. Die Industrie Versicherungs-Aktiengesellschaft in Berlin hat ihre Firma in Securitas, Feuer-Versicherungs-Aktiengesellschaft in Berlin geändert. - Von diesen 45 Gesellschaften betrieben 33 die Feuerversicherung neben anderen Zweigen, 3 die Feuerversicherung als alleiniges Geschäft; die übrigen 9 befaßten sich nicht mit der Feuerversicherung, sondern - soweit die Versicherungszweige der Gruppe IV in Betracht kommen - nur mit der Sturmschädenversicherung und der Wasserleitungsschädenversicherung und besonders mit der Einbruchdiebstahlversicherung.

Von ausländischen Versicherungsunternehmungen, die sich mit den zu Gruppe IV gehörigen Versicherungszweigen beschäftigten, unterstanden der Reichsaufsicht im Jahre 1910 insgesamt 42. Ein Teil davon hat nur ein ganz unbedeutendes deutsches Geschäft meist mit starker örtlicher Begrenzung, so daß es nicht angezeigt erschien, die Verhältnisse dieser Gesellschaften hier näher darzustellen. Daher enthält diese Statistik nur Angaben über 31 ausländische Versicherungsgesellschaften, nachdem gegenüber dem Vorjahre der Österreichische Phönix ausgeschieden ist. Von diesen Gesellschaften arbeiteten, soweit Versicherungszweige der Gruppe IV in Frage kommen, im Deutschen Reiche 17 nur in der Feuerversicherung, 9 (Nye danske Brandforsikrings-Selskab, Phoenix Assurance Company Limited in London, Yorkshire Insurance Company Limited in York, Die Niederlande, Niederländischer Lloyd, Assicurazioni Generali, Riunione Adriatica di Sicurtà, die Basler Versicherungs-Gesellschaft und die Helvetia, Schweizerische Feuerversicherungs-Gesellschaft in St. Gallen) in der Feuerund Einbruchdiebstahlversicherung und 5 (Erste Österreichische Allgegemeine UnfallVersicherungs-Gesellschaft, Schweizerische National-Versicherungs-Gesellschaft, Schweizerische Unfallversicherungs-Aktiengesellschaft, Union Suisse und die Zürich) allein in der Einbruchdiebstahlversicherung.

Bei den folgenden Ausführungen sowie bei der Betrachtung der Tabellen ist, soweit es sich um Feuerversicherung handelt, im Auge zu be- 
halten, daß es sich stets um die Gesamtheit der Versicherungen handelt; eine Trennung nach Risiken konnte nirgends vorgenommen werden; ebensowenig hat die Mobiliarfeuerversicherung von der Immobiliarfeuerversicherung geschieden werden können.

Uther den Umfang des Geschäfts der deutschen Unternehmungen in der Feuerversicherung Ende 1909 und Ende I 910 gibt Tabelle IV 1 Auskunft. Danach liefen an selbst abgeschlossenen Versicherungen einschließlich der in Rückdeckung gegebenen Teile:

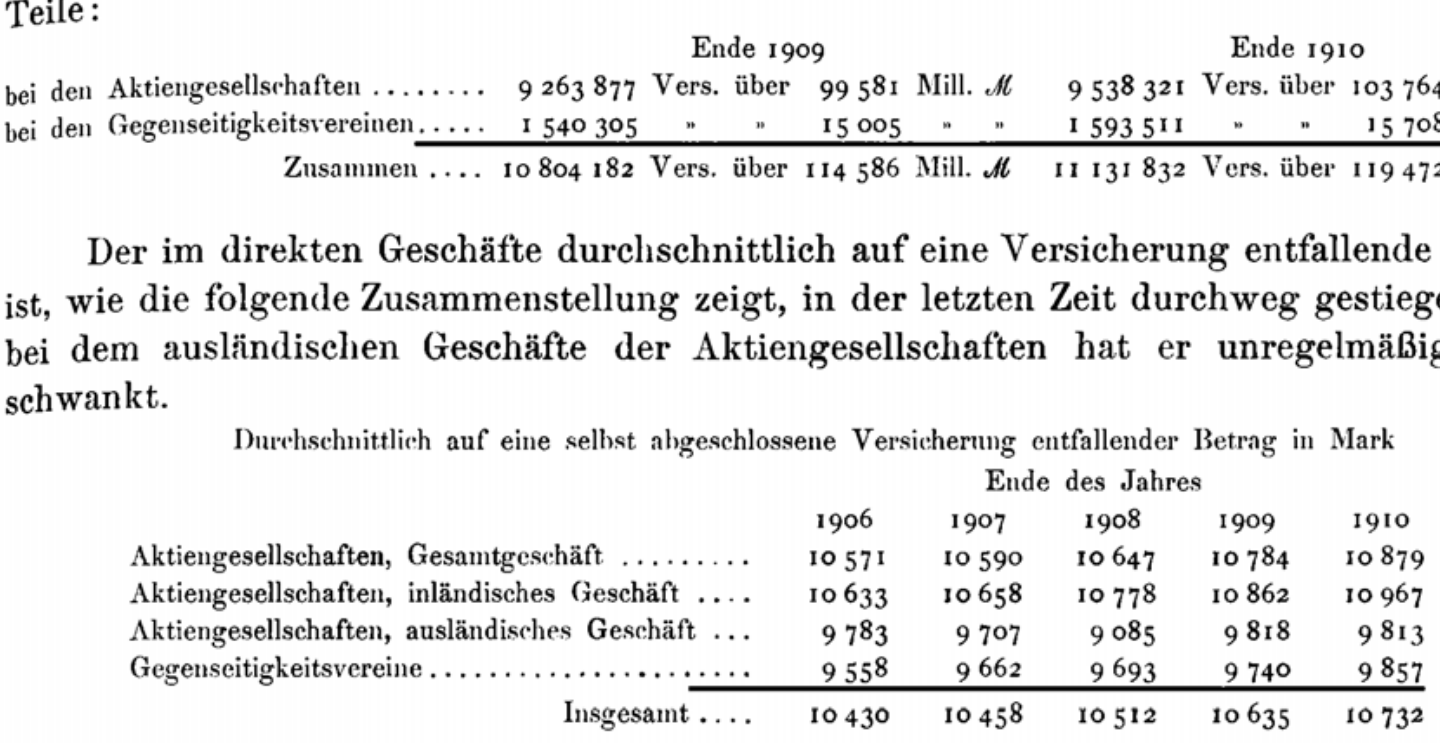

Die Aktiengesellschaften hatten außerdem noch Versicherungen in Höhe von 12975 Millionen Mark am Ende 1909 und in Höhe von 13554 Millionen Mark am Ende I 9 IO in Rückdeckung genommen, so daß die gesamte Bruttoversicherungssumme Ende I 909 112566 und Ende 1910 117318 Millionen Mark betrug. In dieser Summe sind alle diejenigen Beträge doppelt enthalten, welche von einer Gesellschaft, die in dieser Statistik behandelt wird, versichert und bei einer ebensolchen Gesellschaft in Rückdeckung gegeben sind. Dasselbe gilt von den unten angeführten Zahlen über die Gesamtversicherungssumme bei den anderen Versicherungszweigen. Der Anteil des mittelbaren Geschäfts am Gesamtgeschäfte beträgt 11,6 Prozent. Von den Gegenseitigkeitsvereinen haben zwei den geringen Betrag von zusammen 182 Millionen Mark in Rückdeckung übernommen.

Der für eigene Rechnung behaltene Teil stellte sich:

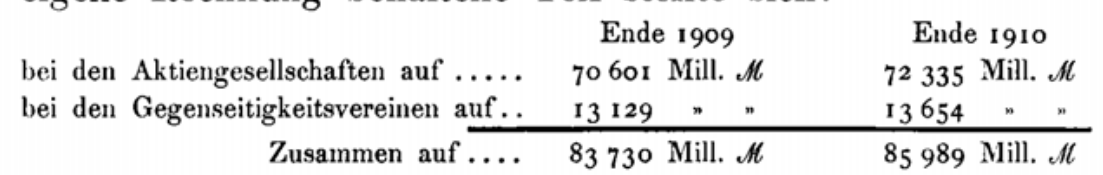

und betrug in Tausendsteln der überhaupt versicherten Summen:

$\begin{array}{lll}\text { bei den Aktiengesellschaften ...... } & 625 & 617 \\ \text { bei den Gegenseitigkeitsvereinen .. } & 866 & 858\end{array}$

Die Tabelle IV 2 behandelt lediglich das deutsche Geschäft der deutschen Aktiengesellschaften; aus dieser Tabelle in Verbindung mit Tabelle IV 1 ergibt sich folgende Teilung des Geschäfts:

Der Um. fang des Geschãfts der deutschen Unternehmungen 
Selbst abgeschlossene Versicherungen

inländisches Geschäft .............

ausländisches Geschäft ............

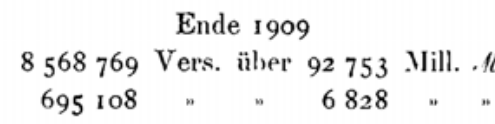

Von dem mittelbaren Geschäfte der Aktiengesellschaften kamen Ende I 909 auf das inländische Geschäft 1603 Millionen Mark und auf das ausländische Geschäft 11372 Millionen Mark, Ende I 910 auf das inländische Geschäft 1475 und auf das ausländische Geschäft 1207 a Millionen Mark.

Das gesamte inländische Geschäft der Aktiengesellschaften erstreckte sich Ende I 909 über 94356 Millionen Mark und Ende I9 го über 98034 Millionen Mark; das gesamte ausländische Geschäft bezifferte sich Ende I909 auf 18200 und Ende I9I0 auf 19284 Millionen Mark. Der Anteil der mittelbaren Versicherungen an der Gesamt. heit betrug danach beim inländischen Geschäft 1909 1,7 Prozent und I9 IO 1,5 Pro. zent, beim ausländischen dagegen I 909 62,5 Prozent und I 9 IO 62,6 Prozent.

Von den Gegenseitigkeitsvereinen haben nur drei (der Versicherungsverein Deut. scher Eisenbahnbediensteten, die Feuer- und Einbruchdiebstahl-Versicherungsanstalt des Verbandes Deutscher Beamtenvereine und die Gothaer Feuerversicherungsbank) ausländische Geschäfte, und zwar in geringem Maße, abgeschlossen. Alle zusammen hatten Ende I909 im Ausland 1682 Versicherungen über 51 Millionen Mark und Ende I9 Iо 1720 Versicherungen über denselben Betrag in Kraft.

In der Sturmschädenversicherung haben im Jahre ig Io dieselben beiden Aktiengesellschaften gearbeitet wie in den Vorjahren; der Erfolg ist auch in diesem Jahre kein großer gewesen. Nach Tabelle IV 3 liefen nämlich Ende 1909 insgesamt 1499 Policen über 204 Millionen Mark und Ende r9 10 1632 Policen mit 205 Millionen Mark. Von den Summen kamen Ende 1909 und Ende 1910 99,9 Prozent auf das deutsche Geschäft. Für eigene Rechnung wurde nur der geringe Betrag von 31 Millionen behalten. Rückversicherungen wurden von diesen beiden Gesellschaften nicht übernommen.

In der Wasserleitungsschädenversicherung haben Ende 1910 zwanzig deutsche Aktiengesellschaften gearbeitet, fünf mehr als im Vorjahre, nämlich die Cölnische Feuer-Versicherungs-Gesellschaft Colonia, die Rheinische FeuerversicherungsAktiengesellschaft Cöln, die Düsseldorfer Feuerversicherungs-Aktiengesellschaft in Düsseldorf, die Vaterländische Feuer-Versicherungs-Aktien-Gesellschaft in Elberfeld und der Deutsche Phönix, Versicherungs-Aktien-Gesellschaft in Frankfurt a. Main.

Nach Tabelle IV 3 liefen an selbst abgeschlossenen Versicherungen Ende 1909 101564 Policen über 4075 Millionen Mark und Ende r910 109768 Policen über 4430 Millionen Mark, was einen Zugang von 8204 Policen und 355 Millionen Mark Versicherungssumme bedeutet.

Die Gesellschaften hatten außerdem noch Versicherungen in Höhe von 82,1 Millionen Mark zu Ende 1909 und in Höhe von 54,7 Millionen Mark zu Ende 1910 in Rückdeckung genommen, so daß die gesamte Bruttoversicherungssumme Ende I910 4485 Millionen Mark betrug. Von den Versicherungssummen kamen Ende 1909 96,6 und Ende 1910 96,7 Prozent auf das deutsche Geschäft. Der weitaus größte Teil der Versicherungen, nämlich 3884 Millionen, wurde für eigene Rechnung behalten.

Sehr viel bedeutender ist das Geschäft in der Einbruchdiebstahlversicherung. Den Betrieb dieses Geschäftszweiges haben im Berichtsjahre sechs Aktien- 
gesellschaften aufgenommen, nämlich die Deutsche Reform-Versicherungsbank, Aktiengesellschaft in Berlin, die Preußische Feuer-Versicherungs-Aktien-Gesellschaft zu Berlin, die Rheinische Feuerversicherungs-Aktiengesellschaft in Cöln, dic Düsseldorfer Feuerversicherungs-Aktiengesellschaft in Düsseldorf, die Mitteldeutsche Versicherungs-Aktiengesellschaft in Halle a. S. und die Hansa, Allgemeine Versicherungs-Aktien-Gesellschaft in Hamburg.

Von den selbst abgeschlossenen Versicherungen der deutschen Aktiengesellschaften kamen:

$$
\begin{aligned}
& \text { Ende } 1909 \quad \text { Ende } 1910 \\
& \text { auf das inländische Geschäft...... } 75^{1} 507 \text { Vers. über } 10020 \text { Mill. } \mathscr{N} \quad 836029 \text { Vers. über II I } 2 \text { Mill. } \mathscr{M}
\end{aligned}
$$

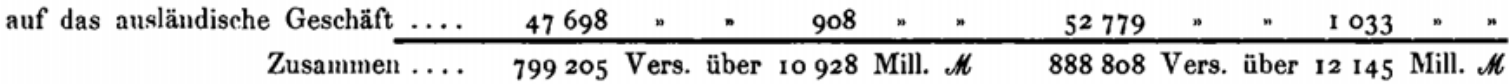

Das unmittelbare inländische Geschäft hat demnach um 84522 Versicherungen und 1092 Millionen Mark zugenommen, das ausländische um 5081 Versicherungen und 125 Millionen Mark. In Rückdeckung übernommen hatten diese Gesellschaften außerdem im inländischen Betrieb am Ende des Vorjahrs 1587 und am Ende des Berichtsjahrs 1645 Millionen Mark, so daß die gesamte Bruttoversicherungssumme des deutschen Geschäfts Ende I 90911607 Millionen Mark und Ende I910 12757 Millionen Mark betrug. Im ausländischen Geschäfte waren in Rückdeckung genommen Ende I 909 1267 und Ende 1910 1564 Millionen Mark; demnach ist die gesamte Bruttoversicherungssumme des ausländischen Geschäfts von 2175 auf 2597 Millionen Mark gestiegen. Der Anteil des indirekten Geschäfts am Gesamtgeschäft in der Einbruchdiebstahlversicherung belief sich danach beim inländischen Geschäft Ende 1909 auf 13,7 und Ende 1910 auf 12,9 Prozent, beim ausländischen Geschäft auf 58,3 bzw. 60,2 Prozent.

Das Geschäft der vier Gegenseitigkeitsvereine war auch im Jahre 1910 noch ganz gering, es stellte sich Ende des Jahres auf insgesamt 15533 Policen über 116 Millionen Mark. Es handelt sich lediglich um unmittelbare deutsche Versicherungen.

Alle 43 Unternehmungen zusammen hatten Ende I9 10 Versicherungen in Höhe von 15470 Millionen Mark in Kraft, davon liefen 10919 Millionen für eigene Rechnung.

Es ist bereits oben bemerkt, $\mathrm{da} ß$ von den ausländischen Versicherungsunternehmungen, welche im Deutschen Reiche die Feuerversicherung betreiben, in die vorliegende Statistik nur 26 aufgenommen sind. Hier soll nur ihr deutsches Geschäft kurz umschrieben werden.

Die 26 ausländischen Gesellschaften hatten im Deutschen Reiche selbst abgeschlossene Feuerversicherungen Ende 1909 in Höhe von 12762 Millionen Mark und Ende 1910 in Höhe von 13018 Millionen Mark; in Rückdeckung waren zu denselben Zeitpunkten Versicherungen im Betrage von 1821 und von 1763 Millionen Mark übernommen; der gesamte Versicherungsbestand betrug also in der Feuerversicherung am Schlusse des Vorjahrs 14583 und am Schlusse des Geschäftsjahrs 14780 Millionen Mark. Im Jahre I9 IO baben die selbst abgeschlossenen Versicherungen um 256; Millionen zugenommen und die in Rückdeckung übernommenen Versicherungen um 59 Millionen Mark abgenommen.

Der Anteil des indirekten Geschäfts am Gesamtgeschäft ist von 12,5 Prozent auf 11,9 Prozent zurückgegangen. 
Von den ausländischen Gesellschaften, die im Deutschen Reiche in der Einbruchdiebstahlversicherung gearbeitet haben, kommen hier vierzehn in Betracht; von diesen haben zehn lediglich unmittelbare, vier auch mittelbare Geschäfte betrieben. Die selbst abgeschlossenen Versicherungen beliefen sich Ende 1909 auf 611 Millionen Mark und Ende 1910 auf 688 Millionen Mark. Rechnet man die in Rückdeckung übernommenen Versicherungen hinzu, so erhält man für den Bestand des deutschen Geschäfts am Schlusse des Vorjahrs 688 und für den Bestand am Schlusse des Berichtsjahrs 784 Millionen Mark.

Der Umfang des gesamten direkten deutschen Feuer-

versicherungsgeschafts
Die Bruttopramien und Schaden a) der deutschen Gesellschaflen

Der Wert aller im Deutschen Reiche gegen Feuerschaden versicherten Gegenstände, wie er sich aus der Summe aller im Reiche unmittelbar versicherten Beträge ergibt, kann aus den vorstehenden Angaben allein nicht entnommen werden. Abgesehen von den verhältnismäßig kleinen Summen, die bei den kleinen Gegenseitigkeitsvereinen und bei den unter Landesaufsicht stehenden privaten Unternehmungen versichert sind, fehlen noch die Versicherungen von 51 öffentlichen Feuerversicherungs-Sozietäten. Uber den Umfang dieser Versicherungen veröffentlicht der Verband der öffentlichen Feuerversicherungs-Anstalten jährlich in den "Mitteilungen für die öffentlichen Feuerversicherungs-Anstalten “ Zusammenstellungen, deren Zuverlässigkeit nicht zu bezweifeln ist. Mit Hilfe dieser Angaben kann man den gesamten Feuerversicherungsbestand im Deutschen Reiche annähernd folgendermaßen feststellen:

Es waren an deutschen Werten versichert in Millionen Mark:

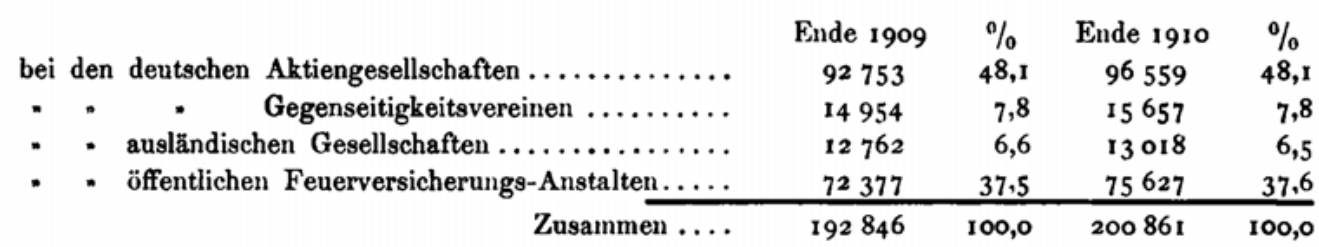

Die Bruttoprämieneinnahme im Berichtsjahr und die im Berichtsjahr entstandenen Bruttoschäden aller Arten von Risiken, jedoch ohne Schadenermittelungskosten, sind für die deutschen privaten Unternehmungen in den Tabellen IV 8 bis 10 und für das deutsche Geschäft der ausländischen Gesellschaften in den Tabellen IV 11 und 12 zusammengestellt.

In der Feuerversicherung haben danach im Jahre 19 Io 36 deutsche Aktiengesellschaften $224151446 \mathscr{N}$ und 20 Gegenseitigkeitsvereine $40582832 \mathscr{M}$, alle diese Unternehmungen zusammen also $264734278 \mathscr{M}$ an Prämien eingenommen; in diesem Jahre sind Schäden (einschließlich des Anteils der Rückversicherer, aber ohne Schadenermittelungskosten) bei den Aktiengesellschaften im Betrage von $122628510 \mathscr{M}$, bei den Gegenseitigkeitsvereinen im Betrage von $13031393 \mathcal{M}$, bei allen Anstalten zusammen im Betrage von $135659903 \mathscr{M}$ entstanden. Dabei ist zu beachten, daß einige Gegenseitigkeitsvereine, um Nachschüsse zu vermeiden, Vorprämien erheben, die den regelmäßigen Bedarf weit übersteigen und den daraus entstehenden Uberschuß den Versicherten wieder zuführen (vgl. Tabelle IV 21).

Der durchschnittliche Betrag eines Schadens aus selbst abgeschlossenen Feuerversicherungen (ohne Ermittelungskosten) stellte sich bei den Aktiengesellschaften im inländischen Geschäft ouf $358 \mathscr{N}$, im ausländischen Geschäft auf $980 \mathscr{M}$ gegen $334 \mathscr{M}$ bezw. $1123 \mu$ im Vorjahre. 
Bei den Gegenseitigkeitsvereinen hat der Schadenfall aus selbst abgeschlossenen Versicherungen im inländischen Geschäfte durchschnittlich $422 \mathscr{N}$, im ausländischen (37 Fälle) Geschäfte durchschnittlich $544 \mathscr{M}$ erfordert.

Die beiden Gesellschaften, die in der Versicherung gegen Sturmschäden gearbeitet haben, erzielten ('Tabelle IV 9) aus diesem Geschäft eine Prämieneinnahme von $168712 \mathscr{N}$, welche nur aus dem deutschen Betriebe herrührte. An Schäden sind aus selbst abgeschlossenen Versicherungen 86 mit $29 \check{0} 03 \mathscr{M}$ (ohne Ermittelungskosten) zu verzeichnen gewesen; der durchschniltliche Betrag eines Schadens stellte sich demnach auf $343 \mathscr{M}$.

Die zwanzig Gesellschaften, die in der Versicherung gegen Wasserleitungsschäden gearbeitet haben, erzielten aus diesem Geschäfte ('Tabelle IV 9) eine Prämieneinnahme von $2548808 \%$. An Schäden sind 739034 . M (ohne Ermittelungskosten) zu verzeichnen gewesen. Der durchschnittliche Betrag eines Schadens aus selbst abgeschlossenen Versicherungen stellte sich beim inländischen Geschäft auf $44 \mathscr{M}$, beim ausländischen Geschäft auf $62 \mathscr{N}$.

In der Einbruchdiebstahlversicherung (Tabelle IV 10) haben die deutschen Aktiengesellschaften $13493410 \mathscr{N}$ an Prämien eingenommen und $4849469 \mathscr{M}$ für Schäden zu verausgaben gehabt; die vier andern Unternehmungen haben $76552 \mathscr{M}$ Prämien eingezogen und 18248 für Schäden gezahlt.

Der durchschnittliche Betrag eines Schadens aus den selbst abgeschlossenen Versicherungen stellte sich bei den Aktiengesellschaften (ohne Ermittelungskosten) im inländischen Geschäft auf $163 \mathscr{M}$ und im ausländischen Geschäft auf $2123 \mathscr{N}$, bei den Gegenseitigkeitsvereinen auf $97 \mathscr{M}$.

Für das deutsche Feuerversicherungsgeschäft der 26 ausländischen Gesellschaften gelten folgende Zahlen ('Tabelle IV 11). Die Prämieneinnahme belief sich auf $28054113 \mathscr{M}$; für Schäden waren $13824233 \mathscr{M}$ aufzuwenden. Von den Prämien stammten 85,1 Prozent aus selbst abgeschlossenen Versicherungen und 14,9 Prozent aus Rückversicherungen, von den Schäden betrafen 82,2 Prozent selbst abgeschlossene Versicherungen und 17,8 Prozent in Rückdeckung übernommene Versicherungen.

Das deutsche Einbruchdiebstahlversicherungsgeschäft der hier in Betracht kommenden 14 ausländischen Gesellschaften, das im wesentlichen unmittelbar betrieben wird, zeigt sich auch hinsichtlich der Prämieneinnahme und der Schadenleistungen (Tabelle IV 12) als wenig bedeutend. Die Prämieneinnahme hat nur $885676 \mathscr{M}$ betragen, für Schäden sind $357128 \mathscr{M}$ zu zahlen gewesen.

Wie oben die Versicherungssummen annähernd für das gesamte direkte deutsche Feuerversicherungsgeschäft ermittelt wurden, so können mit Hilfe der vorstehenden Zahlen und der Angaben in den "Mitteilungen für die öffentlichen FeuerversicherungsAnstalten " auch die Schadenbeträge, die den deutschen Geschädigten zugeflossen sind, angenähert festgestellt werden.

\begin{tabular}{|c|c|c|c|c|}
\hline \multirow[t]{3}{*}{ 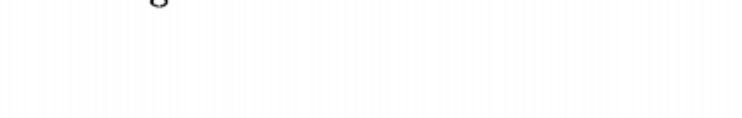 } & \multicolumn{4}{|c|}{ Schadenzahlungen } \\
\hline & \multicolumn{2}{|c|}{ im Jahre 1909} & \multicolumn{2}{|c|}{ im Jahre rgro } \\
\hline & $1000 \mathscr{N}$ & $\%$ & $1000 \mathscr{N}$ & $\%$ \\
\hline deutsche Aktiengesellschaften $\ldots \ldots \ldots \ldots \ldots \ldots \ldots$ & 73686 & 45,7 & 76707 & 46,8 \\
\hline Gegenseitigkeitsvereine............... & 12653 & 7,8 & 12718 & 7,8 \\
\hline ausländische Gesellschaften $\ldots \ldots \ldots \ldots \ldots \ldots \ldots \ldots$ & 12889 & 8,0 & $1135^{8}$ & 6,9 \\
\hline deutsche öffentliche Feuerversicherungs-Anstalten ..... & 62105 & 38,5 & 62985 & 38,5 \\
\hline Zusammen .... & 161333 & 100,0 & 163768 & 100,0 \\
\hline
\end{tabular}

b) der auslandischen Gesellschaften eutschen Feuergeschsfte 
Das ausländische Geschsft der deutschen und das deutsche Geschaft der ausIsndisehe Gesellachaften

Fe

Feuerversicherung $\ldots \ldots \ldots \ldots \ldots \ldots \ldots \ldots \ldots \ldots$

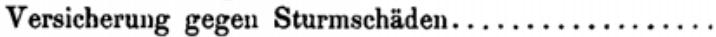

- Wasserleitungsschäden ..........

" Einbruchdiebstahl .............

Zusammen ....

Eine Vergleichung der Bruttoprämien und Schäden aus dem ausländischen ('ieschäfte der deutschen Gesellschaften mit den entsprechenden Beträgen aus dem deutschen Geschäfte der ausländischen Gesellschaften führt zu folgender Zusammenstellung:

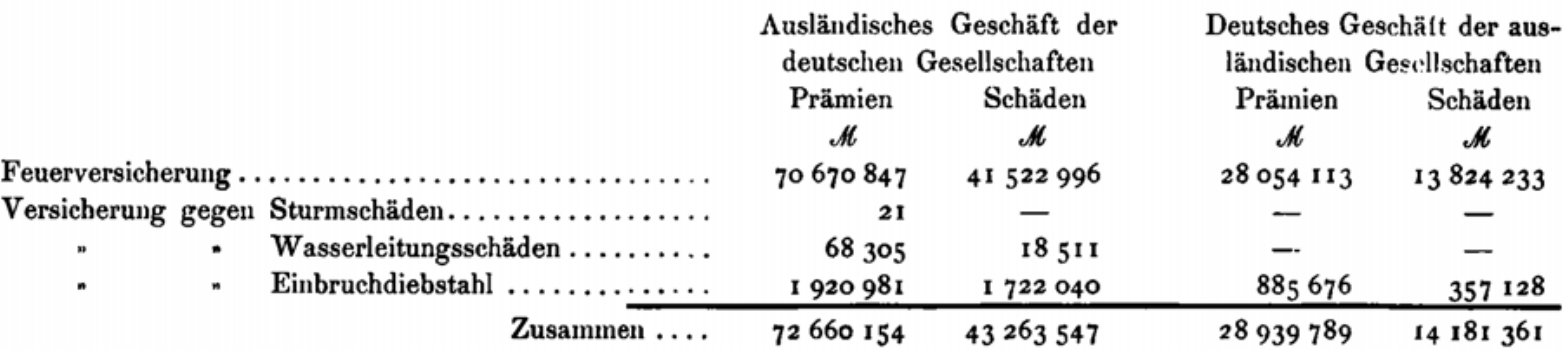

Es übertrifft also das ausländische Geschäft der deutschen Unternehmungen das deutsche Geschäft der im Inland auf diesem Gebiet arbeitenden ausländischen Gesellschaften ganz bedeutend. Das kann aber auch nicht überraschen, da sich die deutsche Versicherung in fast allen wichtigeren Staaten der Welt zu betätigen weiß. Es arbeiteten, um nur diejenigen Länder zu nennen, in denen deutsche Gesellschaften besonders zahlreich vertreten sind, in der Feuerversicherung im Jahre 1910 in clen Niederlanden 18, in Belgien 17, in der Schweiz 16, in Österreich 14, in Dänemark 13, in England und in Schweden je 11, in Rußland 10, in Frankreich und in Luxemburg je 9, in Italien und Norwegen je 6 Gesellschaften usw. Die Nebenzweige der Feuerversicherung haben naturgemäß erst eine weniger weite Verbreitung gefunden; doch arbeiteten in der Einbruchdiebstahlversicherung im Jahre 19 ro in den Niederlanden bereits 18 , in der Schweiz 15, in Belgien 11, in Dänemark 10, in Österreich 8, in Frankreich und Schweden je 7 Gesellschaften, in Norwegen 6 usw.; die Versicherung gegen Wasserleitungsschäden haben in Dänemark 6, in der Schweiz 5, in len Niederlanden 4, in Österreich und Schweden je 3 deutsche Gesellschaften, in Belgien und Ungarn je eine deutsche Gesellschaft betrieben.

Die Feuerversicherungs-Aktiengesellschaften arbeiten außer auf dem Gebiete der Feuerversicherung meistens auch noch in anderen Versicherungszweigen, ohne daß sie in ihren Büchern die sämtlichen Einnahme- und Ausgabeposten streng nach Geschäftszweigen trennten. Es ist jedoch seit einer Reihe von Jahren angängig, die Verwaltungskosten vollständig auf die einzelnen Zweige aufzuteilen, so daß die Betriebs-Einnahmen den Betriebs-Ausgaben nebst Verwaltungskosten und Steuern gegenübergestellt und somit der aus dem Versicherungsgeschäft allein herrührende Betriebsgewinn des Berichtsjahrs hergeleitet werden kann ('Tabelle IV 13 bis 15). Der Gewinn aus dem Gesamtgeschäfte wird in der Bilanz (Tabelle IV 19) nachgewiesen.

Der Gleichförmigkeit wegen sind die Aktiengesellschaften und die Gegenseitigkeitsvereine, die nur einen Geschäftszweig pflegen, ebenso behandelt worden.

Bei Gruppe IV sind als Betriebs-Einnahmen die Prämien des Geschäftsjahrs für eigene Rechnung (Jahreseinnahme nach Abzug der Ristorni und der verausgabten Rückversicherungsprämien), die Nebenleistungen der Versicherten, die Ersparnis aus der Schadenreserve des Vorjabrs und die Abnahme der Prämienüberträge zusammengefaßt; als Betriebs-Ausgaben kommen die Zahlungen und Rückstellungen für Schäden (einschließlich Schadenermittelungskosten), die im Geschäftsjahr entstanden sind (nach Ab- 
„ug der Leistungen der Rückversicherer, aber einschließlich der Schadenermittelungskosten), die Leistungen für gemeinnützige Zwecke, der Zuschuß zur Schadenreserve des Vorjahrs und die Zunahme der Prämienüberträge in Betracht.

Bei den deutschen Unternehmungen betrugen in der Feuerversicherung:

\begin{tabular}{|c|c|c|c|c|}
\hline & & $\begin{array}{l}\text { bei der Gesamtheit } \\
\text { der Unternehmungen }\end{array}$ & $\begin{array}{l}\text { bei } 36 \text { Aktien- } \\
\text { gesellschaften }\end{array}$ & $\begin{array}{l}\text { bei } 20 \text { Gegen- } \\
\text { seitigkeitsvereinen }\end{array}$ \\
\hline die Summe & der Betriebs-Eimnahmen .... & $166864737 \mathscr{H}$ & $129218446 \mathscr{M}$ & $37646291 \mathscr{M}$ \\
\hline , & " Betriebs- $\Lambda$ usgaben ..... & $8477955^{2}$. & 72833280 . & I I 946272 " \\
\hline
\end{tabular}

Von je $1000 \varkappa$ der Betriebs-Einnahmen der Feuerversicherung kamen:

\begin{tabular}{|c|c|c|c|}
\hline & $\begin{array}{l}\text { bei der Gesamtheit } \\
\text { der Unternehmungen }\end{array}$ & $\begin{array}{l}\text { bei den } 36 \text { Aktiẹn- } \\
\text { gesellschaften }\end{array}$ & $\begin{array}{l}\text { bei den } 20 \text { Gegen- } \\
\text { seitigkeitsvereincn }\end{array}$ \\
\hline auf Nettoprämien.................. & $956 \mathscr{A C}$ & $950 \approx$ & $978 \mathscr{H}$ \\
\hline " Nebenleistungen der Versicherten ... & $12 "$ & 12 " & $9 *$ \\
\hline " Ersparnis aus der Schadenreserve... & $3 I^{\prime}$ & $3^{8} n$ & $7 \%$ \\
\hline " $\Lambda$ bnahme der Prämicnüberträge..... & $\mathrm{I}$ " & . & $6 \cdot$ \\
\hline & у $000 \mathscr{A}$ & I $000 A$ & 1 $000 \mathscr{H}$ \\
\hline
\end{tabular}

Von je $1000 \mathscr{N}$ der Betriebs-Ausgaben kamen:

\begin{tabular}{|c|c|c|c|}
\hline & $\begin{array}{l}\text { bei der Gesamtheit } \\
\text { der Unternchmungen }\end{array}$ & $\begin{array}{l}\text { bei den } 36 \text { Aktien- } \\
\text { gesellschaften }\end{array}$ & $\begin{array}{l}\text { bei den } 20 \text { Gegen- } \\
\text { seitigkeits:ereinen }\end{array}$ \\
\hline auf gezahlte Schäden $\ldots \ldots \ldots \ldots \ldots \ldots$ & $693 \pi$ & $679 \mathscr{A}$ & $778 \mathscr{A}$ \\
\hline " zurückgestellte Schäden.......... & $197 \cdots$ & $211 \cdots$ & $116 ”$ \\
\hline - Leistungen für gem. Zwecke ...... & 28 & $29 "$ & 38 \\
\hline " Zuschuß zur Schadenreserve $\ldots . .$. & . & . & . \\
\hline \multirow{2}{*}{ " Zunahme der Prämienüberträge .... } & $82 n$ & $81 \cdot$ & $88 \cdots$ \\
\hline & I $000 \mathscr{M}$ & I $000 \Omega$ & $1000 \mathscr{M}$ \\
\hline
\end{tabular}

Von den im Geschäftsjahr entstandenen Schadensummen wurden durchschnittlich bei den Aktiengesellschaften 76,3 Prozent, bei den Gegenseitigkeitsvereinen 87,0 Prozent und bei allen Anstalten zusammen 77,8 Prozent gezahlt. Der Rest wurde der Schadenreserve überwiesen. -- Von den Leistungen für gemeinnützige Zwecke beruhten bei den Aktiengesellschaften 64,5 Prozent und bei den Gegenseitigkeitsvereinen 84,1 Prozent auf gesetzlicher Vorschrift.

An Verwaltungskosten und Steuern hatten für die Feuerversicherung die 36 Aktiengesellschaften $42177965 \mathscr{N}$ und die 20 Gegenseitigkeitsvereine $5836137 \mathscr{M}$ aufgewandt.

Einen Verlust aus dem Betriebe der Feuerversicherung des Berichtsjahrs von zusammen $746582 \mathscr{M}$ haben fünf Aktiengesellschaften nachgewiesen; den übrigen Gesellschaften hat das Berichtsjahr einen Gewinn von insgesamt $14953783 \mathscr{N}$ gebracht.

Von den Gegenseitigkeitsvereinen hatte einer einen Verlust, und zwar von $37085 \mathscr{N}$; die übrigen hatten einen Gewinn von insgesamt $19900967 \mathscr{N}$. Zu dieser letzten Zahl ist jedoch zu bemerken, daß allein $15983566 \mathscr{M}$ auf die Gothaer Feuerversicherungsbank und 2674626 $\mathscr{N}$ auf die Württembergische Privatfeuerversicherung kommen. Diese Überschüsse sind kein Gewinn im gewöhnlichen Sinne; sie haben vielmehr in der Art der Prämienbemessung ihren Grund, da die genannten Unternehmungen Prämien erheben, die den voraussichtlichen Bedarf weit übersteigen; der Uberschuß wird den Versicherten dann im folgenden Jahre zurückerstattet (vgl. S. 60 und "Entwickelung “S. 39). 
Zu der Sturmschäden-, der Wasserleitungsschäden- und der Einbruch diebstahlversicherung ist hier folgendes $\mathrm{zu}$ bemerken:

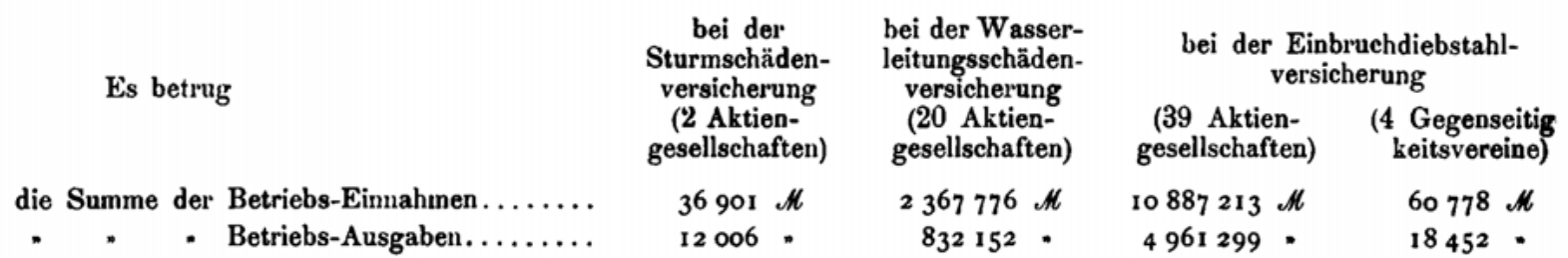

Von je $1000 \mathscr{M}$ der Betriebs-Einnahmen kamen:

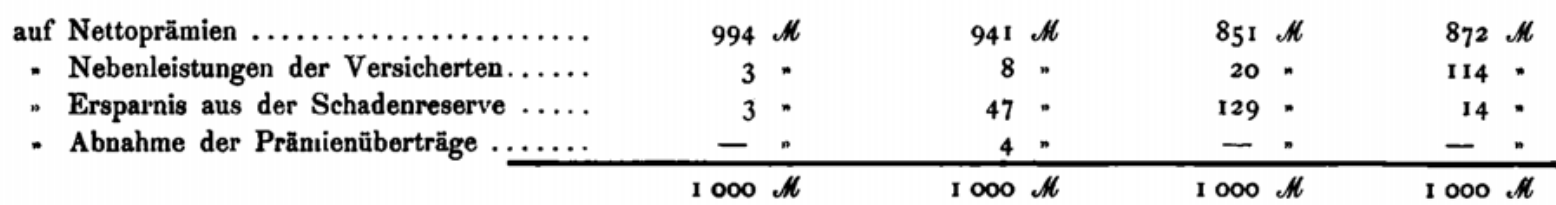

Von je $1000 \mathscr{M}$ der Betriebs-Ausgaben kamen:

\begin{tabular}{|c|c|c|c|c|c|}
\hline auf gezahlte Schäden & & $\mathscr{N}$ & $569 \mathscr{A}$ & $393 \mathscr{H}$ & $578 \cdot \mathscr{H}$ \\
\hline - zurückgestellte Schäden $\ldots \ldots \ldots \ldots \ldots$ & 35 & • & $279 \cdot$ & $402 n$ & $88 \cdots$ \\
\hline " Zuschuß zur Schadenreserve .......... & - & $"$ & . & $3 \cdot$ & - \\
\hline - Zunahme der Prämienũberträge..... . & 215 & $n$ & 152. & $202 n$ & $334 "$ \\
\hline
\end{tabular}

Von den im Geschäftsjahr entstandenen Nettoschäden wurden bei der Sturmschäden versicherung 95,5 Prozent, bei der Wasserleitungsschädenversicherung 67,1 Prozent unı bei der Einbruchdiebstahlversicherung 49,5 Prozent noch im Geschäftsjahre beglichen der Rest wurde in Schadenreserve gestellt.

An Verwaltungskosten und Steuern hat die Sturmschädenversicherung $12024 \mathscr{N}$ die Wasserleitungsschädenversicherung $950771 \mathscr{M}$ und die Versicherung gegen Einbruch diebstahl bei den Aktiengesellschaften $3739883 \mathscr{M}$ und bei den Gegenseitigkeitsvereine] $15244 \mathscr{N}$ erfordert. Die Sturmschädenversicherung hat einen Gewinn von $12871 \mathcal{A}$ gebracht. In der Wasserleitungsschädenversicherung haben 6 Gesellschaften einen Ver lust von zusammen $29760 \mathscr{M}$ und 14 einen Gewinn von zusammen $614613 \mathscr{M}$ gehabt In der Einbruchdiebstahlversicherung ist bei 6 Aktiengesellschaften ein Verlust von zu sammen $40632 \mathscr{M}$, bei den übrigen 33 ein Gewinn von zusammen $2226663 \mathscr{M}$ zu ver zeichnen. Das kleine Geschäft der vier Gegenseitigkeitsvereine hat einen Verlust vol $2285 \mathscr{M}$ und einen Gewinn von $29367 \mathscr{M}$ gebracht.

Das Ergebnis der Vermögensverwaltung wird von den Unternehmungen in der Rege nicht nach den einzelnen Geschäftszweigen getrennt; nur der Lebensversicherung werdes immer bestimmte Erträge überwiesen, weil das der Betrieb dieses Geschäfts erfordert Es besteht keine Veranlassung, in dieser Statistik von dem Verfahren der Gesellschaftes abzugehen. Soweit also nicht ein Teil des Ertrags einem bestimmten Zweige zuge wiesen ist, wird das Ergebnis nur insgesamt aufgeführt und im allgemeinen bei der jenigen Gruppe mitgeteilt, zu der das Hauptgeschäft der Unternehmung gehört.

In Tabelle IV 16 findet sich bei 36 Aktiengesellschaften der Frtrag der gesamte Vermögensverwaltung und bei 2 Gesellschaften nur der nicht der Lebensversicherun, zukommende Teil; der gesamte Ertrag ist für eine Gesellschaft bei Gruppe I, für ein 
andere Anstalt bei Gruppe II nachgewiesen. Bei den Feuerversicherungs-Gegenseitigkeitsvereinen ist der Ertrag der gesamten Vermögensverwaltung in Tabelle IV 16 aufgeführt.

Unter den Erträgen stehen überall die Zinsen im Vordergrund mit $9307265 \mathscr{M}$ bei den Aktiengesellschaften und $2776582 \mathscr{M}$ bei den Gegenseitigkeitsvereinen. Der reine Ertrag der gesamten Vermögensverwaltung beläuft sich auf $9857942 \mathscr{N}$ und $2865791 \mathscr{M}$.

Eine Bilanz ist in dieser Statistik grundsätzlich nur für das Gesamtgeschäft einer Die Bilanz jeden Gesellschaft gegeben und bei der Gruppe aufgeführt, zu der das Unternehmen seinem Hauptberufe nach - die Transportversicherung ausgeschlossen - gehört. Dementsprechend sind die Bilanzen der Providentia und der Bayerischen VersicherungsBank bei Gruppe I und der Allianz bei Gruppe II behandelt, so daß hier nur die Bilanzen für 35 deutsche Aktiengesellschaften und 20 Gegenseitigkeitsvereine darzustellen sind. Faßt man diese zusammen, so schließt die Bilanz für die Aktiengesellschaften mit $545669543 \mathscr{M}$ und für die Gegenseitigkeitsvereine mit $80176952 \mathscr{M}$ ab. Im übrigen mögen folgende Zahlen hier zusammengestellt werden:

Aktiva

Verpflichtungen der Aktionäre oder Garanten.....

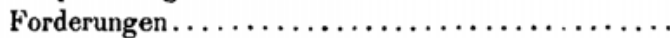

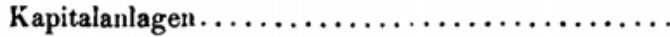

Kassenbestand $\ldots \ldots \ldots \ldots \ldots \ldots \ldots \ldots \ldots \ldots \ldots$

Passiva

Aktien- oder Garantiekapitai $\ldots \ldots \ldots \ldots \ldots \ldots$

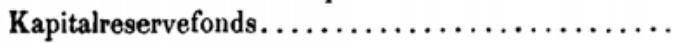

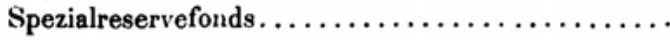

Guthaben anderer Versicherungsunternehmungen...

Prämienüberträge $\ldots \ldots \ldots \ldots \ldots \ldots \ldots \ldots \ldots \ldots$

Schadenreserve $\ldots \ldots \ldots \ldots \ldots \ldots \ldots \ldots \ldots \ldots \ldots$

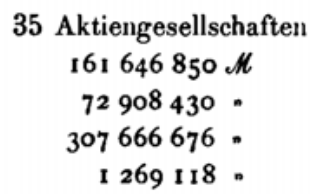

35 Aktiengesellschaften I61 $646850 \mathscr{M}$ 72908430 . 307666676 . 1269118 .

20 Gegenseitigkeitsvereine $946000 \mathcal{N}$ 7138958 . 70587195 . $279665 \cdot$

Die Kapitalanlagen (Tabelle IV 20) verteilen sich auf folgende Wertarten:

\begin{tabular}{|c|c|c|c|c|c|c|}
\hline \multirow[b]{2}{*}{ Grundbesitz $\ldots \ldots \ldots \ldots \ldots \ldots \ldots \ldots \ldots \ldots \ldots \ldots$} & \multicolumn{2}{|c|}{ bei 35 Aktiengesellschaften } & \multicolumn{4}{|c|}{ bei 20 Gegenseitigkeitsvereinen } \\
\hline & 2899227 I $\mathscr{M}$ oder & $9,4 \%$ & 3346374 & $\mathscr{N}$ oder & & $\%$ \\
\hline Hypotheken und Grundschulden ............. & $165434204=$ & $53,8 \cdot$ & 32701622 & n & 46,3 & n \\
\hline Darlehen an öffentliche Körperschaften......... & $198000 \cdot$ & $0,1 \cdots$ & 7857497 & $n$ & $\mathbf{I} \mathbf{1}, \mathbf{I}$ & $\cdot$ \\
\hline 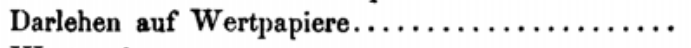 & I 340625 " & $0,4 \cdot$ & 100000 & * & 0,2 & * \\
\hline 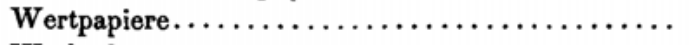 & 100449861 & 32,6 . & 25463796 & n & 36,1 & * \\
\hline Wechsel $\ldots \ldots \ldots \ldots \ldots \ldots \ldots \ldots \ldots \ldots \ldots \ldots \ldots$ & $3703673 \cdot$ & $1,2 \cdots$ & 80140 & n & $\mathbf{0 , \mathbf { I }}$ & " \\
\hline Sonstige Anlagen $\ldots \ldots \ldots \ldots \ldots \ldots \ldots \ldots \ldots$ & $7548042=$ & $2,5 n$ & 1037766 & n & 1,5 & $\cdot$ \\
\hline
\end{tabular}

Vergleicht man die Kapitalanlagen der in der Bilanz aufgeführten Unternehmungen am Ende des Berichtsjahrs mit den entsprechenden Anlagen am Ende des Vorjahrs, so findet man eine Zunahme um $233039 \mathscr{M}$ bei dem Grundbesitz, um $15752987 \mathscr{M}$ bei den Hypotheken und Grundschulden, um $1333714 \mathscr{N}$ bei den Darlehen an öffentliche Körperschaften, um $720875 \mathscr{M}$ bei den Darlehen auf Wertpapiere, um $8559638 \mathscr{M}$ bei dem Buchwerte der Wertpapiere (um rund 10 Millionen bei dem Nennwert) und um $2323300 \mathscr{M}$ bei den sonstigen Anlagen. Zurückgegangen ist der Bestand an Wechseln um $18195 \mathscr{M}$.

Die Bilanz weist bei zwei Gegenseitigkeitsvereinen einen Verlust von zusammen $30252 \mathcal{M}$ nach. Alle anderen Unternehmungen haben günstig abgeschlossen. Der bilanz- 
mäßige Gewinn beträgt für die Aktiengesellschaften $27242051 \mathscr{M}$ und für die Gegen seitigkeitsvereine $2243432 \vec{\imath} \mathscr{M}$; er ist nach Tabelle IV 21 wie folgt verteilt worden

\begin{tabular}{|c|c|c|c|c|c|c|}
\hline \multirow[b]{2}{*}{ an den Kapitalreservefonds........... } & \multicolumn{3}{|c|}{35 Aktiengesellschaften } & \multicolumn{3}{|c|}{20 Gegenseitigkeitsvereine } \\
\hline & I 048208 & $\mathscr{M}$ oder & $3,8 \%$ & 881575 & $\mathscr{N}$ oder & $\therefore 9 \%$ \\
\hline an sonstige Reserven $\ldots \ldots \ldots \ldots \ldots \ldots$ & 4831791 & $\bullet \quad "$ & 17,7 & 561703 & " & 2,5 . \\
\hline an den Beamtenfonds. & 696301 & $"$ & 2,6 . & 255693 & $n$ & $1,2 \cdot$ \\
\hline an die Aktionäre (Garanten)..$\ldots \ldots \ldots$ & 14194399 & $n$ & $52,1 \cdots$ & - & $n$ & $-\cdot$ \\
\hline für Tantiemen...$\ldots \ldots \ldots \ldots \ldots \ldots \ldots$ & 2290393 & $n$ & $8,4 \cdots$ & 6242 & $n$ & . $\cdot$ \\
\hline die Versicherten..$\ldots \ldots \ldots \ldots \ldots$ & - & $n$ & $-\cdots$ & 20468426 & $n$ & $9:, 2$ ? \\
\hline$\ldots \ldots \ldots \ldots$ & I 846297 & $n$ & $6,8 \cdots$ & 43500 & $n$ & 0,2 , \\
\hline Tortrag auf neue Rechnung $\ldots \ldots \ldots \ldots$ & 2334662 & $n$ & $8,6 n$ & 217188 & $n$ & 1,0 \\
\hline Zusamme & 27242051 & de & $0,0 \%$ & 434327 & $\mathscr{M}$ oder & $x, 0^{0 \prime}$ \\
\hline
\end{tabular}

Für die ausländischen Gesellschaften sind die Bilanz der Assicurazioni General bereits bei der Lebensversicherung in Tabelle I 47 und die Bilanzen der Ersten Öster reichischen Allgemeinen Unfall-Versicherungs-Gesellschaft, der Schweizerischen National Versicherungs-Gesellschaft, der Schweizerischen Unfallversicherungs-Gesellschaft und des Zürich bei der Unfall- und Haftpflichtversicherung in Tabelle II 14 gegeben; die Bi; lanzen der übrigen 25 Unternehmungen finden sich in Tabelle IV 23. Im Anschlussi daran sind in Tabelle IV 24 die Kapitalanlagen und die Aktivforderungen erläutert Wegen des großen Interesses, das der Art der Kapitalanlagen zukommt, sind in dei folgenden Ubersicht die Gesellschaften nach Heimatländern zusammengefaßt; zum Ver gleiche sind die Verhältniszahlen für die deutschen Unternehmungen wiederholt.

Von je $1000 \mathscr{M}$ Kapitalanlagen entfielen auf:

\begin{tabular}{|c|c|c|c|c|c|c|c|c|}
\hline bei & $\begin{array}{l}\text { Grund- } \\
\text { besitz }\end{array}$ & $\begin{array}{l}\text { Hypo- } \\
\text { theken } \\
\text { und } \\
\text { Grund- } \\
\text { schulden }\end{array}$ & $\begin{array}{l}\text { Darlehen } \\
\text { an öffent- } \\
\text { liche } \\
\text { Körpcr- } \\
\text { schaften }\end{array}$ & $\begin{array}{c}\text { Darlehen } \\
\text { auf } \\
\text { Wert- } \\
\text { papiere }\end{array}$ & $\begin{array}{l}\text { Wert- } \\
\text { papiere }\end{array}$ & Wechsel & $\begin{array}{l}\text { Sonstige } \\
\text { Anlagen }\end{array}$ & $\begin{array}{c}\text { Gesamtbetrag } \\
\text { der } \\
\text { Kapitalanlagen }\end{array}$ \\
\hline 2 belgischen Gesellschaften ........ & 34 & - & - & 27 & 939 & - & - & IV $134865 d$ \\
\hline 1 dänischen Gesellschaft .......... & 169 & 567 & - & - & 264 & - & - & $: 321416$ \\
\hline 14 englischen Gesellschaften ....... & 121 & 125 & - & 19 & 681 & 3 & 51 & 183,872870 . \\
\hline 1 französischen Gesellschaft ....... & 123 & - & - & - & 877 & . & - & 2i $476909 \cdot$ \\
\hline 2 niederländischen Gesellschaften... & 156 & 117 & - & 179 & 542 & - & 6 & I 492433 n \\
\hline 1 österreichischen Gesellschaft ...... & 95 & - & - & $\mathbf{I}$ & 871 & 33 & - & I. 282849 . \\
\hline 2 schwedischen Gesellschaften ..... & 57 & 542 & - & 48 & 224 & - & I 29 & 7:018805 \\
\hline $\begin{array}{l}2 \text { schweizerischen Gesellschaften.... } \\
\text { dagegen bei }\end{array}$ & 96 & 392 & - & - & 457 & 55 & - & 1: 317738 n \\
\hline deutschen Aktiengesellsch & 94 & $53^{8}$ & I & 4 & 326 & 12 & 25 & $30^{\prime} 666676$, \\
\hline 20 deutschen Gegenseitigkeitsvereinen & 47 & 463 & 111 & 2 & $36 r$ & $\mathbf{I}$ & 15 & $7 \cdot 587195 \cdots$ \\
\hline
\end{tabular}

Die kleinen Unternehmungen
Von den 12 kleinen Vereinen, die in den Tabellen nicht behandelt sird, muf einer wegen unvollständiger Angaben hier ganz ausscheiden, bei den übrigen kommer insgesamt 50273 Versicherungen mit rund 446,09 Millionen Mark in Frage; de durch. schnittliche Betrag einer Versicherung stellt sich danach auf $8873 \mathscr{M}$. Die gesamter Einnahmen haben im Jahre ig ro $457171 \mathscr{M}$ betragen; davon kommen $280451 \mathscr{M}$ odes 61,3 Prozent auf Prämien, $14071 \mathscr{M}$ oder 3,1 Prozent auf Nebenleistungen ler Ver. sicherten und $23229 \mathscr{M}$ oder 5,1 Prozent auf Kapitalerträge; von den gesamen Aus. gaben in Höhe von $402134 \mathscr{N}$ kommen $297943 \mathscr{N}$ oder 74,1 Prozent auf Entschä 
digungen und $54340 \mathscr{M}$ oder 13,5 Prozent auf Verwaltungskosten. Als Vermögen am Schlusse des Berichtsjahrs haben die elf Vereine zusammen $889269 \mathscr{M}$ nachgewiesen.

Diese Zahlen lassen jedoch kein Urteil über die Bedeutung der kleinen Vereine im Deutschen Reiche zu. Die Zahl der überhaupt vorhandenen kleinen Vereine ist viel größer; auch werden sich unter der Aufsicht der Landesbehörden gewiß mehrere finden, welche die unter Reichsaufsicht stehenden kleinen Vereine an Bedeutung übertreffen.

\section{Gruppe V, Sonstige Versicherungszweige Glasversicherung}

Auf dem Gehiete der Glasversicherung berichtet die Statistik für das Jahr I9 IO über 21 deutsche Aktiengesellschaften, 1 deutschen Gegenseitigkeitsverein und 4 ausländische Gesellschaften. 8 Aktiengesellschaften, 1 Gegenseitigkeitsverein und 1 ausländische Gesellschaft beschäftigten sich ausschließlich mit diesem Versicherungszweige; für die übrigen Unternehmungen bildet die Glasversicherung nur einen Nebenbetrieb. Die Brasdenburger Spiegelglas-Versicherungs-Gesellschaft a. G. zu Brandenburg a. H. ist in eine Aktiengesellschaft umgewandelt worden und erscheint daher in der Statistik jetzt unter den Aktiengesellschaften.

Die Westdeutsche Versicherungs-Aktien-Bank in Essen hat die Glasversicherung in 1910 als neuen Geschäftszweig aufgenommen.

Der Rostocker Spiegelglas-Versicherungs-Verein auf Gegenseitigkeit zu Rostock ist durch Fusion auf die Mannheimer Versicherungsgesellschaft übergegangen.

Das Geschäft der Garantie Belge ist vom Jahre 191 I ab auf die Oberrheinische Versicherungs-Gesellschaft in Mannheim übergegangen und schon jetzt hier fortgelassen, weil dit Gesellschafi für das vorliegende Berichtsjahr keine ziffernmäßigen Angaben rechtzeiig gegeben hat.

De: Österreichische Phönix hat den deutschen Geschäftsbetrieb eingestellt.

Uber den Umfang des Geschäfts der deutschen Unternehmungen in der Glasversicherurg soll Tabelle V 1 unterrichten. Da die Versicherungssumme bei einer Gesellschaft nicht vollständig angegeben werden kann, so empfiehlt sich hier die Beschränkung auf diej’nigen Gesellschaften, welche für die Jahre I909 und 19 I0 alle Angaben haben

Die Unter. nehmungen machen können. Bei der Besprechung der Prämien und der Schäden im Anschluß an Tabelle V 4 wird Gelegenheit sein, auf den Geschäftsumfang aller Gesellschaften im Jahre I0 IO zurückzukommen.

An selbst abgeschlossenen Versicherungen bestanden einschließlich der in Rückdeckung gegebenen Beträge:

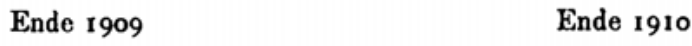

Dif durchschnittlich auf eine selbst abgeschlossene Versicherung entfallenden Beträge waren in Mark: 


\begin{tabular}{|c|c|c|c|c|c|c|c|c|c|}
\hline Ende des Jahres & 1902 & I 903 & 1904 & 1905 & 1906 & 1907 & I 908 & 1909 & I9 \\
\hline Aktiengesellschaften, Gesamtgeschäft......... & 489 & 492 & 504 & $5 \mathrm{I} 3$ & 525 & 537 & 543 & 549 & 552 \\
\hline inländisches Geschäft $\ldots \ldots \ldots \ldots \ldots \ldots$ & 491 & 494 & 504 & 514 & 532 & 537 & 546 & 548 & 547 \\
\hline ausländisches Geschäft ............. & $45 \mathrm{I}$ & 465 & 509 & 496 & 445 & 536 & 494 & 559 & 516 \\
\hline Gegenseitigkeitsvereine, Gesamtgeschäft ...... & $5^{19}$ & $5 \mathrm{r} 8$ & 529 & 533 & 544 & $55^{\circ}$ & $53^{8}$ & 535 & ${ }^{15}$ \\
\hline inländisches Geschãft $\ldots \ldots \ldots \ldots \ldots \ldots$ & 494 & 492 & 500 & 510 & 519 & $5^{26}$ & $5 \mathrm{II}$ & 506 & 518 \\
\hline ausländisches Geschäft $\ldots \ldots \ldots \ldots \ldots \ldots$ & 682 & 692 & 724 & 693 & 722 & 729 & 735 & 740 & 0 \\
\hline Insgesamt .... & 494 & 496 & 507 & $5^{16}$ & $5^{28}$ & 539 & 542 & 547 & \\
\hline
\end{tabular}

Außerdem haben die Aktiengesellschaften noch Versicherungen in Höhe von $79672 \mathscr{N}$ am Schlusse des Jahres 1909 und in Höhe von $116118 \mathscr{N}$ am Schlusse von 1910 als in Rückdeckung übernommen nachgewiesen. Der Anteil des mittelbaren Geschäfts am Gesamtgeschäft ist danach ganz unbedeutend gewesen; er stellte sich bei den Aktiengesellschaften im Jahre 1909 auf 0,03 und im Jahre I 9 IO auf 0,04 Prozent.

Von den selbst abgeschlossenenVersicherungen derjenigen Unternehmungen, bei denen die Zahl der Versicherungen und die Versicherungssummen angegeben sind, kommen:

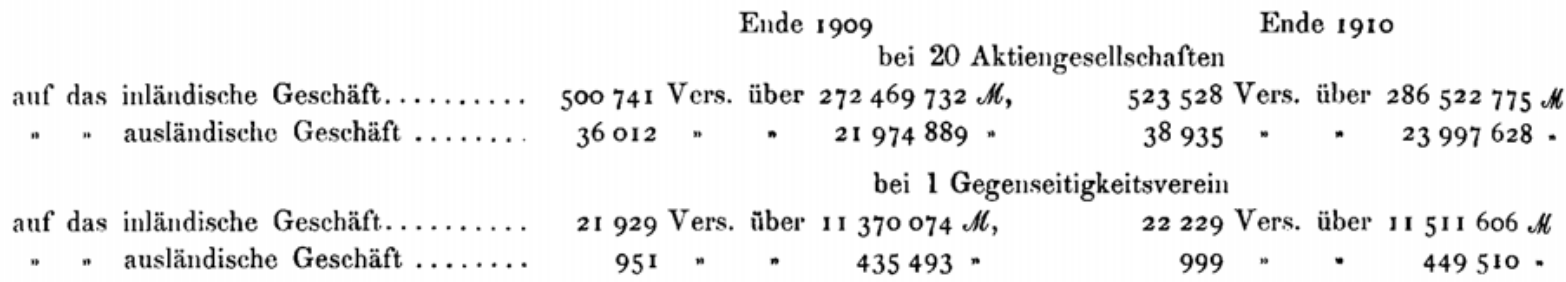

Diese Aktiengesellschaften hatten Ende 1909 noch $16396 \mathscr{M}$ im inländischen und $63276 \mathscr{N}$ im ausländischen Geschäft, Ende r 9 1 $045433 \mathscr{N}$ im inländischen und $70685 \mathscr{M}$ im ausländischen Geschäft in Rückdeckung übernommen.

Umfang dies deutsehen Geschäfts der ansländischen Unternehmungen

Die Bruttoprämien

und Schåden

Der Umfang des deutschen Geschäfts der ausländischen Gesellschaften ist nur klein (Tabelle V 3); das Geschäft liegt ganz überwiegend in der Hand einer Gesellschaft, die Ende r 9098326 und Ende r9 10 8374 Versicherungen hatte. Bei den übrigen 3 Gesellschaften waren Ende rgo9 1769 Versicherungen über $683165 \mathscr{M}$ und Ende r9io 1796 Versicherungen über $684579 \mathscr{M}$ in Kraft. Der durchschnittlich auf eine Versicherung kommende Betrag belief sich am Ende des Vorjahrs auf $386 \mathscr{M}$, am Ende des Berichtsjahrs auf $381 \mathscr{M}$. Das Geschäft ist von allen Gesellschaften nur unmittelbar betrieben worden.

Die Bruttoprämieneinnahme im Geschäftsjahr und die im Geschäftsjahr entstandenen Bruttoschäden sind für die deutschen Unternehmungen in Tabelle V 4 und für das deutsche Geschäft der ausländischen Gesellschaften in Tabelle V 5 zusammengestellt.

In der Glasversicherung haben im Jahre I 9 I 0 die 21 deutschen Aktiengesellschaften $8284925 \mathscr{N}$ und ein Gegenseitigkeitsverein $317159 \mathscr{M}$, alle Unternehmungen zusammen also $8602084 \mathscr{M}$ an Prämien eingenommen; den Aktiengesellschaften sind Schäden im Betrage von $5160977 \mathscr{M}$, dem Gegenseitigkeitsvereine solche im Betrage von $171101 \mathscr{N}$, zusammen im Betrage von $5332078 \mathscr{M}$ entstanden.

Der durchschnittliche Betrag eines Schadens stellt sich für die Aktiengesellschaften im inländischen Betriebe beim unmittelbaren Geschäft auf $48 \mathscr{N}$, im ausländischen Betrieb auf $25 \mathscr{M}$, für den deutschen Gegenseitigkeitsverein im Inland auf $42 \mathscr{M}$, im Ausland auf $59 \mathscr{N}$. 
Aus dem deutschen Glasversicherungsgeschäfte (Tabelle V 5) haben die 4 ausländischen Gesellschaften im Jahre 1910 insgesamt $172279 \mathscr{N}$ Bruttoprämien eingenommen; für Schäden hatten sie $121479 \mathscr{M}$ oder durchschnittlich für einen Schadenfall $61 \mathscr{M}$ bereitzustellen.

Die Ergebnisse des Geschäftsbetriebs sind bei der Glasversicherung, die von den Unternehmungen meist nur als Nebenbetrieb behandelt wird, in derselben Weise dargestellt wie bei der Feuerversicherung (S. $\left(2^{*}\right)$. Es ist in Tabelle V 6 der Gewinn des Berichtsjahrs allein aus dem Betriebe der Glasversicherung ermittelt, indem die Betriebs-Einnahmen den Betriebs-Ausgaben nebst Verwaltungskosten und Steuern gegenübergestellt sind. Der Gesamtgewinn der Unternehmungen ist in der Bilanz ausgewiesen (Tabelle V 8).

Die Betriebs-Einnahmen sind hier wie bei Gruppe IV die Prämien für eigene Rechnung, die Nebenleistungen der Versicherten, die Ersparnis aus der Schadenreserve des Vorjahrs, die Abnahme der Prämienüberträge und außerdem der Erlös aus Bruchglas; als Betriebs-Ausgaben wurden die Schäden des Geschäftsjahrs (nach Abzug des Anteils der Rückversicherer und der Schadenerstattungen), der Zuschuß zur Schadenreserve des Vorjahrs und die Zunahme der Prämienüberträge zusammengefaßt.

Bei den deutschen Unternehmungen betrug in der Glasversicherung:

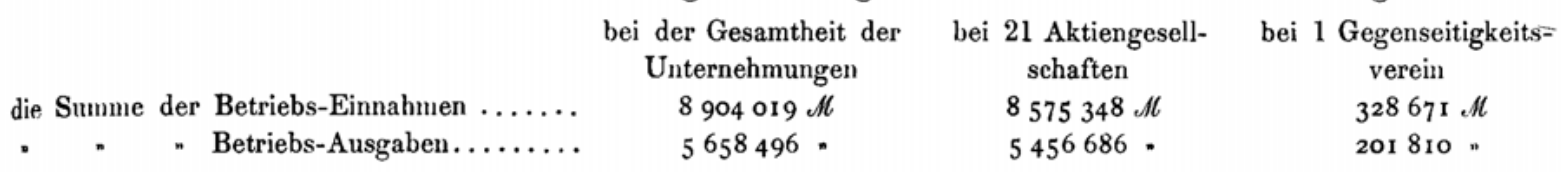

Von je $1000 \mathscr{N}$ der Betriebs-Einnahmen kamen:

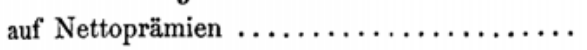

- Nebenleistungen der Versicherten......

- Ersparnis aus der Schadenreserve.....

$952 \mathscr{N}$

$19-$

15 .

- Abnahme der Prämienüberträge........

$(0,3)$ "

- Erlös aus Bruchglas.

14 .

$1000 \mathscr{M}$

n:

gaben kamen:

Von je $1000 \mathscr{M}$ der Betriebs-Ausgaben kan

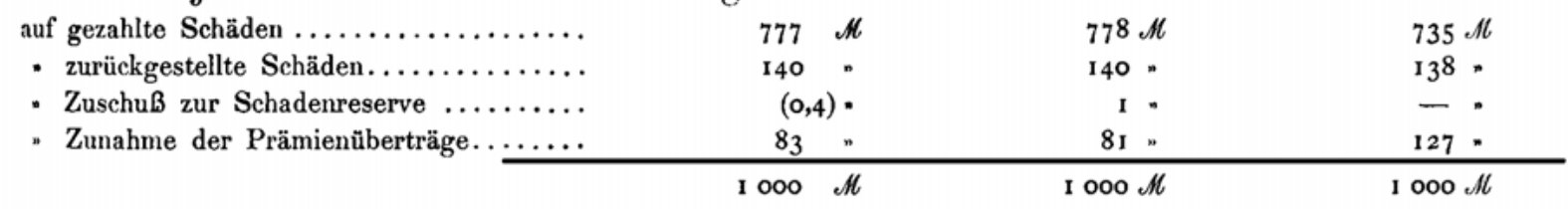

Von den im Geschäftsjahr entstandenen Schadensummen wurden durchschnittlich bei den Aktiengesellschaften 84,7 Prozent, bei dem Gegenseitigkeitsvereine 84,2 Prozent und bei allen Anstalten zusammen 84,7 Prozent noch im Geschäftsjahre gezahlt; der Rest wurde der Schadenreserve überwiesen.

An Verwaltungskosten und Steuern haben für die Glasversicherung die 21 Aktiengesellschaften $2625899 \mathscr{M}$ und der Gegenseitigkeitsverein $109762 \mathscr{N}$ aufgewandt. Aus dem reinen Versicherungsgeschäfte haben 4 Aktiengesellschaften einen Verlust von zusammen $15641 \mathscr{N}$ erlitten und die übrigen einen Gewinn von zusammen $508404 \mathscr{N}$ erzielt; der Gegenseitigkeitsverein hat einen Gewinn von $17099 \mathscr{M}$ gehabt.

Das Ergebnis der Vermögensverwaltung ist in Tabelle V 7 nur für diejenigen deutschen Unternehmungen zusammengestellt, deren Hauptgeschäft die Glasversicherung ist; das sind 8 Aktiengesellschaften und ein Gegenseitigkeitsverein.

BetricbsEinnabmen und Ausgalien und der Betriebsgewinn des Berichtsjahrs 
Unter den Erträgen stehen auch hier die Zinsen im Vordergrunde mit $358670 \varkappa$ bei den Aktiengesellschaften und mit $29047 \mathscr{M}$ bei dem Gegenseitigkeitsverein. Der reine Ertrag beläuft sich bei den Aktiengesellschaften auf $397444 \mathscr{M}$ und bei dem Gegenseitigkeitsverein auf $32368 \mathscr{M}$.

Die Bilanz Die Bilanz der deutschen Unternehmungen ist in Tabelle V 8 für dieselben $8 \mathrm{Ak}$ tiengesellschaften und einen Gegenseitigkeitsverein gegeben. Sie schließt für Ende I 9 Io ab mit $16070359 \mathscr{N}$ bei den Aktiengesellschaften und mit $815666 \mathscr{N}$ bei dem Gegenseitigkeitsverein. Im übrigen mögen hier folgende Zahlen zusammengestellt werden :

\section{Aktiva}

Verpflichtungen der Aktionäre oder Garanten.....

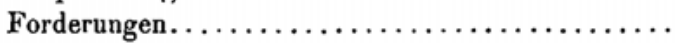

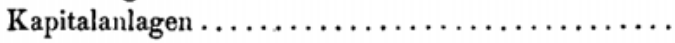

Kassenbestand $\ldots \ldots \ldots \ldots \ldots \ldots \ldots \ldots \ldots \ldots \ldots \ldots$

$$
\text { Passiva }
$$

Aktien- oder Garantiekapital. ..............

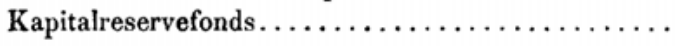

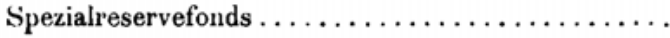

Guthaben anderer Versicherungsunternehmungen ..

Prämienüberträge $\ldots \ldots \ldots \ldots \ldots \ldots \ldots \ldots \ldots$

Schadenreserve.$\ldots \ldots \ldots \ldots \ldots \ldots \ldots \ldots \ldots$

$\begin{array}{rr}8 \text { Aktien- } & \begin{array}{c}\text { 1 Gegenseitig- } \\ \text { keitsverein }\end{array} \\ \text { gesellschaften } & -\quad M \\ 4085850 M & 47613 \text { " } \\ 2087215 " & 755988 " \\ 9644531 " & 12064 " \\ 196914 " & \end{array}$

\begin{tabular}{|c|c|c|c|}
\hline 5362000 & $\mathscr{M}$ & - & $\mathscr{M}$ \\
\hline 855859 & $n$ & 126769 & " \\
\hline 1351388 & $"$ & 12500 & $"$ \\
\hline 63530 & $"$ & - & $n$ \\
\hline 5389229 & $n$ & 396 I 96 & " \\
\hline I 558860 & $"$ & 64573 & " \\
\hline
\end{tabular}

Die Kapitalanlagen (Tabelle V 9) verteilen sich auf folgende Wertarten:

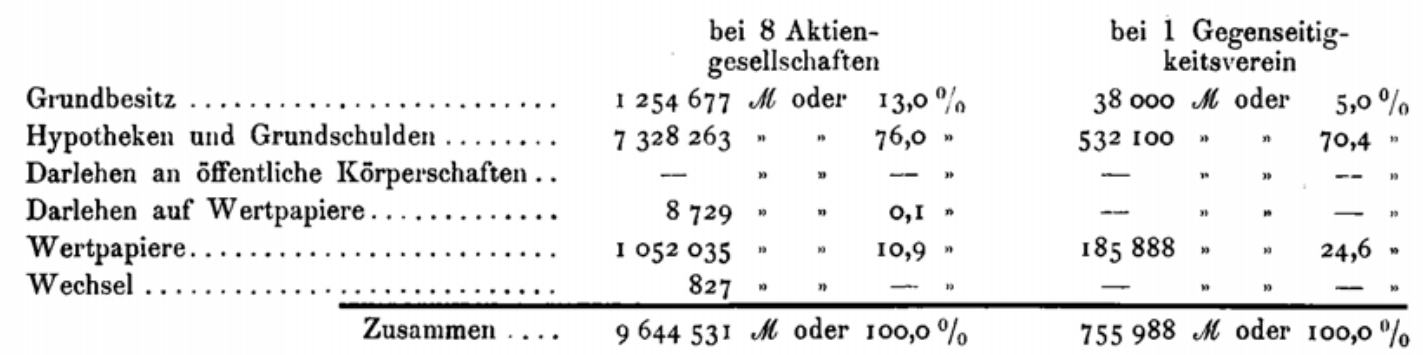

Die Kapitalanlagen sämtlicher 9 Unternehmungen haben Ende I910 insgesamt $884411 \mathscr{N}$ mehr betragen als am Schlusse des Vorjahrs. Zugenommen hat der Grundbesitz um $169474 \mathscr{N}$, der Hypothekenbestand um $1438149 \mathscr{M}$ und die Wertpapiere um $302394 \mathscr{N}$; dagegen sind die Darlehen auf Wertpapiere um $6624 \mathscr{N}$ und der Bestand an Wechseln um $870 \mathscr{N}$ zurückgegangen.

Der bilanzmäßige Gewinn ist nach Tabelle V 10 wie folgt verteilt worden:

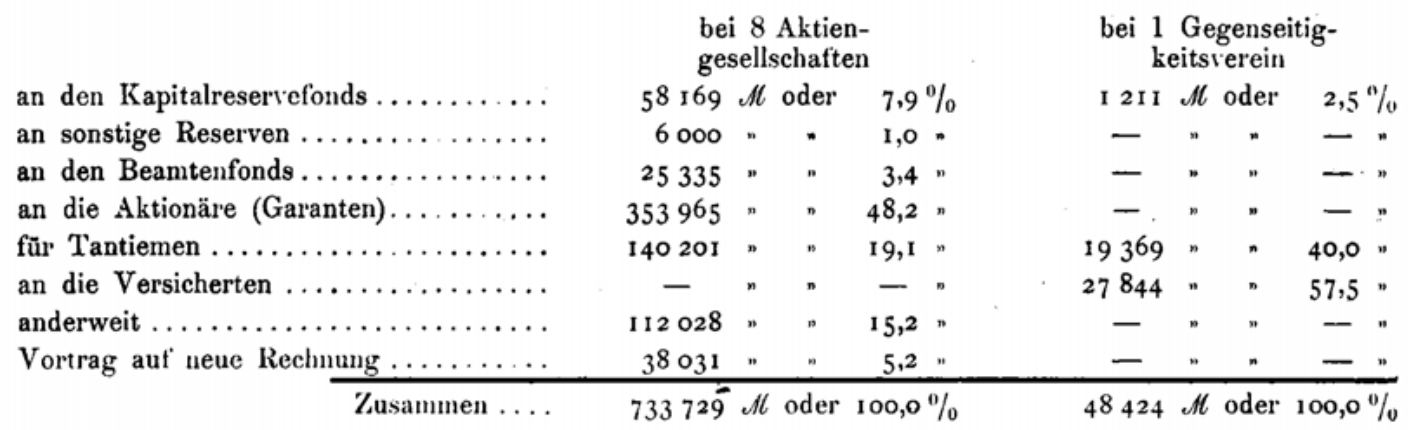


Der Kapitalreservefonds steht in der diesjährigen Bilanz bei den Aktiengesellschaften um $183552 \mathscr{N}$ höher und bei den Gegenseitigkeitsvereinen um $277345 \mathscr{N}$ niedriger als in der vorjährigen Bilanz. Die Spezialreserven sind bei den Aktiengesellschaften um $890 \$ 8 \mathscr{M}$ gewachsen, bei den (Gegenseitigkeitsvereinen um $113668 \mathscr{N}$ gesunken. Die Rückgänge bei den Gegenseitigkeitsvereinen haben ihre Ursache in dem oben erwähnten Ausscheiden zweier Vereine.

\section{Kautions- und Garantieversicherung}

Dieser Versicherungszweig wird nur nebenher betrieben. Auf das kleine Geschäft, das der Atlas in Ludwigshafen, die Wilhelma in Magdeburg und die Bayerische Versicherungs-Bank in München auf dem Gebiete der Kautionsversicherung erledigt haben, braucht hier nicht eingegangen zu werden (vgl. die Anmerkungen 4, 5 und 6 zu 'Tabelle I 30 S. 93). Von ausländischen Gesellschaften waren zum Betriebe dieses Zweiges im Deutschen Reiche lefugt die Schweizerische Unfall-Versicherungs-AktienGesellschaft in Winterthur und die Zürich; ihr Geschäft ist jedoch in den Tabellen nicht weiter berücksichtigt.

Uber den Umfang des Geschäfts der hier behandelten 3 Aktiengesellschaften und seine Zusammensetzung aus unmittelbaren und mittelbaren Abschlüssen sowie über den Anteil des inländischen Geschäfts am Gesamtgeschäfte gibt Tabelle V 12 Aufschluß. Danach liefen bei diesen Gesellschaften insgesamt an selbst abgeschlossenen Versicherungen einschließlich der in Rückdeckung gegebenen Beträge:

$$
\begin{array}{ccc}
\text { Ende } 1909 \ldots \ldots \ldots & 1427 & \text { Versicherungen über } 74786413 \mathscr{A} \\
n \quad 1910 \ldots \ldots \ldots & 1476
\end{array}
$$

so daß die Zahl der Versicherungen um 49 zugenommen hat, während die versicherten Summen um $7305345 \mathscr{M}$ zurückgegangen sind. Außerdem hatten zwei der drei Gesellschaften Ende 1910 noch $36015101 \mathscr{M}$ gegen $31527252 \mathscr{M}$ am Ende des Vorjahrs in Rückdeckung übernommen.

Von dem gesamten übernommenen Betrage von $103496169 \mathscr{M}$ liefen $55126894 \mathscr{N}$ für eigene Rechnung.

Von den selbst abgeschlossenen Versicherungen kamen:

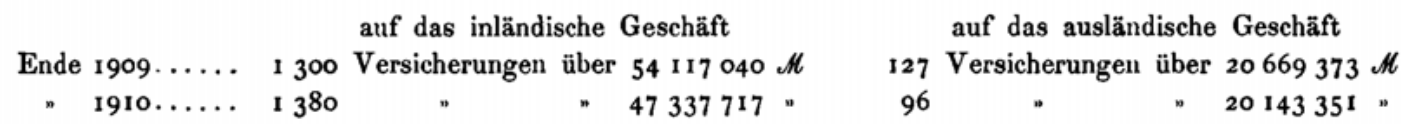

Die Bruttoprämieneinnahme hat nach Tabelle V 13 im Jahre $1910619104 \mathscr{M}$ betragen; davon kommen $514937 \mathscr{N}$ auf das inländische und $104167 \mathscr{A}$ auf das ausländische Geschäft. Von den überhaupt entstandenen Schadensummen in Höhe von $503464 \mathscr{M}$ kommen $259593 \mathscr{M}$ auf das Inland und $243871 \mathscr{N}$ auf das Ausland.

Die Betriebs-Einnahmen betrugen $510658 \mathcal{M}$, während die Betriebs-Ausgaben $427531 \mathscr{M}$ ausmachten; an Verwaltungskosten und Steuern sind $67829 \mathscr{M}$ aufgewandt worden. Eine Aktiengesellschaft hat aus dem Kautions- und Garantieversicherungsgeschäft einen Verlust von $67 \mathscr{M}$ gehabt; 2 Gesellschaften hatten einen Gewinn von zusammen $15365 \mathscr{M}$.

Die beiden ausländischen Gesellschaften hatten Ende 19 ro in Deutschland zusammen $13683125 \mathscr{M}$ versichert; sie haben im Berichtsjahre $159688 \mathscr{N}$ an Prämien eingenommen und $74585 \mathscr{M}$ für Schäden vergütet. 


\section{Kreditversicherung}

Die Kreditversicherung wird ebenfalls nur nebenher betrieben, und zwar nur vom Globus, Versicherungs-Aktien-Gesellschaft in Hamburg, und von einer ausländischen Gesellschaft. Es genügt hier, nur den Geschäftsumfang der deutschen Gesellschaft kurz zu skizzieren.

Vom direkten Geschäfte waren in Kraft:

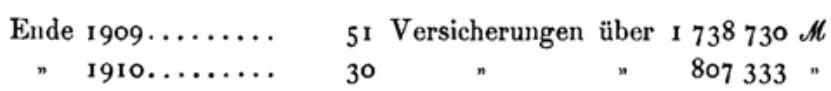

Es ist also die Zahl der Versicherungen um 21 und die versicherte Summe um 931397 . zurückgegangen. In Rückdeckung übernommen waren am Ende igiо $821300 \mathscr{M}$.

Ein erheblicher Teil der überhaupt übernommenen Summen wurde in Rückdeckung gegeben, von 1,63 Millionen Mark blieben 0,98 Millionen für eigene Rechnung.

Das direkte Geschäft stammt vollständig aus dem Deutschen Reiche.

Die Bruttoprämieneinnahme hat nach Tabelle V 13 im Jahre $191092896 \mathscr{N}$ betragen; davon kommen $57595 \mathscr{N}$ auf das inländische und $35301 \mathscr{N}$ auf das ausländische Geschäft. Von den Schäden in einem Gesamtbetrage von $70465 \mathscr{N}$ entfallen $50814 \mathscr{N}$ auf den inländischen und $19651 \mathscr{M}$ auf den ausländischen Betrieb.

Die Betriebs-Einnahmen betrugen $50886 \mathscr{M}$, während die Betriebs-Ausgaben $37970 \mathscr{M}$ ausmachten; für Verwaltungskosten und Steuern sind $23717 \mathscr{N}$ aufgewandt worden. Das Kreditversicherungsgeschäft für sich hat demnach einen Verlust von $10801 \mathscr{M}$ ergeben.

\section{Maschinenversicherung}

Die Maschinenversicherung ist im Jahre I9 IO von denselben 3 Gesellschaften wie im Vorjahre betrieben worden. Das Geschäft war noch fast ganz auf das Deutsche Reich beschränkt.

Nach Tabelle V 12 waren an selbst abgeschlossenen Versicherungen in Kraft:

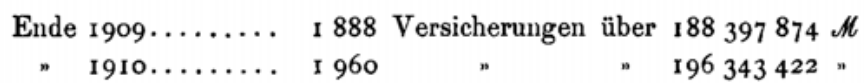

Im Berichtsjahre hat also die Zahl der Versicherungen um 72 und die Versicherungssumme um $7945548 \mathscr{N}$ zugenommen.

Von diesen Versicherungen kamen:

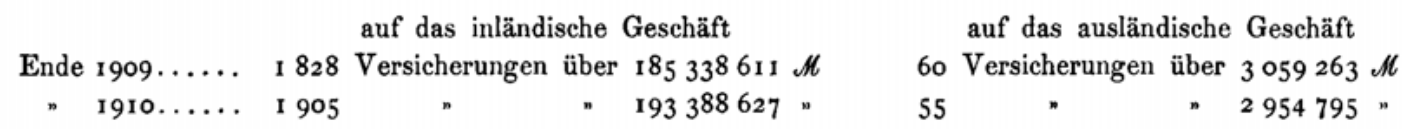

In der Maschinenversicherung läuft nur ein verhältnismäßig kleiner Teil für eigene Rechnung: von 196,34 Millionen Mark nur 38,72 Millionen.

Die Bruttoprämieneinnahme hat nach Tabelle V 13 im Jahre igio $417313 \mathscr{M}$ betragen; davon entfallen auf das inländisclie Geschäft $410100 \mathscr{M}$, auf das ausländische 
Geschäft $7213 \mathscr{N}$. Für Schäden sind $275072 \mathscr{M}$ gezahlt worden; davon betreffen $272727 \mathscr{N}$ inländische und $2345 \mathscr{N}$ ausländische Schadenfälle.

Die Betriebs-Einnahmen haben $120626 \mathscr{M}$ betragen, während die Betriebs-Ausgaben $65885 \mathscr{N}$ ausmachten; für Verwaltungskosten und Steuern sind $33838 \mathcal{M}$ aufgewandt worden. Das Maschinenversicherungsgeschäft hat einen Gewinn von insgesamt $20903 \mathscr{M}$ gebracht.

\section{Baulastversicherung}

Die Baulastversicherung ist im Jahre 1910 von demselben Gegenseitigkeitsvereine wie im Vorjahr, und zwar nur unmittelbar und nur innerhalb des Deutschen Reichs betrieben worden.

Nach Tabelle V 12 waren an selbst abgeschlossenen Versicherungen in Kraft:

$$
\begin{aligned}
& \text { Ende } 1909 \ldots \ldots \ldots \quad 202 \text { Versicherungen über } 4382800 \mathscr{A} \\
& \text { " } 1910 \ldots \ldots \ldots 200 \text { " " } 4522900 \text { " }
\end{aligned}
$$

Es hat also im Berichtsjahre die Zahl der Versicherungen um 2 abgenommen und die Versicherungssumme um $140100 \mathscr{N}$ zugenommen.

Von den übernommenen Versicherungen ist nichts in Rückdeckung gegeben worden.

Die Bruttoprämieneinnahme hat nach Tabelle V 13 im Jahre igio $19385 \mathscr{N}$ betragen; für Schäden sind $10403 \mathscr{N}$ gezahlt worden.

Die Betriebs-Einnahmen haben $19385 \mathscr{N}$ betragen; demgegenüber stehen die Betriebs-Ausgaben mit $11574 \mathscr{N}$ und die Verwaltungskosten und Steuern mit $1328 \mathscr{N}$, so daß ein Gewinn von $6483 \mathscr{M}$ zu verzeichnen ist.

\section{Wertgegenständeversicherung}

Diese Versicherungsart ist auch im Berichtsjahre nur von einer Aktiengesellschaft betrieben worden.

Es wurden nur unmittelbare Versicherungen (nur im Inlande) abgeschlossen. Das gesamte Geschäft umfaßte am Ende des Berichtsjahrs 160 Versicherungen über $2600619 \mathscr{N}$ gegenüber 72 Versicherungen über $1229821 \mathscr{N}$ am Ende des Vorjahrs. Die Prämieneinnahme belief sich auf $12579 \mathscr{N}$, für Schäden waren $4353 \mathscr{N}$ zu vergüten.

Die Betriebs-Einnahmen haben $8796 \mathscr{M}$ betragen, während die Betriebs-Ausgaben $4784 \mathscr{N}$ ausmachten; für Verwaltungskosten und Steuern sind $5420 \mathscr{M}$ aufgewandt worden. Das Wertgegenständeversicherungsgeschäft hat demnach einen Verlust von $1408 \mathscr{N}$ ergeben. 


\section{Veruntreuungsversicherung}

Die Veruntreuungsversicherung will für direkte Vermögensnachteile, die dem Versicherungsnehmer dadurch erwachsen, daß ein Angestellter in dienstlicher Eigenschaft eine Veruntreuung (Unterschlagung) von Geldern oder Wertpapieren zum Nachteile des Versicherungsnehmers begangen hat, Barersatz leisten. Dieser Versicherungszweig wurde auch im Berichtsjahre nur von einer Aktiengesellschaft und von dieser auch nur nebenher betrieben. Es wurden nur unmittelbare Versicherungen (nur im Inlande) abgeschlossen.

Nach Tabelle V 12 waren an selbst abgeschlossenen Versicherungen in Kraft:

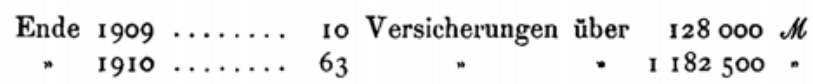

Es hat also im Berichtsjahre die Zahl der Versicherungen um 53 und die Versicherungssumme um $1054500 \mathscr{N}$ zugenommen. Von dem gesamten übernommenen Betrage von $1182500 \mathscr{M}$ liefen $236500 \mathscr{N}$ für eigene Rechnung.

Die Bruttoprämieneinnahme hat nach Tabelle V 13 im Jahre I9 Io $10940 \mathscr{N}$ betragen; für Schäden sind $5625 \mathscr{M}$ gezahlt worden.

Die Betriebs-Einnahmen betrugen $3826 \mathscr{N}$, während die Betriebs-Ausgaben $1476 \mathscr{N}$ ausmachten; an Verwaltungskosten und Steuern sind $4971 \mathscr{M}$ aufgewandt worden.

Das Veruntreuungsversicherungsgeschäft hat demnach einen Verlust von $2621 \mathcal{M}$ ergeben.

\section{Stellenlosenversicherung}

Die Stellenlosenversicherung wurde im Berichtsjahre unter Reichsaufsicht nur von zwei kaufmännischen Vereinigungen für ihre Mitglieder als Ergänzung ihrer Stellenvermittlung betrieben, und zwar ohne Rückversicherung.

Das Geschäft war bisher nicht eben belangreich: Ende r 909 waren 101812 und Ende I9 10 102637 Vereinsmitglieder versichert; im Laufe des Berichtsjahrs wurden an 1869 Mitglieder Entschädigungen gezahlt. Die Betriebs-Einnahmen wiesen für das Berichtsjahr $228402 \mathscr{M}$ Beiträge auf. Als Stellenlosenunterstützung wurden $105136 \mathscr{N}$ ausgezahlt und $8487 \mathscr{N}$ in Reserve gestellt. Die Verwaltungskosten betrugen $32569 \mathscr{M}$.

\section{Kaskoversicherung}

Die Kaskoversicherung wurde im Jahre 19 IO unter Reichsaufsicht von 36 deutschen und einem ausländischen Unternehmen betrieben. Von den deutschen Unternehmungen waren 33 Gegenseitigkeitsvereine und 3 eingetragene Genossenschaften. Der Internationale Bodensee-Motorsegelschiff-Verband in Arbon (Schwei\%) ist wegen seines geringen deutschen Geschäfts fortgelassen worden. 
Nach Tabelle V 15 waren versichert:

$\begin{array}{ccccc}\text { Ende } 1909 & \ldots \ldots \ldots & 8898 \text { Fahrzeuge mit } 90959112 \mathscr{M} \\ n \text { 1 } 910 & \ldots \ldots \ldots & 8923 & \text { " } 94688763 \text { " }\end{array}$

Im Berichtsjahre hat also die Zahl der versicherten Fahrzeuge um 25 und die Versicherungssumme um $3729651 \mathscr{M}$ zugenommen.

Die Summe der Einnahmen aller 36 Vereine hat im Jahre rgio $1619820 \mathscr{N}$ betragen, während die Summe der Ausgaben $1621882 \mathscr{M}$ ausmachte. 17 Vereine haben einen Uberschuß von $129518 \mathscr{M}$ erzielt; 16 Vereine haben einen Verlust von $131580 \mathscr{M}$ erlitten (Tabelle V 16).

Das Vermögen der Unternehmungen betrug Ende igo9 $4171969 \mathscr{M}$ und Ende ig10 $4441735 \mathscr{N}$; es hat also um $269766 \mathscr{N}$ zugenommen und war Ende igro angelegt zu 18,6 Prozent in Sparkasseneinlagen, zu 52,3 Prozent in Wertpapieren, zu 7,0 Prozent in Hypotheken, zu 1,7 Prozent in Kassenbestand und zu 20,4 Prozent in anderen Werten.

\section{Rückversicherung}

Von den deutschen privaten Versicherungsunternehmungen, welche die Rückversicherung in gesetzlich aufsichtspflichtigen Versicherungszweigen zum Gegenstande haben, sind für das Jahr 19 Io bei dieser Gruppe 25 behandelt, die in solchen Zweigen lediglich indirekt arbeiten, und außerdem noch 3, die in einigen Zweigen direkte und indirekte, in andern nur indirekte Geschäfte abschließen. Bei diesen letzteren Unternehmungen werden hier aber nur diejenigen aufsichtspflichtigen Zweige dargestellt, die nur indirekt betrieben werden; die übrigen sind bereits in den vorhergehenden Gruppen behandelt.

Sämtliche Unternehmungen sind Aktiengesellschaften.

Auf ausländische Versicherungsunternehmungen der in Rede stehenden Art bezieht sich die erwähnte Anordnung des Bundesrats nicht; sie unterstehen daher auch nicht der Aufsicht des Amts und kommen hier nicht in Betracht. 
Der Geschåtsumfang

Versicherungssummen, durch die sonst zumeist der Geschäftsumfang gemessen wird, sind die Gesellschaften nur bei der Lebensversicherung anzugeben in der Lage; bei den andern Zweigen können nur die Prämien und die Schäden herangezogen werden. Unter diesen Umständen scheint eine besondere Behandlung der Versicherungs. summen nicht angezeigt; es wird genügen, hier die Prämien und Schäden nach Versicherungszweigen zusammenzustellen (Tabelle V 19):

\begin{tabular}{|c|c|c|c|c|c|}
\hline \multirow{3}{*}{ Versicherungszweig } & \multirow{3}{*}{$\begin{array}{c}\text { Zahl der } \\
\text { Gesell- } \\
\text { schaften }\end{array}$} & \multicolumn{3}{|c|}{ Prämi en } & \multirow{3}{*}{$\begin{array}{c}\mathrm{Sch} \text { ä d en } \\
\text { für } \\
\text { eigene Rechnung } \\
\mathscr{M}\end{array}$} \\
\hline & & \multirow{2}{*}{$\begin{array}{c}\text { im ganzen } \\
\mathscr{N}\end{array}$} & \multicolumn{2}{|c|}{ für eigene Rechnung } & \\
\hline & & & $\mathscr{A}$ & $\%$ & \\
\hline Lebensversicherung $\ldots \ldots \ldots \ldots \ldots \ldots$. & I 4 & 59047801 & 41231271 & 698 & 12384669 \\
\hline Unfall- und Haftpflichtversicherung ..... & 14 & 27887652 & 24503428 & 879 & I0 900891 \\
\hline Transportversicherung.............. & 18 & 75355149 & 29942452 & 397 & 22464197 \\
\hline $\begin{array}{l}\text { Feuerversicherung (einschl. Mietverlustver- } \\
\text { sicherung usw.) } \ldots \ldots \ldots \ldots \ldots \ldots \ldots \ldots\end{array}$ & 26 & 235616767 & 131 318972 & 557 & 79751612 \\
\hline Hagelversicherung $\ldots \ldots \ldots \ldots \ldots \ldots$ & 2 & 6135412 & 6135412 & I 000 & 5740405 \\
\hline Sonstige Zweige $\ldots \ldots \ldots \ldots \ldots \ldots$ & 17 & Io 113945 & 9163429 & 906 & 3266217 \\
\hline Zusammen . & 28 & 414156726 & 242294964 & $\mathbf{5 8 5}$ & 134507991 \\
\hline In Jahre $1909 .$. & 28 & $35^{8} 013522$ & 214053036 & 598 & 122869978 \\
\hline
\end{tabular}

Die Gewinn- und Verlustrechnung (Tabelle V 18) bezieht sich auf den gesamten Geschäftsbetrieb der Unternehmungen; nur bei den bereits obenerwähnten drei Gesellschaften, die außer der Rückversicherung auch das direkte Geschäft in aufsichtspflichtigen Zweigen betreiben, sind hier nur diejenigen Zweige eingestellt, in denen sie lediglich mittelbar tätig sind. Wie sich das gesamte Geschäft aus den einzelnen Zweigen aufbaut, ist aus den bereits aus Tabelle V 19 mitgeteilten Zahlen zu ersehen. Hier bleibt nur noch folgendes zu erwähnen.

Die Vermögenserträge beliefen sich nach Abzug von $427663 \mathscr{M}$ Verlusten insgesamt auf $13051655 \mathscr{N}$; einem Kursgewinne von $171753 \mathscr{N}$ standen $427663 \mathscr{N}$ an Kursverlusten gegenüber. Von den Reserven haben nach Abzug der Abnahmen die Prämienreserven und Uberträge um $32815709 \mathscr{N}$, die Schadenreserven um $6632488 \mathscr{M}$ und die sonstigen Reserven um $6435913 \mathscr{M}$ zugenommen. Die Verwaltungskosten und Steuern haben $67731837 \mathscr{N}$ betragen.

Von allen 28 Gesellschaften hat nur eine einen Verlust von $143553 \mathscr{N}$ nachzuweisen gehabt; die übrigen haben einen Gewinn von zusammen $14430127 \mathscr{M}$ und mit Einbeziehung des Gewinnvortrags aus dem Vorjahre von 17890602 $\mathcal{N}$ erzielt.

Die Bilanzen der mehrfach erwähnten drei Gesellschaften, welche aufsichtspflichtige Versicherungszweige auch direkt betreiben, sind bereits bei Gruppe II, in Tabelle II 6 (Frankfurter Transport-, Unfall- und Glas-Versicherungs-Aktien-Gesellschaft und Oberrheinische Versicherungs-Gesellschaft), oder bei Gruppe IV, Tabelle IV 19 (Stuttgarter Mit- und Rück-Versicherungs-Aktien-Gesellschaft) nachgewiesen; es bleiben hier also nur noch die Bilanzen für 25 Gesellschaften zusammenzustellen, was in Tabelle V 20 geschehen ist. 
Aus dieser Tabelle sind folgende Zahlen zu entnehmen:

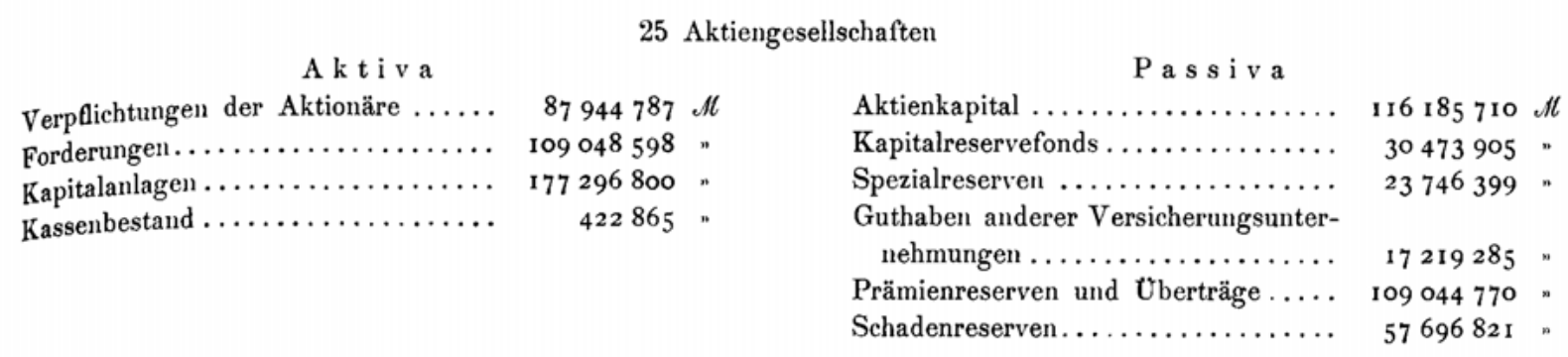

Die Kapitalanlagen (177296800 $\lll$ ) verteilen sich auf folgende Wertarten (Tabelle V 21): $4099810 \mathscr{M}$ oder 2,3 Prozent Grundbesitz, $44250025 \mathscr{N}$ oder 25,0 Prozent Hypotheken, $126366098 \mathscr{M}$ oder 71,3 Prozent Wertpapiere, $1115867 \mathscr{M}$ oder 0,6 Prozent Wechsel und $1465000 \mathscr{N}$ oder 0,8 Prozent Darlehen auf Wertpapiere.

Ubber die Wertpapiere mag folgendes mitgeteilt werden: Dem Nennwerte nach handelt es sich bei den betrachteten 25 Gesellschaften um insgesamt $153091179 \mathscr{M}$, wovon $85409975 \mathscr{N}$ oder 55,8 Prozent auf inländische und 67681204 $\mathcal{N}$ oder 44,2 Prozent auf ausländische Werte kommen. Die deutschen $\Lambda$ nlagen (55,8 Prozent der gesamten Anlagen) bestehen aus $51861750 \mathscr{M}$ (33,9 Prozent) Reichs- oder Staatsanleihen, $356000 \mathscr{N}(0,2$ Prozent) staatlich garantierten Anleihen, $7199900 \mathscr{H}(4,7$ Prozent) anderen öffentlichen Anleihen, $764 \check{5} 200 \mathscr{N}$ (5,0 Prozent) Pfandbriefen, $1104500 \mathscr{N}(0,7$ Prozent $)$ Obligationen und $806000 \mathscr{N}(0,5$ Prozent $)$ Aktien von Banken, Eisenbahn- und sonstigen industriellen Unternehmungen, $16436625 \mathscr{M}(10,8$ Prozent) Aktien von Versicherungsunternehmungen. Die ausländischen Anlagen (44,2 Prozent der gesamten Anlagen) bestehen aus $15828155 \mathscr{M}(10,3$ Prozent) Staats-, staatlich garantierten und andern öftentlichen Anleihen, $30905819 \mathscr{H}$ (20,2 Prozent) sonstigen Schuldverschreibungen und $20947230 \mathscr{N}$ (13,7 Prozent) Aktien.

Als Prämienreserven und Überträge sind, um die Übereinstimmung mit den Bilanzen der andern Gruppen zu wahren, auch hier nur die in eigener Verwahrung und Verwaltung der betrachteten Unternehmungen befindlichen Beträge in die Bilanz eingestellt worden. Tabelle V 22 zeigt, wie diese Beträge ermittelt wurden.

Die Bilanz weist nur bei einer Gesellschaft einen Verlust (von $139365 \mathscr{N}$ ) aus, der bilanzmäßige Gewinn der übrigen 24 Gesellschaften beträgt $17002076 \mathscr{N}$; er ist nach Tabelle V 23 wie folgt verteilt worden: an den Kapitalreservefonds $280415 \mathscr{M}$ oder 1,7 Prozent, an die sonstigen Reserven $4550781 \mathscr{M}$ oder 26,8 Prozent, an die Beamtenfonds $221535 \mathscr{M}$ oder 1,3 Prozent, an die Aktionäre $7077512 \mathscr{H}$ oder 41,7 Prozent, als Tantiemen $917476 \mathscr{M}$ oder 5,3 Prozent, als Vortrag auf neue Rechnung $3784377 \mathscr{M}$ oder 22,2 Prozent und endlich anderweit $169980 \mathscr{M}$ oder 1,0 Prozent. 



\section{Tafel}

zur

Versicherungs-Statistik des Aufsichtsamts für 1910 

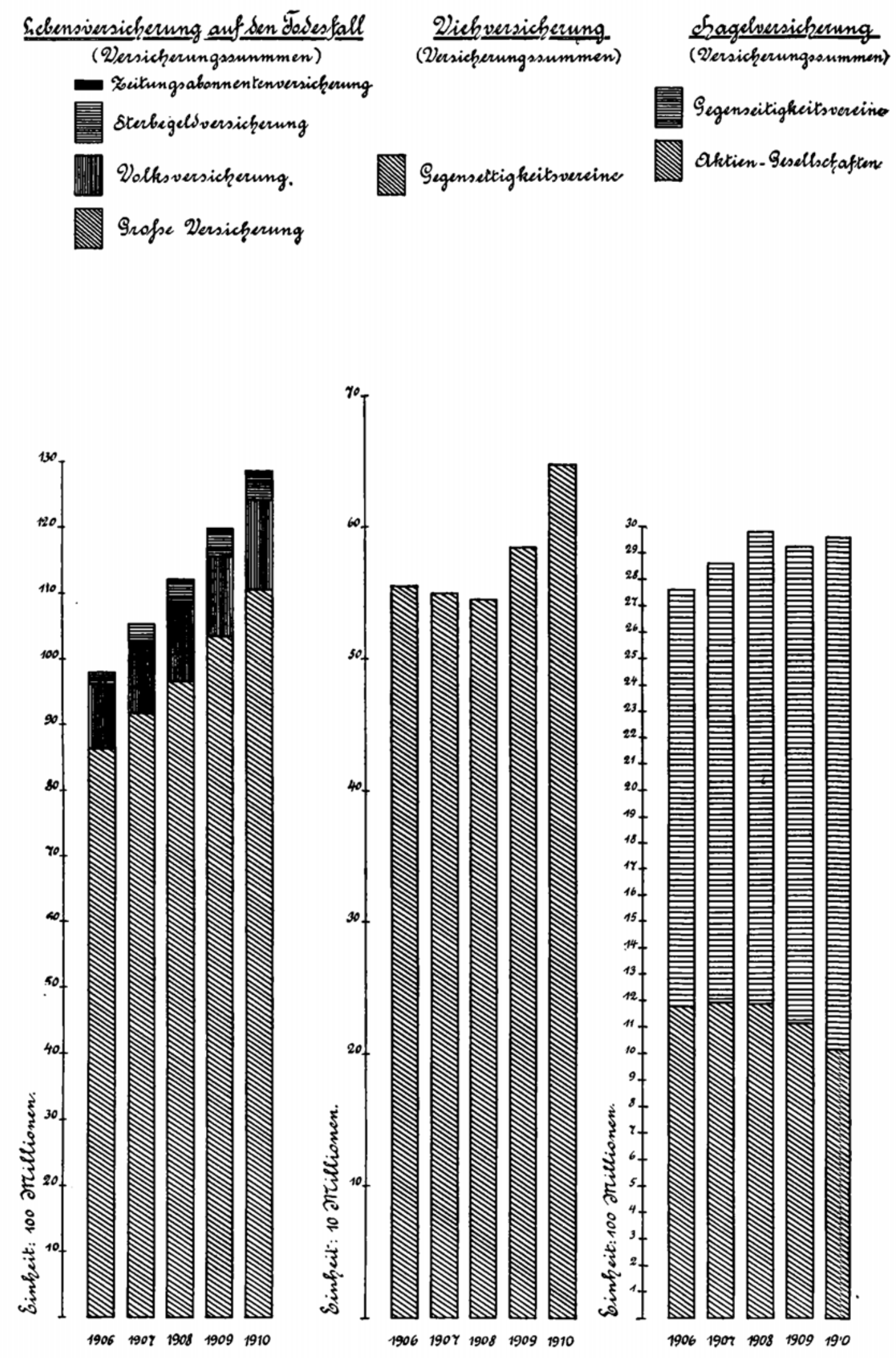

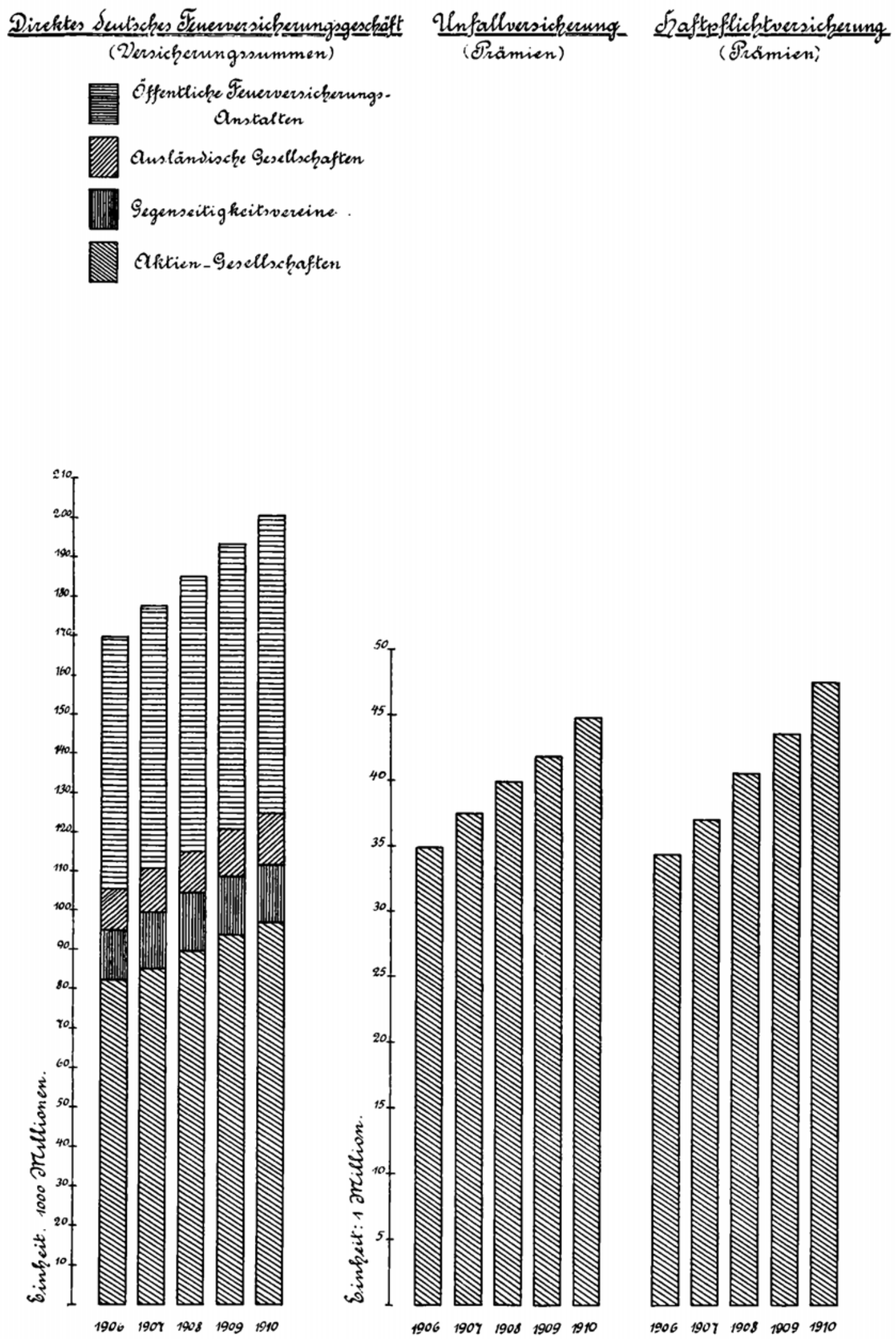

Versicherungs-Statistik 1910 
